University of Tennessee Health Science Center UTHSC Digital Commons

\title{
Novel Carbopol-Wax Blends for Controlled Release Oral Dosage Forms
}

Natarajansoundarapandian Mariageraldrajan

University of Tennessee Health Science Center

Follow this and additional works at: https://dc.uthsc.edu/dissertations

Part of the Pharmaceutics and Drug Design Commons

\section{Recommended Citation}

Mariageraldrajan, Natarajansoundarapandian , "Novel Carbopol-Wax Blends for Controlled Release Oral Dosage Forms" (2007). Theses and Dissertations (ETD). Paper 163. http://dx.doi.org/10.21007/ etd.cghs.2007.0201.

This Dissertation is brought to you for free and open access by the College of Graduate Health Sciences at UTHSC Digital Commons. It has been accepted for inclusion in Theses and Dissertations (ETD) by an authorized administrator of UTHSC Digital Commons. For more information, please contact jwelch30@uthsc.edu. 


\title{
Novel Carbopol-Wax Blends for Controlled Release Oral Dosage Forms
}

\begin{abstract}
Carbopol is crosslinked acrylic acid. Carbopol can be used in developing formulations for transdermal, oral, rectal use. It is forms strong gel in low concentration. Therefore, it can be used in low concentration in developing controlled release formulations. This increases the cost effectiveness and number of formulation options. In spite of its effectiveness, carbopol is one of the most efficient however underutilized polymer in oral controlled drug delivery system development. This is attributed to the difficulty in processing the carbopol. Carbopol has poor flow characteristics and stickiness. Objective of our research is to eliminate processing difficulties of carbopol using hot melt granulation process and to develop sustained release oral formulations of a basic (Propranolol $\mathrm{HCl}$ ) and an acidic drug (Glipizide).

Hot melt granulation was used to prepare free-flowing, directly compressible carbopol-wax blends. Evaluation of granular characteristics of carbopol-wax blends indicated that changes in the granular characteristics is dependent on process variables such as granulation temperature, granulation time and mixing speed. At higher granulation temperature, the granulation process became sensitive to granulation time and mixing speed. Thus, for developing robust granulation process to prepare carbopol-wax blends, the granulation must be done with lower granulation temperatures.

Carbopol is an acidic polymer and forms complex with basic drugs. Carbopol-basic drug complex has poor solubility. Hence oral controlled delivery system containing carbopol and basic drug, result in incomplete drug release. Water uptake and tablet erosion studies confirmed that incomplete drug release is a result of ionic complexation and absence of tablet erosion. Thus, by selecting appropriate carbopol grade, extent of ionic complexation was reduced and by using soluble filler in the formulation, an USP compliant controlled release oral formulation of propranolol hydrochloride was developed.

Controlled release properties of carbopol matrix containing Glipizide were evaluated. Formulations containing lactose as filler, either had long lag time or burst release based on the concentration of lactose in the formulation. Formulations containing Avicel yielded tablets with high hardness and zero order drug release. The drug release pattern of Avicel based formulations was dependent on drug-polymer ratio. Release of Glipizide from Avicel based formulations was dependent on compression force. Bioequivalent formulation of Glipzide was prepared using carbopol-wax blend.

Near infrared spectrophotometer was used to predict dissolution profiles of propranolol sustained release tablets non-destructively. Three different modeling algorithms were compared for their predictability. Knearest neighbors algorithm (KNN) yielded models with better predictability compared to partial least squares algorithm and support vector machines algorithm. Model validation was performed using independent data set. Model validation confirmed that KNN models can non-destructively predict dissolution profiles of sustained release propranolol tablets prepared at two different compression forces.
\end{abstract}

\section{Document Type}

Dissertation

Degree Name

Doctor of Philosophy (PhD)

Program

Pharmaceutical Sciences 
Research Advisor

Atul J. Shukla, Ph.D.

\section{Keywords}

carbopol, hot melt granulation, controlled release tablet, Nearinfrared, dissolution prediction

\section{Subject Categories}

Medicine and Health Sciences | Pharmaceutics and Drug Design | Pharmacy and Pharmaceutical Sciences 
NOVEL CARBOPOL-WAX BLENDS FOR CONTROLLED RELEASE ORAL DOSAGE FORMS

\author{
A Dissertation \\ Presented for \\ The Graduate Studies Council \\ The University of Tennessee \\ Health Science Center
}

\author{
In Partial Fulfillment \\ Of the Requirements for the Degree \\ Doctor of Philosophy \\ From The University of Tennessee
}

By

Natarajansoundarapandian Mariageraldrajan

May 2007 


\section{ACKNOWLEDGEMENTS}

I would like to thank my major professor, Dr. Atul J. Shukla for his valuable support and guidance. I would also like to thank my committee members, Dr. James R. Johnson, Dr. Bernd Meibohm, Dr. Casey Laizure, Dr. Yingxu Peng, Dr. Yichun Sun for their valuable suggestions and guidance.

I would also like to thank my present lab colleagues Dr. Wen Qu, Dr. Paras Jain, Om Anand, Chao Xiao, Namrata Trivedi, Sonia Bedi and my former lab colleagues Dr. Quanmin Chen, Dr. Bo Jiang, Dr. Shipeng Yu for their guidance and help. I thank my parents, wife and sister for supporting me at all times. My success has always been a fruit of their persistent support and help. I would like to thank Ms. Shirley Hancock for her kind helps and patience during format review of this dissertation.

Finally I would like to thank the College of Pharmacy at The University of Tennessee Health Science Center for giving me the opportunity to pursue my research. 


\begin{abstract}
Carbopol is crosslinked acrylic acid. Carbopol can be used in developing formulations for transdermal, oral, rectal use. It is forms strong gel in low concentration. Therefore, it can be used in low concentration in developing controlled release formulations. This increases the cost effectiveness and number of formulation options. In spite of its effectiveness, carbopol is one of the most efficient however underutilized polymer in oral controlled drug delivery system development. This is attributed to the difficulty in processing the carbopol. Carbopol has poor flow characteristics and stickiness. Objective of our research is to eliminate processing difficulties of carbopol using hot melt granulation process and to develop sustained release oral formulations of a basic (Propranolol $\mathrm{HCl}$ ) and an acidic drug (Glipizide).

Hot melt granulation was used to prepare free-flowing, directly compressible carbopolwax blends. Evaluation of granular characteristics of carbopol-wax blends indicated that changes in the granular characteristics is dependent on process variables such as granulation temperature, granulation time and mixing speed. At higher granulation temperature, the granulation process became sensitive to granulation time and mixing speed. Thus, for developing robust granulation process to prepare carbopol-wax blends, the granulation must be done with lower granulation temperatures.
\end{abstract}

Carbopol is an acidic polymer and forms complex with basic drugs. Carbopol-basic drug complex has poor solubility. Hence oral controlled delivery system containing carbopol and basic drug, result in incomplete drug release. Water uptake and tablet erosion studies confirmed that incomplete drug release is a result of ionic complexation and absence of tablet erosion. Thus, by selecting appropriate carbopol grade, extent of ionic complexation was reduced and by 
using soluble filler in the formulation, an USP compliant controlled release oral formulation of propranolol hydrochloride was developed.

Controlled release properties of carbopol matrix containing Glipizide were evaluated. Formulations containing lactose as filler, either had long lag time or burst release based on the concentration of lactose in the formulation. Formulations containing Avicel yielded tablets with high hardness and zero order drug release. The drug release pattern of Avicel based formulations was dependent on drug-polymer ratio. Release of Glipizide from Avicel based formulations was dependent on compression force. Bioequivalent formulation of Glipzide was prepared using carbopol-wax blend.

Near infrared spectrophotometer was used to predict dissolution profiles of propranolol sustained release tablets non-destructively. Three different modeling algorithms were compared for their predictability. K-nearest neighbors algorithm $(\mathrm{KNN})$ yielded models with better predictability compared to partial least squares algorithm and support vector machines algorithm. Model validation was performed using independent data set. Model validation confirmed that KNN models can non-destructively predict dissolution profiles of sustained release propranolol tablets prepared at two different compression forces. 


\section{TABLE OF CONTENTS}

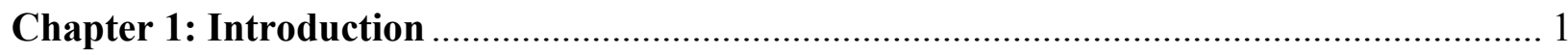

1.1. Types of oral controlled drug delivery systems ....................................................... 2

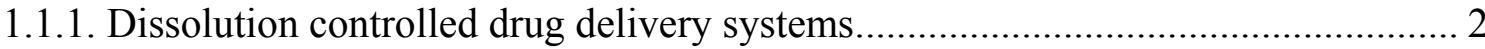

1.1.2. Diffusion controlled systems .................................................................. 3

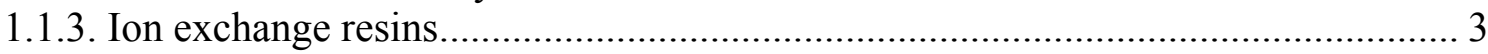

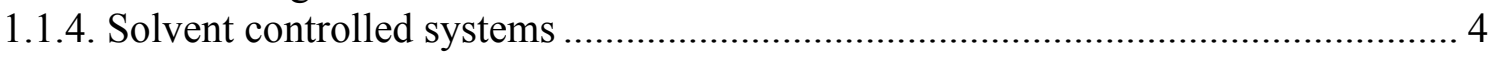

1.2. Hydrogel based oral controlled drug delivery systems ......................................... 5

1.2.1. Mechanism of drug release from hydrogels ................................................. 6

1.2.2. Empirical and semi-empirical mathematical models......................................... 9

1.3. Oral controlled drug delivery systems based on carbopol ....................................... 14

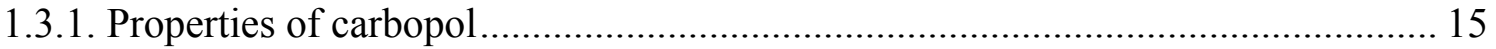

1.3.2. Drug release characteristics of carbopol matrix ......................................... 18

Chapter 2: Evaluation of Granular Characteristics of Carbopol-Wax Blend................... 36

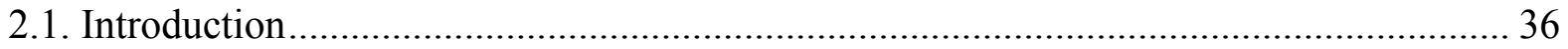

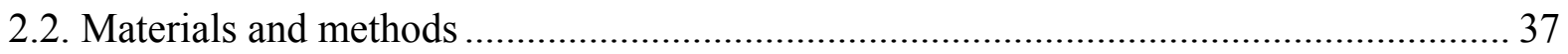

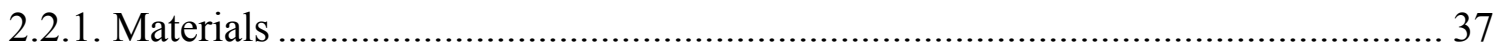

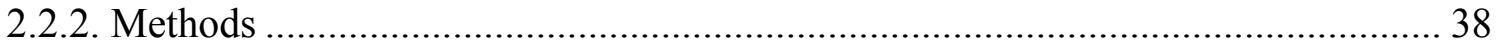

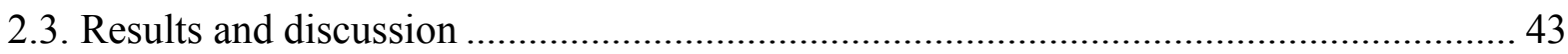

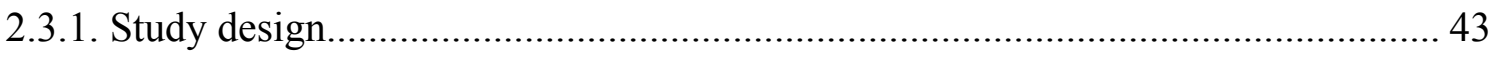

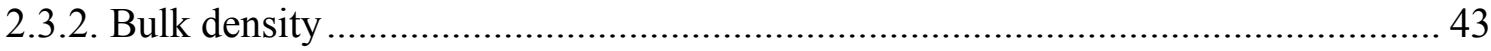

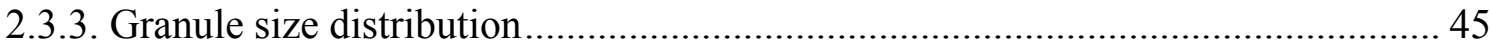

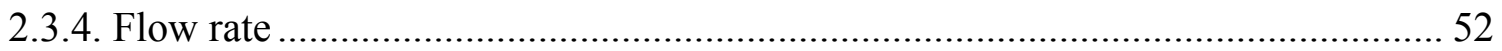

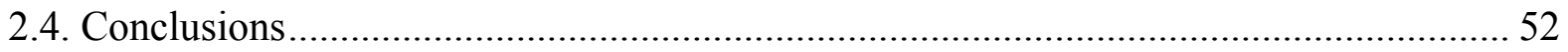

Chapter 3: Formulation Development of Propranolol Hydrochloride .............................. 55

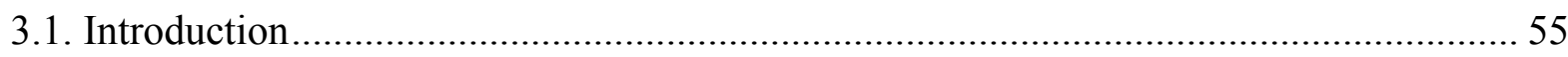

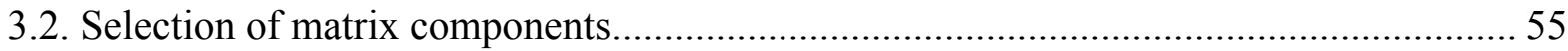

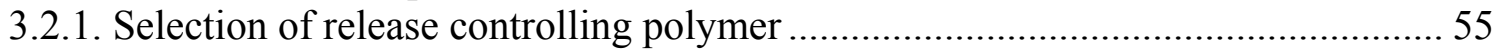

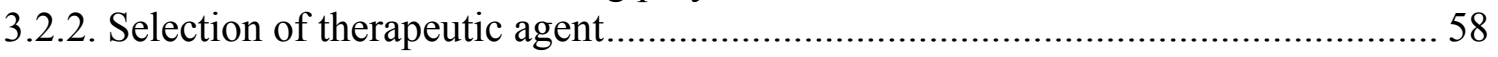

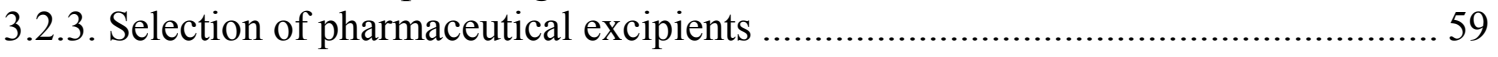

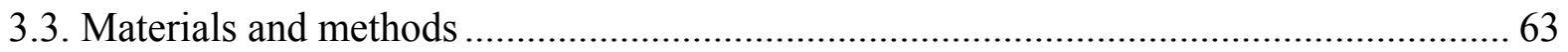




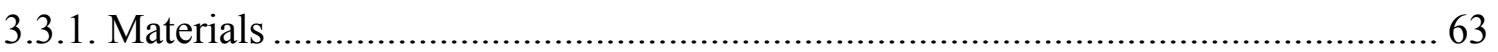

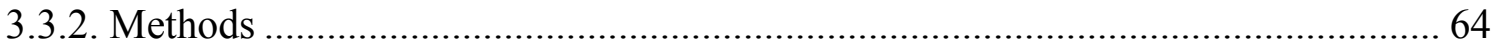

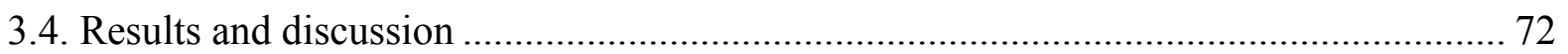

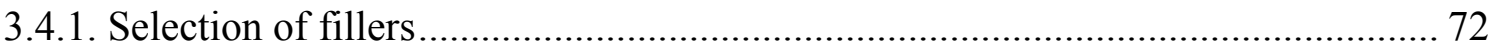

3.4.2. Development of controlled release formulation ................................................... 76

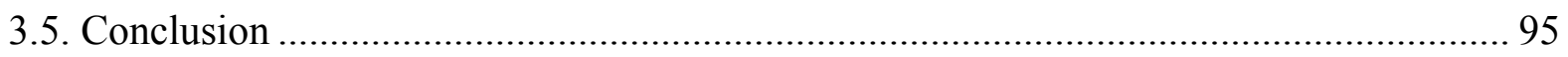

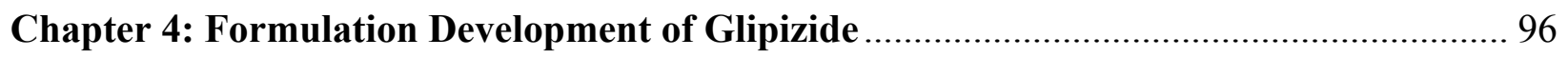

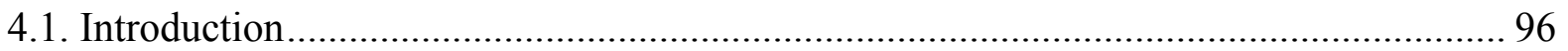

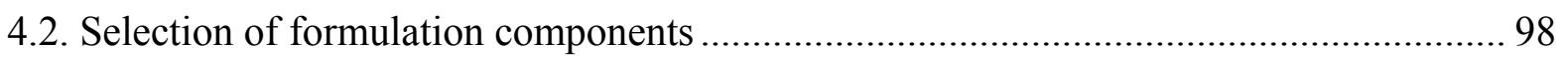

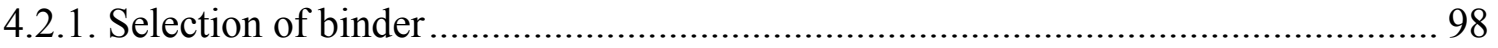

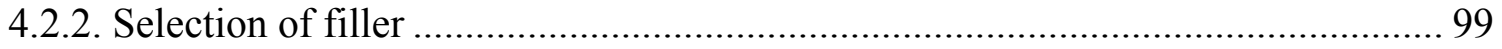

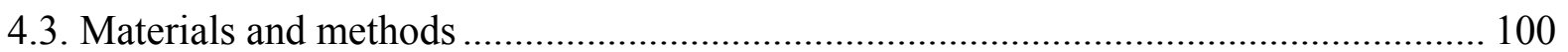

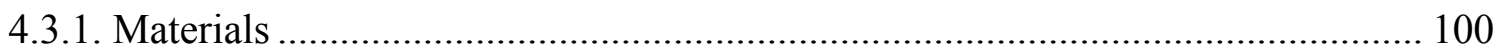

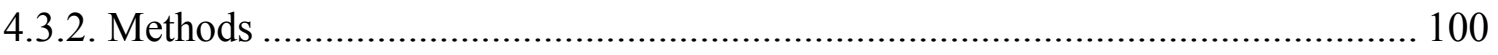

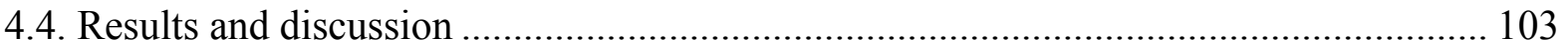

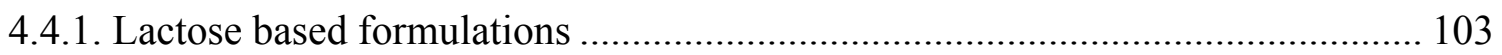

4.4.2. Microcrystalline cellulose based formulations ………....................................... 106

4.4.3. Formulation optimization .................................................................................. 109

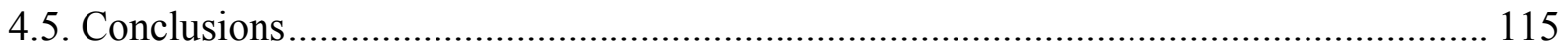

Chapter 5: Non Destructive Prediction of Dissolution Profiles Using NIRS....................... 119

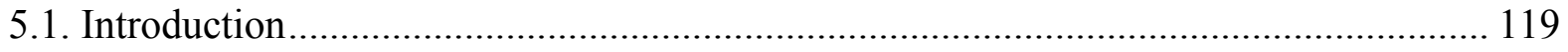

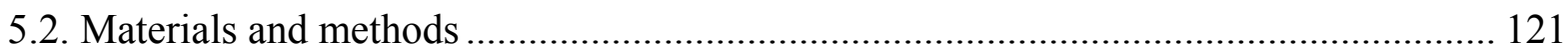

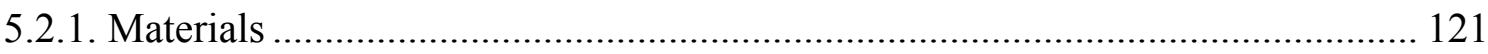

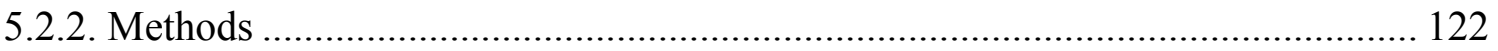

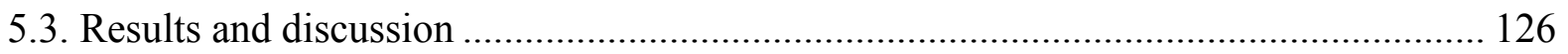

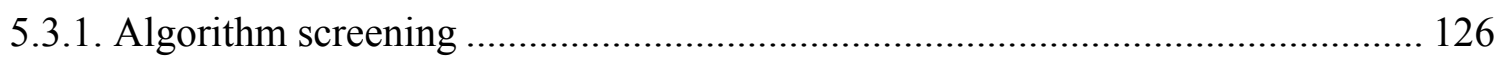

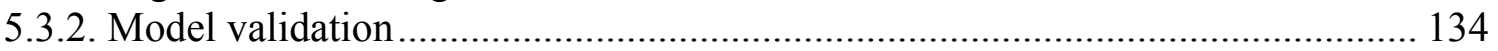

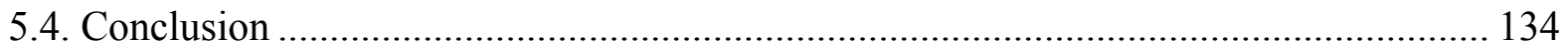

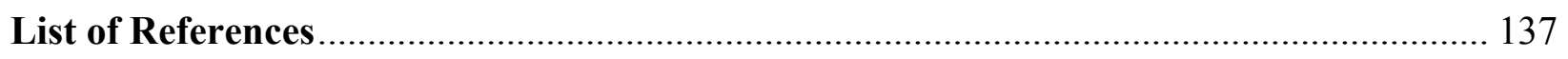

Vita 


\section{LIST OF TABLES}

Table 1.1. Release exponent values for devices of different geometry .................................. 13

Table 1.2. Releationship between carbopol grade and drug release mechanisms ..................... 25

Table 2.1. Process conditions used for preparing carbopol-wax blends .................................. 39

Table 2.2. Physical characteristics of carbopol-wax blends................................................... 41

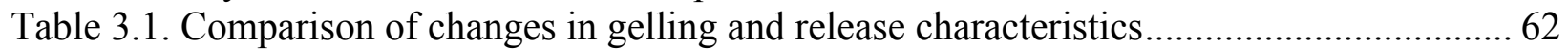

Table 3.2. Ingredients of formulations for selection of fillers ............................................ 65

Table 3.3. Composition of formulations for selection of fillers .......................................... 66

Table 3.4. Physical characteristics of formulations for filler selection................................... 68

Table 3.5. Statistical analysis of physical characteristics ................................................ 73

Table 3.6. Ingredients of formulations for product development ......................................... 77

Table 3.7. Composition of formulations for product development ........................................ 78

Table 3.8. Comparison between optimized formulation and USP specifications....................... 94

Table 4.1. Ingredients of formulations for glipizide product development ........................... 101

Table 4.2. Compositions of formulations for glipizide product development ......................... 102

Table 4.3. F2 values of formulations M 1 to M 4 ............................................................ 110

Table 4.4. F2 values of batches prepared using different compression forces ........................ 117

Table 5.1. Composition of formulations ........................................................................ 123 


\section{LIST OF FIGURES}

Figure 2.1. Effect of process conditions on bulk densities of formulations ............................. 44

Figure 2.2. Effect of process conditions on the amount of coarse granules ............................. 47

Figure 2.3. Effect of process conditions on the amount of medium size granules ..................... 49

Figure 2.4. Effect of process conditions on the amount of fine granules ................................ 51

Figure 2.5. Effect of process conditions on the dynamic flow rate of the granules.................... 53

Figure 3.1. Effect of carbopol on drug release.................................................................... 79

Figure 3.2. Effect of $10 \%$ sodium carbonate on dissolution profiles..................................... 80

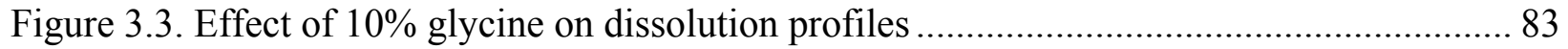

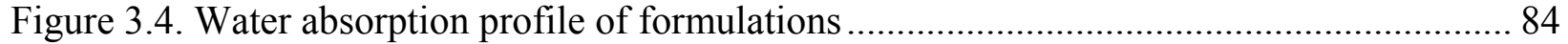

Figure 3.5. Erosion profiles of formulations.............................................................. 87

Figure 3.6. Relationship between water absorption and dissolution profiles ........................... 88

Figure 3.7. Relationship between erosion and dissolution profiles ........................................ 89

Figure 3.8. Effect of fillers on formulations containing carbopol 971P ................................ 91

Figure 3.9. Effect of carbopol grade on dissolution profiles ............................................ 93

Figure 4.1. Dissolution profiles of formulations containing lactose as filler........................... 104

Figure 4.2. Dissolution profiles of formulations containing MCC as filler............................. 108

Figure 4.3. Comparison of ANN predicted and actual dissolution profiles............................ 114

Figure 4.4. Effect of compression force on dissolution profiles............................................ 116

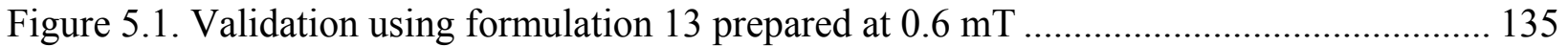

Figure 5.2. Validation using formulation 13 prepared at $1.2 \mathrm{mT}$........................................ 136 


\section{LIST OF ABBREVIATIONS}

ANN

Artificial Neural Network

GIT Gastro Intestinal Tract

Gms. Grams

GMS .Glyceryl Mono Stearate

$\mathrm{HCl}$ Hydro Chloric acid

KNN K-Nearest Neighbors

Kps. Kilopounds

MCC Micro Crystalline Cellulose

$\min$ minutes

$\mathrm{mT}$ metric Tons

NIR Near Infrared Spectrophotometer

NIRS Near Infrared Spectrophotometry

PDA Photo Diode Array

PLS. Partial Least Squares

RPM Revolutions Per Minute

$\mathrm{Sec}$ Seconds

SVM. Support Vector Machines

USP United States Pharmacopeia UV. .Ultra Violet 


\section{Chapter 1: Introduction}

"Patients don't take drugs, they take dosage forms".

"All drugs are toxins".

If we put above sentences together, we will realize that we give toxins to patients using dosage forms. Sixty years ago, although many scientists agreed that all drugs (toxins) are useful in curing diseases, not many scientists realized the dosage forms can offer distinct advantages such as reducing toxicity, extending therapeutic efficiency of drugs etc. However, now all pharmaceutical scientists and patients agree that pharmaceutical dosage forms make distinct difference in patient health care.

In last five decades, scientists have developed many types of delivery systems each with unique advantages. One of the oldest drug delivery systems is oral drug delivery systems. Oral drug delivery systems are popular far more than a century due to many distinct advantages such as ease of administration, palatability, ease of manufacturing, high patient compliance compared to parentral administration, no need for hospitalization etc.

Oral delivery systems include tablets, capsules, and molded pills. Since most of the drugs are available as solids and the stability of drugs is high at solid state, solid oral drug delivery systems are the most popular category of oral drug delivery systems among pharmaceutical scientists who explore use of different delivery systems for patient use. However, oral drug delivery systems are not "cure for all" technique. Modifications in the design of delivery system may be required based on needs. Needs may be different for each case.

One of the most profound needs is to maintain drug concentration in systemic circulation for longer period of time. This is one of the most desirable properties of an ideal drug delivery 
system. For, it reduces the frequency of administration and reduces the toxic effects of the drug. This objective can be achieved by formulating the drug in controlled release dosage form. Although there are multiple ways of designing delivering controlled release dosage forms, oral controlled drug delivery is one of the most popular and oldest techniques available. There are different types of oral controlled drug delivery systems available. They differ in the methods of manufacture, components of the system, method of administration etc.

One of the widely used classifications of oral controlled drug delivery systems involves use of mechanisms of drug release. This includes (i) dissolution controlled, (ii) diffusion controlled, (iii) ion exchange controlled, (iv) solvent controlled. Controlled release properties of oral drug delivery systems are imparted and optimized by selection of appropriate ingredients in the formulations. One of the most commonly used ingredients in formulation is a slowly dissolving gel forming polymer. Development and optimization of controlled drug release systems mandate understanding of gel forming polymers, their interactions with drugs and other matrix ingredients with different solubility, permeability, ionic characteristics etc. Some basic understanding of each type of oral controlled drug delivery system is discussed here:

\subsection{TYPES OF ORAL CONTROLLED DRUG DELIVERY SYSTEMS}

\subsubsection{Dissolution controlled drug delivery systems}

These types of systems employ dissolution control of active drug as release mechanism. This is one of the simplest types of systems. There are two types of dissolution controlled systems. They are (i) encapsulated products, (ii) matrix products. 
In the encapsulated products type, the active drug is coated with a slowly dissolving polymer or a wax. This coating controls the exposure of drug to the dissolution media. Since the availability of the dissolution media for dug dissolution is controlled, the release of the drug from coated particles is slow.

\subsubsection{Diffusion controlled systems}

Diffusion controlled systems employ control over drug diffusion from the device as opposed to the control over drug dissolution of dissolution controlled systems. Diffusion controlled systems are useful for drugs with poor solubility while dissolution controlled systems are useful for drugs with high solubility. There are two types of diffusion controlled systems. They are (i) reservoir devices, (ii) matrix devices. In reservoir devices, a drug core is surrounded by a polymeric membrane. In matrix devices, the active drug is dissolved or dispersed in polymeric matrix.

There are differences in mechanism of release kinetics followed by reservoir and matrix devices. While the drug release from reservoir devices can be explained by Fick's law, matrix devices follow Higuchi's equation.

\subsubsection{Ion exchange resins}

Unlike dissolution and diffusion controlled systems, the mechanism of drug release from ion exchange resins is different and depends on the exchange of ions between the dissolution media and the availability of exchange sites of the resins. First, the drug is complexed with the ion exchange resin, by exposing the saturated drug solution to calculated quantities of ion exchange resins. Then this product is either suspended or encapsulated in capsules for 
administration. Upon administration, the ions in the dissolution media (biological fluids in gastro intestinal tract) are exchanged. As a result, drug ions are replaced by the ions in the dissolution media. This replacement releases drug from ion exchange resins to biological fluids.

\subsubsection{Solvent controlled systems}

These systems employ control over solvent uptake of the system as opposed to controlling the dissolution or diffusion of the drug. Thus, these types of systems can be suitable for drugs wide differences in solubility. There are two mechanisms by which solvent controlled systems exercise control over the drug release. They are (i) osmosis, (ii) swelling.

Osmotic controlled systems have three basic components: (i) semi-permeable membrane, (ii) osmogen, (iii) delivery orifice. Semi-permeable is generally made up of cellulose acetate. It has selective permeability. The semi permeable membrane is designed in such a way that only water can permeate into the device and drug can not diffuse out of the device. The core of the device is coated with the semi permeable membrane. The core contains the drug and osmogen. Osmotic agents increase the osmotic pressure created, due to the dissolution of drug, inside the device. At high osmotic pressure, the contents of core are released through the delivery orifice. By optimizing the thickness of semi-permeable membrane, concentration of osmogen, diameter of the delivery orifice the drug release can be programmed precisely.

Next type of solvent controlled systems is swelling controlled system. Swelling controlled systems are one of the most extensively studied and utilized systems. This system consists of polymers that can swell in aqueous environment. Polymers that are used to prepare swelling controlled systems are generally called hydrogels. There are two types of hydrogels (i) water soluble, (ii) water insoluble. Both of these types absorb lot of (more than $100 \%$ of its 
original weight) water and form gel like structure in aqueous media. The viscosity and the resistance to drug permeability of these gels formed in aqueous media contribute to hydrogel's ability to control the drug release.

There is another classification of hydrogels based on its origin. This classification includes three types (i) natural, (ii) semi-synthetic, (iii) synthetic. Examples of each category are

i. Natural: gelatin, dextran, collagen, fibrin, chitosan, alginate.

ii. Semi - Synthetic: hydroxy propyl methyl cellulose, hydroxy ethyl cellulose, hydroxy propyl cellulose, ethyl cellulose.

iii. Synthetic: acrylic acid derivatives (Carbopol ${ }^{\circledR}$, Eudragits $\left.{ }^{\circledR}\right)$, hydroxy ethyl methacrylate, N-vinyl-2-pyrrolidone.

\subsection{HYDROGEL BASED ORAL CONTROLLED DRUG DELIVERY SYSTEMS}

As mentioned earlier, considerable attention is given to matrix controlled oral devices prepared using hydrogels because of their advantages. They are

i. Ease in processing. Systems containing swellable polymers have excellent compressibility and flowability characteristics. These two are prerequisites for routine manufacturing of tablet dosage forms.

ii. High drug loading. It is possible to develop systems with wide variety of drug loadings however with a significant control on the tablet size. This increases the formulation options.

iii. Design flexibility. Single or combination of polymers can be used to formulate the system. Polymers with different characteristics can be combined in a system to achieve unique advantages of the system. 


\subsubsection{Mechanism of drug release from hydrogels}

As mentioned earlier, hydrogels can absorb large quantities of water. So, their release mechanism is completely different from hydrophobic polymeric systems that release drug through pore diffusion process. In general, one of the following three modes of drug release is observed in hydrogel based formulations

i. Diffusion controlled.

ii. Swelling controlled.

iii. Chemically controlled.

Diffusion controlled systems can be explained using Fick's law with constant or variable diffusion coefficients.

Swelling controlled release from hydrogels is observed when the drug release is faster than hydrogel swelling. Typically, polymers used in these systems exist in glassy and rubbery states. These states of polymer define the boundaries in the delivery system. Modeling of drug release from these systems involves these boundary conditions. Two types of boundaries are observed in swelling controlled system. They are erosion boundary and swelling boundary. Erosion boundary is observed between the swollen gel layer and the dissolution media. Swelling boundary is observed between ungelled core (glassy polymer) and gelled layer (rubbery polymer). Distance between erosion boundary and swelling boundary is thickness of gelled layer. Ungelled layer of the tablet is called glassy core. The drug release depends on the thickness, viscosity of gelled layer and its interaction with drug molecules.

Chemically controlled release depends on chemical reaction within the matrix. For, the drug molecule is chemically attached to polymer chains. Thus, hydrolytic or enzymatic cleavage must set the drug free to be released. In some cases, surface or bulk erosion of polymers may aid 
drug release. Although this approach of chemically attaching drugs to polymer is popular research area, due to the regulatory restrictions it lacks market potential. Scientists have shown chemical cross linking can yield controlled release formulations that can release drug for multiple years with constant release rate. However, Food and Drug Administration considers an established drug as new molecule after any chemical modifications. This poses threat to very existence of the drug in the market and may need multi million dollar investments to get this product approved.

Understanding of drug release mechanisms from delivery system helps us to predict the extent of drug release based on the type of the system and polymers used to control the drug release. Modulation of drug release is possible only if the factors controlling the drug release are precisely understood. In an effort to understand the drug release mechanisms, many scientists have developed empirical and semi-empirical mathematical models explaining drug release. There are few processes associated with drug release from polymeric systems. As these processes determine the drug release, they are pivotal in understanding of the drug release characteristics of polymeric systems. These processes are (i) water uptake, (ii) polymer chain relaxation with volume expansion (swelling), (iii) drug diffusion, (iv) dissolution of polymer (erosion). In most cases, more than one of these processes takes place simultaneously. This creates a complex scenario. As these processes are time dependent, changes in the drug release can not be predicted accurately using a single mathematical model. However, closest approximations can be made using an appropriate model.

In general, diffusion, swelling and erosion are the important rate limiting mechanisms in most of the drug delivery systems. Diffusion can be explained using Fick's law. With onedimensional transport assumption, valid simple mathematical models can explain the drug 
release. This model is valid only for flat, planar devices. However, tablets are three dimensional powder compacts thus this simple model can not be used and may require complex mathematical models to explain/predict the drug release. Complexity in modeling such devices can be reduced by assuming constant diffusivity. However diffusivity is a function of water content of the system. Water content of the system changes with time invalidating the constant diffusivity assumption. Thus models based on constant diffusivity are less useful.

Polymer dissolution adds one more dimension to the problem that is already complex. Polymer dissolution will change the boundary conditions that are basically defined for explaining diffusion. Thus moving boundary conditions must be accounted in modeling such systems. Drug solubility may be another variable that can change the drug release mechanism. Drug solubility is an important factor when systems containing poorly soluble drugs are modeled.

In general, overall drug delivery process from swellable polymers e.g. HPMC, Carbopol can be described as follows.

i. Water concentration gradients are formed at polymer-water interface. This results in imbibition of the water into the matrix. This process depends on the physical status of the system. In dry systems the diffusion coefficient is low, where as in swollen gels it is similar to that of pure water. Water acts as a plasticizer and reduces the glass transition temperature of the polymer. Once the concentration of water is high enough to reduce the glass transition temperature to the temperature of the system, the polymer chains undergo transition from glass to rubbery state.

ii. Water imbibition causes polymer swelling and results in dimensional changes of the system. Geometric dimensions of the system increase as a result of swelling. 
iii. Contact of water with the drug in the system aid the dissolution of the drug and the dissolved drug diffuses out of the system.

iv. Continuous water penetration increases water content in the system. Increasing water content substantially increases the diffusion coefficient.

v. Poorly soluble drugs don't dissolve freely in the available water in the system. Thus, coexistence of dissolved and undissolved species of drug can be observed. However, non dissolved drug does not diffuse out.

vi. Systems containing high loading of freely soluble drugs undergo structural changes as drug diffuses out. As drug diffuses out the matrix becomes more porous and offers less resistance to the remaining drug in the matrix.

vii. Depending on the polymer type, extent of cross linking, degree of substitution the polymer dissolves slowly or rapidly. If the entire drug is released before any significant reduction in polymer content due to its dissolution, then this phenomenon is less important.

\subsubsection{Empirical and semi-empirical mathematical models}

\subsubsection{Higuchi equation}

In 1961, Higuchi published a mathematical equation to describe drug release from matrix systems [1]. Although it was valid for planar systems initially, it was later modified to include different geometries and matrix characteristics. Higuchi equation was derived under pseudosteady state assumptions and has less practical use when "real" controlled delivery system is modeled. The basic equation of Higuchi model is: 


$$
\mathrm{Mt} / \mathrm{A}=\sqrt{ } \mathrm{D}(2 \mathrm{Co}-\mathrm{Cs}) \mathrm{Cst}
$$

Where Mt is the cumulative absolute amount of drug released at time $\mathrm{t}, \mathrm{A}$ is the surface area of the controlled release device exposed to the release medium, $\mathrm{D}$ is the drug diffusivity in the polymer, Co is initial drug concentration and Cs is the solubility of the drug in the polymer. This equation can be written as:

$$
\mathrm{Mt} / \mathrm{M} \infty=\mathrm{K} \sqrt{\mathrm{t}}
$$

Where $\mathrm{M} \infty$ is the absolute cumulative amount of the drug released at the infinite time (equal to the drug loading in the system at time $\mathrm{t}=0$ ) and $\mathrm{K}$ is a constant for design variables of the system. Thus, drug release at any given time is proportional to the square root of the time. Most attractive feature of this Higuchi equation is its simplicity and applicability to many cases. However, we must be mindful of Higuchi equation when it is used. The assumptions are

i. Initial drug concentration in the system is much higher than the solubility of the drug. This assumption forms basis for pseudo-steady state approach.

ii. One dimensional diffusion forms basis of mathematical treatments used in the equation. Thus, equation is valid only if the edge effects must be minimal.

iii. Particle size of the suspended drug must be much smaller than the thickness of the system. iv. Swelling and/or polymer dissolution must be negligible.

v. Drug diffusivity remains constant throughout the drug release period.

vi. Perfect sink conditions are maintained. 
Evidently, these assumptions are invalid in most of the drug delivery systems. However, Higuchi equation can be used to get an abstract idea of underlying release mechanism. Simultaneous occurrence of many effects such as swelling, transition from glassy to rubbery state, polymer dissolution, water diffusion in and out of the system, drug diffusion out of the system may result in pseudo square root time kinetics. In general, proportionality between cumulative amount of drug released and square root of time is regarded as an indicator of diffusion controlled drug release.

\subsubsection{Power law}

Power law is a semi-empirical equation to describe the drug release from polymeric systems.

$$
\mathrm{Mt} / \mathrm{M} \infty=k t^{\mathrm{n}}
$$

Mt is cumulative amount of drug released at time t, M $\infty$ is cumulative amount of drug released at infinite time, $\mathrm{k}$ is release constant incorporating structural and geometric characteristics of the system, and $\mathrm{n}$ is the release exponent indicating the mechanism of drug release.

Peppas and co-workers $[2,3]$ not only introduced this equation but also explained uses and cautioned about its limitation. Power law is generalization of two independent drug release mechanisms. They are Fickian diffusion and Case II transport [4, 5]. In most systems containing swellable polymers, the drug release is additive result of polymer relaxation and diffusion [6]. Thus, power law gets one step closer to "reality" compared to Higuchi equation. 
Release exponent ' $n$ ' in the power law takes multiple values based on geometry of the system. By default the value of ' $n$ ' is 1 . This indicates that drug release is independent of time, in other words, zero order drug release or case-II transport. In this case, water imbibition is the rate limiting step in the drug release. As described earlier, water can act as plasticizer and change the physical state of the polymeric system from glassy to rubbery state. These transitions not only cause volumetric expansion of the system but also increase the mobility of the drug molecules.

When power law assumes 0.5 as the value of ' $n$ ' then it indicates diffusion controlled drug release, if the value of $n=1.0$ then it indicates swelling controlled drug release. If the values of ' $\mathrm{n}$ ' are between 0.5 to 1 , then it indicates combination of both diffusion and swelling mechanisms. This superimposition of both diffusion and swelling mechanisms is called anomalous transport. It must be remembered that these values are valid only for slab geometry. ' $\mathrm{n}$ ' values for spheres and cylinders $[7,8]$ are given in the Table 1.1.

\subsubsection{Other empirical and semi-empirical models}

Another mathematical model was developed by Peppas and Sahlin [9]. This is an extension of power law equation. It is

$$
\mathrm{Mt} / \mathrm{M} \infty=\mathrm{k}_{1} \mathrm{t}^{\mathrm{m}}+\mathrm{k}_{2} \mathrm{t}^{2 \mathrm{~m}}
$$

Where $\mathrm{k} 1, \mathrm{k} 2$ and $\mathrm{m}$ are constants. First term in the right hand of the equation represents the contribution of fickian diffusion (F) to overall drug release and the second term represents relaxational contribution (R). $\mathrm{R} / \mathrm{F}$ ratio can be calculated using following formula 
Table 1.1. Release exponent values for devices of different geometry

\begin{tabular}{cccc}
\hline & Release exponent $(\mathrm{n})$ & Release mechanism \\
\hline Slab & Cylinder & Sphere & \\
\cline { 1 - 3 } 0.5 & 0.45 & 0.43 & Fickian diffusion \\
$0.5<\mathrm{n}<1$ & $0.45<\mathrm{n}<0.89$ & $0.43<\mathrm{n}<0.85$ & Anomalous transport \\
1.0 & 0.89 & 0.85 & Case-II transport \\
\hline
\end{tabular}




$$
\mathrm{R} / \mathrm{F}=\mathrm{k}_{2} \mathrm{t}^{\mathrm{m}} / \mathrm{k}_{1}
$$

$\mathrm{R} / \mathrm{F}$ ratio can be used to identify the dominant release mechanism at any given time during the drug release. This can help to modulate the release profiles based on appropriate release mechanisms.

Thus far, this review of controlled drug delivery systems with emphasis on oral controlled drug delivery systems illustrated the mechanisms of drug release, materials used for preparation of swelling controlled oral controlled delivery systems. One of the most efficient however under utilized polymer that can be used to prepare oral controlled drug delivery systems is carbopol.

\subsection{ORAL CONTROLLED DRUG DELIVERY SYSTEMS BASED ON CARBOPOL}

Carbopol is polymers of acrylic acid crosslinked with allyl sucrose or allylpentaerythritol. Among multitude of polymers available in market, carbopol offers unique advantages when used in oral controlled release formulations [10]. They are

i. Systems containing carbopol as their major release controlling polymer show linear drug release (zero order release kinetics) for drugs with wide differences in solubility and doses.

ii. Carbopol is an excellent bioadhesive. Bioadhesivness of carbopol helps the drug delivery system by increasing the gastro intestinal transit time. Increase in gastro intestinal time improves the bioavailability and helps to achieve complete drug release from the dosage form.

iii. Carbopol forms a strong gel at low concentrations. High gel strength helps to achieve desired drug release profiles with low polymer concentration. Usage at low concentrations 
will increase cost benefits of delivery system, effective sustained release formulation can be designed with smaller size tablets and more formulation options will be available.

iv. Carbopol is synthetic polymer. Being synthetic, carbopol provides consistency in drug release profiles.

v. Carbopol has excellent compressibility. It forms hard tablets even with low compression forces.

As mentioned earlier, carbopol is one of the under utilized polymers in oral controlled drug delivery system development. Its usage is less compared to other cellulose polymers. However, there are studies [11-22] showing effectiveness of carbopol alone and in combination with other polymers to develop sustained release tablet formulations.

\subsubsection{Properties of carbopol}

\subsubsection{Physical and chemical properties of carbopol}

Carbopol is high molecular weight polymer. It is acrylic acid derivative prepared by chemical crosslinking with polyalkenyl alcohols or divinyl glycol. It is available as flocculated powders with average particle diameter of 0.2 micron. Surface static charge cause aggregation of submicron size particles. These particle aggregates can have particle size upto 2 to 7 microns. Essentially, each particle can be visualized as polymeric network containing polymer chains inter connected with multitude of cross links. Each polymer particle is capable forming strong gels when in contact with water and represents all properties of polymer. This unique property explains the efficiency of carbopol as controlled release polymer at significantly lower concentration compared to cellulose polymers. The polymer properties are controlled by degree 
of crosslinking which is dependent on amount of cross linker used during its preparation. Precise control over the extent of crosslinking adds consistency in the property of polymers unlike natural or semi - synthetic polymers. Cross linked polymers are insoluble in water however swellable in water. Most commonly used cellulose polymers such as Methocel ${ }^{\circledR}$ and Klucel ${ }^{\circledR}$ are uncrosslinked, linear polymers. They are water soluble and control drug release by swelling and erosion.

When in powder form with low water content ( 2 to $4 \%$ ), the glass transition temperature of Carbopol is $105^{\circ} \mathrm{C}$. However, the glass transition temperature drops dramatically when the polymer comes in contact with water as water is a good plasticizer for Carbopol. Plasticization of carbopol by water causes gyration of polymer chains. Radius of gyration continues to increase with time. Increase in radius of gyration, increases end-to-end distance of polymer chains, resulting in swelling of polymer. Carbopol can swell up to 1000 times its original volume and swelling is dependent on the $\mathrm{pH}$ of the aqueous media. Carbopol reaches its maximum swelling when $\mathrm{pH}$ of the environment is above its $\mathrm{pKa}$ of $6 \pm 0.5$.

\subsubsection{Molecular weight of carbopol}

It is impossible to measure accurate molecular weight of carbopol because of its extensive crosslinking. All analytical methods used for molecular weight determination require solubility of polymer as the measurements are made in dilute polymer solutions. Extensive crosslinking of the polymer chains make carbopol water insoluble hence impossible to estimate its molecular weight using conventional methods. However, using stoichiometric and theoretical calculations, molecular weight of carbopol can be estimated. It is calculated to be 3.5 billion. 
This high molecular weight is attributed to hundreds of polymer chains interconnected with cross links in a polymer molecule.

In linear polymers molecular weight and viscosity of the polymers are important factors controlling the drug release. However, in cross linked polymers like carbopol, they are unimportant. Nevertheless, the extent of crosslinking is important release controlling factor.

\subsubsection{Carbopol grades and their properties}

Introduced in mid 1960, carbopol is available in various grades. These are carbopol 934P, carbopol 974P NF, carbopol 971P NF and carbopol 71G NF. Carbopol 934P was one of the earliest grades of carbopol introduced to pharmaceutical market. However, benzene was used in crosslinking process in the preparation of 934P. Following toxicological concerns of residual benzene in carbopol 934P, pharmaceutical grades prepared with ethyl acetate were introduced. These are Carbopol 974P NF, carbopol 971P NF and carbopol 71G NF. Letter "P" in the name of carbopol grade indicates that this particular grade is suitable for pharmaceutical use. Letter "G" in the carbopol name indicates that this particular grade is available in granular form. Unless specified, all pharmaceutical grade carbopols are available as powders. All pharmaceutical grade carbopols have strong binding characteristics making them suitable for direct compression formulations. However, powder grades must be used in lowest necessary concentration in direct compression formulation as they have poor flowability. Nevertheless, granular grades have good flowability and can be used in high concentrations in direct compression formulations.

Carbopol 974P and $934 \mathrm{P}$ have similar rheological characteristics. In spite of their similar rheological characteristics, they show different release characteristics when used in the tablets at same concentrations. Carbopol 934P provides near zero order release profile through out the 
gastrointestinal tract, while carbopol 974P provides semi-enteric release profiles i.e., slow release in stomach and faster release in intestine. Analogically, based on their viscosity characteristics, carbopol 934P and carbopol 974P can be compared to mayonnaise and carbopol 971P can be compared to honey. Carbopol 971P is less crosslinked than 974 P. Carbopol 971P provides slow but more linear release than 974P. Carbopol $71 \mathrm{G}$ is granular form of 971P. Carbopol 71G has no chemical additives and prepared using roller compaction of carbopol 971P. Carbopol 71G has better flow characteristics than Carbopol 971P.

\subsubsection{Drug release characteristics of carbopol matrix}

\subsubsection{Mechanism of drug release from carbopol matrix}

Carbopol based matrix has stark contrast in drug release mechanism compared to matrix containing cellulose based polymers such as Hydroxy Propyl Methyl Cellulose (HPMC), Hydroxy Propyl Cellulose (HPC), Hydroxy Ethyl Cellulose (HEC) etc. Cellulose based polymers are water soluble linear polymers. They form gel layer on tablet surface upon hydration. Thickness, strength of the gel layer depends on molecular weight, polymer concentration and viscosity characteristics of gel layer. At higher polymer concentration, proximity of linear polymer chains increases probability of chain entanglement. Entangled polymer chains offer more resistance to drug release compared to individual polymer chains. Thus, drug release is inversely proportional to polymer concentration. Since cellulose based polymers are water soluble, swollen gel layer dissolves (erodes) away from the matrix. The extent of erosion depends on viscosity of the polymer which in turn depends on the molecular weight of the 
polymer. Thus, polymer with higher molecular weight releases drug slower compared to its low molecular weight counterpart.

Controlled release properties of carbopol marix are related to less extent with its molecular weight and viscosity and dependent on carbopol concentration in the matrix. Controlled release mechanism of carbopol matrix is dependent on extent of gellation and swelling. Extent of polymer swelling is dependent on degree of crosslinking and $\mathrm{pH}$ of the dissolution media. When in dry state, drug is entrapped in polymeric matrix in its glassy state. Upon contact with water, the glass transition temperature of polymer lowers below the external temperature turning the polymer from glassy state to rubbery state. In rubbery state, the mobility of polymer chains is high compared to that of polymer in its glassy state. However, chain entanglement due to increase in chain mobility is dependent on extent of cross linking. Carbopol, as described earlier, has individual polymer particles which are composed of polymer chains attached by crosslinks. Thus, each particle turns into gel in which the drug is dispersed. Crosslinks enhance ability of gel-particles to entrap the drug molecules.

Carbopol hydrogels are water insoluble unlike cellulose hydrogels. Thus, carbopol gels don't erode. However, they absorb lot of water and create osmotic pressure inside the gel. When fully hydrated, the osmotic pressure will break up the structure and shed off individual pieces of hydrogels. This unique property contributes carbopol's ability to release drug in near zero order fashion for long time [23-31]. It is hypothesized that upon gelling the surface of the polymer particles has gel layer. When glassy polymer turns into rubbery, hydrogel particles are formed. Each hydrogel particle can entrap the drug and form a drug reservoir. It can be imagined that hydrogel particle having drug is surrounded by swollen gel layer. Since the drug concentration is high in the hydrogel core, the thermodynamic activity of the drug increases and releases the drug 
from hydrogel. Swollen gel layer at surface of the hydrogel acts as rate controlling membrane thus helping to achieve linear drug release from hydrogels.

Since drug release from carbopol matrix depends on its hydrogel structure, factors influencing hydration and swelling of carbopol hydrogel can influence the drug release. Hydration and swelling of carbopol is dependent on crosslink density, extent of chain entanglement and crystallinity of the polymer. Rate of swelling is dependent on $\mathrm{pH}$ of the medium. Carbopol is an acidic polymer. It swells faster in alkaline $\mathrm{pH}$ compared to acidic $\mathrm{pH}$. Since each polymer particle is capable of forming hydrogel, the channels in the hydrogel is dependent on the concentration of polymer. Increase in polymer concentration will decrease the diameter of channels in the hydrogel and decrease the drug release. Thus, polymer concentration, extent of crosslinking, $\mathrm{pH}$ of the dissolution media can significantly influence the hydrogel properties hence drug release.

As mentioned earlier, thermodynamic potential of the drug is the chief driving force for the drug diffusion from carbopol hydrogel. Thermodynamic potential of the drug is a function of its solubility. Drugs with poor solubility will have poor thermodynamic potential and may partition into the hydrophobic domains of the hydrogels. Acrylic backbone of carbopol forms hydrophobic domain of the system. Thus, incase of poorly soluble drugs, drug partitioning may support carbopol's ability to control the drug release. Absence of matrix erosion provides stability for the carbopol matrix for longer period of time. Combination of these properties results in linear drug release profile of poorly soluble drugs from carbopol matrix. In contrast, cellulose based polymeric systems will show fickian release pattern with poorly soluble drugs. This is attributed to solubility of cellulose matrix. In case of highly water soluble drugs, fickian diffusion is observed with carbopol matrix. Although this behavior is similar to that of cellulose 
polymer, it is not due to the solubility of the matrix. It is attributed to quick dissolution of the drug or drug partition in to aqueous, low viscosity regions of the carbopol gels. Carbopol grades with high crosslinkinks e.g. Carbopol 974P offer large interstitial spaces where water is filled. Thus highly soluble drugs can quickly dissolve in these water filled channels and diffuse out of the matrix. In contrast, carbopol with less crosslinks e.g. Carbopol 971P form hydrogel with more uniform gel structure and less extent of water filled channels. Therefore, carbopol 971P is more effective than carbopol $974 \mathrm{P}$ in controlling drug release of freely soluble drugs. This is attributed to less number of interstitial channels and more uniform gel structure.

Compared to cellulose polymers, it is sometimes beneficial to use carbopol based systems for poorly soluble basic drugs. According to Henderson-hasselbach equation, the basic drugs have maximum solubility in acidic $\mathrm{pH}$ values. Thus, if cellulose polymers are used in formulating basic drugs, then acidic salts must be used in the formulation to solubilize the drug. Otherwise the drugs will precipitate in the tablet and will not be released to the fluids in GIT. However, carbopol is an acidic polymer. So, carbopol based sustained release matrix has acidic micro environment. Thus, it requires no acidic salt to maintain an environment to solubilize the drug.

Carbopol based tablets show limited erosion at high (more than 10\%) polymer concentration. This is attributed to entanglement of polymer chains resisting de-aggregation of polymeric matrix. Carbopol is water insoluble due to its extensive cross linking. Absence of matrix erosion and insolubility makes carbopol matrix very robust to wide differences in gastric motility, $\mathrm{pH}$ and fluid content in the gastro intestinal tract. Besides, tablets prepared using high compression forces are likely to have less interstitial space. This leads to reduction in number of channels for drug diffusion and result in decrease in drug release. 


\subsubsection{Crosslink density and drug release from carbopol matrix}

As discussed earlier, gel structure and drug characteristics are the most important factors that influence the drug release characteristics of carbopol matrix. Gel structure is dependent on the extent of crosslinking and differences in micro and macro viscosities of gel structure.

Upon hydration, carbopol 971P, grade with low crosslink density, forms "fishnet" gel structure. Low crosslinking permits polymer chains to open up as there is less constraints on the polymer structure. As the polymer opens freely upon hydration, the interstitial spaces are virtually eliminated and uniform gel is formed. This implies that there is less difference in the viscosity between micro and macro viscosity regions of the gel. This homogenous gel structure offers high resistance to drug diffusion compared to grades with high degree of crosslinking. Thus, carbopol 971P may be more efficient at lower concentration compared to carbopol $974 \mathrm{P}$ in controlling drug release.

Carbopol 974P, grade with high crosslink density, forms gel with "fuzzyball” structure. High number of cross links constrains the polymer swelling upon hydration. Therefore, the polymer does not open up leading to high difference in the viscosities of the different regions in the carbopol gel. These regions are called micro and macro viscosity regions based on their viscosity. Micro viscosity region has gel with low viscosity and macro viscosity region has gel with high viscosity. The differences in the gel viscosity stems from the differences in the water content in different regions of the gel. In carbopol 974P, since the polymer chains don't open up, they form gels with wide differences in viscosities of micro and macro viscosities.

Thus, in essence, carbopol gels are non-homogenous. They have micro and macro viscosity regions. Differences in the viscosity of micro and macro viscosity regions depend on the degree of crosslinking. Carbopol grades with high cross link density show higher differences 
in their viscosity between micro and macro viscosity regions while carbopol grades with low cross link density show lower difference in their viscosity between its micro and macro viscosity regions. The differences in the gel characteristics also imply that carbopol 974P forms rigid gel microparticles while carbopol 971P forms flexible gels when hydrated. Although carbopol 974P forms rigid gel particles, the drug release from carbopol 974P gels is higher compared to carbopol 934P and carbopol 971P. This is attributed to the higher number of channels present in their gel structure.

In simulated gastric fluid, carbopol gels are not fully hydrated. Based on their controlled release properties in the desending order, the carbopol grades can be arranged as carbopol 971P $>$ carbopol 934P $>$ carbopol 974P. In simulated intestinal fluid or in a media with $\mathrm{pH}>6$, the carbopol gels are fully hydrated. In fully hydrated states, based on their controlled release properties in the desending order, the carbopol grades can be arranged as carbopol 971P $>$ carbopol 934P $>$ carbopol 974P. The differences in the controlled release properties among different carbopol grades are attributed to their gel structure, differences in the number of channels in the gels formed etc. Carbopol 974P gels form rigid gel particles with more number of particles upon full hydration. Thus, when drug dissolves in the dissolution media, rigidity of the gel particles resist drug diffusion into the gel particles. This leads to accumulation of drug particles in interstitial spaces between gel particles. As these interstitial spaces turn into water filled channels over time, it results in high drug release. Carbopol 971P gels form flexible gels with less number of channels. Thus, when drug dissolves they can diffuse into gel particles. The uniform gel viscosity and less number channels resist the drug diffusion out of the gels. Therefore, drug diffusion into the gel structure is the rate controlling factor in drug release from carbopol gels and it is dependent on extent of crosslinking. 


\subsubsection{Effect of drug solubility on drug release from carbopol matrix}

Mostly carbopol polymer shows extended release profiles when fully hydrated when loaded with drugs with moderate water solubility i.e., less than $100 \mathrm{mg} / \mathrm{ml}$ ). Nature of drug release profiles is dependent on the degree of crosslinking, water solubility of the drugs and $\mathrm{pH}$ of the dissolution medium. Table 1.2 summarizes the effect of these factors on the pattern of drug release

In general, water soluble drugs exhibit square root time release kinetics in both simulated gastric fluid and simulated intestinal fluid in all three grades of carbopol. However, poorly water soluble drugs exhibit near zero order release profiles in carbopol 934P and carbopol 971P and they show semi-enteric (less amount of drug released in acid and more amount of drug released in phosphate buffer) profile in carbopol 974P.

Highly water soluble drugs like norephedrine $\mathrm{HCl}$ [32] from carbopol matrix are released through partition. Thus, drug dissolution is the release controlling mechanisms in such cases. Once dissolved the drug partitions into low viscosity region in the microstructure and released through channels.

Perez-Marcos et al $[30,31]$ compared the release mechanisms of carbopol matrix for drugs with different solubility. Highly water soluble drug, atenolol, exhibited square-root-of-time dependent drug release while poorly water soluble drug, furosemide, exhibited zero order release. Huang et al [33] studied the effect of drug solubility on carbopol 934P matrix. Theophylline, a poorly soluble drug, exhibited fickian diffusion in SGF. Water soluble drugs, such as chlorpheniramine maleate and sodium salicylate, exhibited fickian diffusion in SGF. These studies confirm the significant relationship between drug solubility and drug release pattern from carbopol based matrix systems. 
Table 1.2. Relationship between carbopol grade and drug release mechanisms

\begin{tabular}{ccccc}
\hline \multirow{2}{*}{ Carbopol grade } & \multicolumn{2}{c}{ Highly water soluble drugs } & \multicolumn{2}{c}{ Poorly water soluble drugs } \\
\cline { 2 - 5 } & $\mathrm{pH}=1.2$ & $\mathrm{pH}=7.5$ & $\mathrm{pH}=1.2$ & $\mathrm{pH}=7.5$ \\
\hline 934P NF & Fickian & Fickian & $\begin{array}{c}\text { Non-fickian/ } \\
\text { Anomalous }\end{array}$ & $\begin{array}{c}\text { Anomalous/ } \\
\text { Case II }\end{array}$ \\
971P NF & Fickian & Fickian & $\begin{array}{c}\text { Non-fickian/ } \\
\text { Anomalous }\end{array}$ & $\begin{array}{c}\text { Anomalous/ } \\
\text { Case II }\end{array}$ \\
974P & Fickian & Fickian & $\begin{array}{c}\text { Non-fickian/ } \\
\text { Anomalous }\end{array}$ & Fickian \\
\hline
\end{tabular}


It is postulated that if the barrier controlled mechanism is predominant than diffusion, then a zero order drug release pattern is observed in carbopol matrix. In cases where zero order or near zero order release is observed, hydrated polymer may act as physical barrier for drug diffusion or polymer relaxation could slower the drug release.

\subsubsection{Effect of $\mathrm{pH}$ on drug release}

Carbopol is weakly acidic polymer. The maximum swelling of the polymer is achieved in basic $\mathrm{pH}$. Oral controlled release formulations first reside in stomach before reaching intestine where the $\mathrm{pH}$ is basic. Acidic stomach $\mathrm{pH}$ does not support carbopol gelling. Thus with most drugs fickian release pattern is observed in stomach $\mathrm{pH}$. Basic intestinal $\mathrm{pH}$ supports complete swelling of carbopol as it causes ionization of carboxylic acid groups. Ionization of carboxylic acid groups in polymer chains result in repulsion of polymer chains which is manifested as polymer swelling.

Upon complete hydration and swelling, carbopol polymers exhibit a special type of nonfickian anomalous release with poorly soluble drugs. It is called case II or near zero order drug release. It is attributed to formation of gel barrier and gel micro particles in carbopol based matrix systems. This is called barrier controlled release mechanism. Upon hydration, gel micro particles trap the drug inside the particles. Surface gel layer act as barrier coating controlling the drug release. As the drug particles are solubilized, their thermodynamic potential migrate the solubilized drug to the surface gel barrier. Now, Surface gel barrier contains saturated drug solution and maintain the concentration gradient required for uniform drug release. Drug diffuses out from saturated gel barrier to channels in the micro viscosity regions of the carbopol matrix. 
Since each gel micro particle can serve as drug reservoir, this process of uniform drug release adds up and results in zero order drug release from the matrix.

F. Bulut-Oner et al [34] used isoniazid, a water soluble drug, with 30\% carbopol 934P matrix to study the effect of $\mathrm{pH}$ on the drug release characteristics. While all drug was released in less than fifty minutes in SGF, the drug release was sustained for seven hours in SIF and sustained for five hours in distilled water. Thus, at neutral and near neutral $\mathrm{pH}$ boosts the efficiency of carbopol matrix to sustain the drug release.

Study by A.M. Cooper et al [32] illustrate the effect of $\mathrm{pH}$ on the drug release with carbopol matrix is not without exceptions. Testing basic CNS compounds in HPMC and Carbopol 974P matrices, authors confirmed similar fickian diffusion type release profiles with carbopol matrix in both SGF and SIF. This implies that nature of drug plays critical role in drug release characteristics of carbopol matrix. Thus, whenever the effect of $\mathrm{pH}$ on the drug release from carbopol matrix is discussed, it must be done in context to the nature of drug.

Another special case is carbopol matrix containing ionizable drugs. Carbopol is an anionic polymer. In basic $\mathrm{pH}$, carboxylic acid groups of carbopol ionize and the gel fully hydrates. Basic drugs undergo complexation with anionic carbopol when both drug and carbopol are ionized. In most cases, the drug - carbopol complex has less solubility than the pure drug. This supports carbopol's sustained release properties. Therefore, $\mathrm{pH}$ independent, zero order release profiles are observed for basic compounds in carbopol matrix. Carbopol has carboxylic groups to the extent of $62 \%$. This makes basic drugs most vulnerable for complexation $[35,36]$. Complexation not only provides $\mathrm{pH}$ independent drug release but also results in incomplete drug release. Thus, the formulation must be optimized for required release profile keeping the total amount of drug release in mind. 
Complexation of basic drugs with carbopol in basic media is not a universal phenomenon. Complexation depends on following factors [37]:

i. Shielding of basic groups in the drug molecule. If basic groups of the drug molecule are shielded by non-ionizing functional groups, then it will reduce the chances for complexation.

ii. pKa of the drug molecule. Weakly basic drugs undergo complexation to less extent compared to strongly basic drugs. For example, Verapamil Hydrochloride has pKa of 9.04 and Papaverine Hydrochloride has pKa of 6.8. In comparision, Verapamil Hydrochlorde undergoes complexation to high extent compared to Papaverine Hydrochloride.

iii. $\mathrm{pH}$ of the media and microenvironment must support the complexation. For effective complexation, the drug and carbopol must be completely ionized. Extent of ionization of drugs depends on the $\mathrm{pH}$ of the medium. Most of the basic drugs exist in completely ionized state in acidic $\mathrm{pH}$. However carbopol remains unionized in acidic $\mathrm{pH}$. Maximum swelling and ionization of carbopol resins is reported to occur in $\mathrm{pH} 5$ to 9 . Thus, for formation of drug-carbopol complex, the drug must be in completely ionized state in the $\mathrm{pH}$ values 5 to 9 . At these conditions, the swelling of carbopol resin is maximum thus exposing ionized carboxylic acid groups for complexation with drug molecules. For example, Propranolol hydrochloride is a basic drug with $\mathrm{pKa}$ of 9.5. At acidic $\mathrm{pH}$ proproanolol is completely ionized. At $\mathrm{pH} 4.5$ carbopol starts to ionize and complex with the ionized drug. At $\mathrm{pH} 7.5,97 \%$ of carbopol is ionized and $99 \%$ of propranolol is ionized. Thus, the Propranolol - Carbopol complex formation is high in intestinal $\mathrm{pH}$ rater than gastric $\mathrm{pH}$. 
iv. Orientation and spatial arrangement of carboxylic acid groups in hydrated polymer can influence the extent of complexation. For example, carbopol 934, 940 and 941 have similar carboxylic acid content. However amount of polymer required for the formation of saturated drug-polymer complex is significantly different. This implies orientation of carboxylic acid groups influence the extent of complexation. Orientation of carboxylic acid groups in hydrated polymer depends on the extent of cross linking. Carbopol grades with high degree of cross linking don't "open up" upon hydration as the cross links constrain the uncoiling of the polymer chains. Thus, higher the cross linking less the chances for complexation.

\subsubsection{Bioadhesion characteristics and drug release}

Carbopol is an excellent bioadhesive. Bioadhesive nature of carbopol helps to increase the bioavailability of the drugs [38]. When incorporated in tablets, carbopol increases the gastro intestinal transit time. Increase in gastric intestinal time improves drug absorption by keeping the tablet in regions of gastro intestinal tract where the drug absorption is high. Use of carbopol as bioadhesive to sustain the gastric transit time and improve the efficiency of controlled release systems is one of the extensively studied topics [38-71].

Y. Capan et al [72] showed that sustained release acetaminophen tablets containing carbopol 934P as release controlling polymer provided more uniform excretion rate than conventional controlled release tablets containing cellulose polymers as release controlling polymer. A similar enhancement in bioavailability was observed by K. Ciftci [73] with lithium carbonate, a sparingly soluble drug and using carbopol 934P as sustained release matrix. Hence, it appears that enhancement in bioavailability is not a drug specific phenomenon however more 
general and can be attributed to carbopol's ability to increase the gastro intestinal transit time. In a following paper, to illustrate the effect of tablet excipients on the improvement of bioavailability with carbopol tablets, K. Ciftci [73] et al compared formulations containing water insoluble fillers (Dibasic calcium phosphate, mirocystalline cellulose) and water soluble fillers (lactose and dextrates). Formulations containing water insoluble fillers showed poor bioavailability than conventional formulations containing cellulose polymers while formulations containing water soluble fillers showed similar bioavailability compared to conventional formulation. However, in formulations containing water soluble fillers, peak height of urinary excretion curve was low and the time required to reach peak height is longer indicating the fact that side effects of the drug may be low with carbopol formulations containing water soluble fillers. This finding implies that enhancement in performance of drug delivery system can be observed not only by increase in drug bioavailability with carbopol based polymeric system but also decrease in side effects of the drugs. These effects are related to other excipients in the formulation. Thus, in carbopol based tablet formulation the excipients must be selected not only to achieve an optimum drug release profile but also to maximize therapeutic potential or to minimize the side effects of the drug.

In vitro dissolution test can serve as an indicator for extent of urinary excretion of drug from carbopol based sustained release tablets. Perez Marcos [30] et al developed a correlation between in vitro dissolution and urinary excretion of atenolol tablets containing carbopol 934P as release controlling polymer.

Bioadhesive property of carbopol makes it suitable excipient in buccoadhesive tablet formulations [41, 42, 56, 57, 59, 60, 74-81], vaginal formulations [48, 49, 71, 82-84], ocular formulations $[64,66,85,86]$. In general, carbopol is blended with other controlled release 
polymer to optimize the drug release profiles. For, although carbopol is a good sustained release polymer, the gel strength of carbopol is too strong to release drugs with poor solubility. Therefore, combination of carbopol and other soluble linear cellulosic polymers are used in buccoahesive formulations.

\subsubsection{Release kinetics}

As discussed earlier, peppas model explain the release mechanism involved in the drug release. The release exponent " $n$ " in peppas equation, based on the geometry of the device, can be used to identify the release mechanism. Peppas equation can explain drug delivery systems with three different geometries i.e., slab, sphere and cylinder. Tablets prepared with flat faced punches can be considered as slabs. In systems with slab geometry, $\mathrm{n}=0.5$ indicates fickian diffusion, $0.5<\mathrm{n}<1$ indicates non-fickian (anomalous) transport, $\mathrm{n}=1$ indicates case II transport (zero order drug release/time independent), $\mathrm{n}>1$ indicates super case II transport. In non-fickian type diffusion, rapid release rate is observed in the first phase of the dissolution then the release rate tails of with time. Combination of diffusion and polymer swelling can cause this type of anomalous transport. The release exponent values may differ based on type of carbopol used in the formulation, $\mathrm{pH}$ of the dissolution media, drug solubility etc. This implies the release mechanism is function of multiple factors and drug specific. Generally drug release from carbopol follows non-fickian release kinetics. However, drug solubility, $\mathrm{pH}$ of the dissolution media, ionic characteristics of the drug and other excipients in the formulations can significantly influence the drug release kinetics of carbopol matrix [37, 70, 87-93].

For example, poorly soluble theophylline was formulated in sustained release tablet formulation using carbopol 934P, the release exponent of the formulation in SGF was 0.73 and 
in SIF it was 0.93. This implies, swelling was more predominant mechanism of drug release in SIF compared to SGF.

\subsubsection{Excipients in controlled release formulations}

Excipients used in the formulations containing carbopol as release controlling matrix can have significant impact on controlled release property of the matrix [23, 30, 94]. Carbopol is compatible with most of the commonly used excipients such as anhydrous lactose, hydrated lactose, dicalcium phosphate, talc, magnesium stearate and starch. Freely water soluble excipients such as sugars must be avoided. For, upon dissolution the osmotic pressure created by sugars can break the carbopol matrix. Lactose was a better excipient compared to microcrystalline cellulose and starch. Microcrystalline cellulose can absorb lot of water into the tablet effectively ruining carbopol's ability to retard the water uptake. Thus, formulations containing microcrystalline cellulose will require more carbopol compared to formulations containing dicalcium phosphate or lactose. This will increase overall cost of the formulation.

Y. Capan et al [95] compared the use of dibasic calcium phosphate, microcrystalline cellulose, lactose and dextrates as fillers in sustained release formulation of lithium carbonate. Tested for their controlled release properties, formulations showed no significant difference in their prolongation of drug release. However, a significant difference is observed between water soluble fillers and water insoluble fillers in the urinary excretion of the drug. Formulations containing water insoluble fillers (dicalcium phosphate, micro crystalline cellulose) showed $8 \%$ less in average total drug excretion compared to formulations containing water soluble fillers (lactose and dextrates). This implies that invitro differences in the release profile from carbopol matrix are not an indicator of the biological performance of the formulations. 
Dicalcium phosphate, water insoluble filler, can prolong the dissolution times of carbopol matrix compared to other water insoluble and soluble fillers. This is attributed to its aqueous solubility and inability to absorb water unlike micro crystalline cellulose. It noteworthy to mention that carbopol matrix doesn't dissolve or disintegrate upon ingestion. They "deaggregate" and become individual gel micro particles then eliminated from the body. Before being eliminated, the individual gel particles can control the drug release. Thus, unlike cellulose ethers, carbopol based system can control the drug release not only when it was in intact shape but also after deaggregation into gel particles.

Sustained release carbopol tablets containing starch as filler had poor sustained release properties. Starch is water swellable thus tablet swelling is extremely high resulting in tablet disintegration.

Carbopol can be processed with other excipients to prepare co-processed excipients [9698]. Such co-processed excipients were found to have superior bioadhesive and controlled release properties. In spite of research based evidence on advantages of preparing novel coprocessed excipient, no commercial product is available.

\subsubsection{Modifying release profiles in carbopol matrix}

Drug release rate from carbopol matrix can be modified by selection of appropriate polymer grade and co-excipient in the formulation. Appropriate carbopol grade must be selected based on the drug solubility and requirement on duration of drug release.

Slow drug release kinetics can be achieved with carbopol 971P or carbopol 934P. In general, increasing the polymer concentration reduces the drug release. The effect of carbopol concentration on the drug release depends on the filler used in the formulation. Non-swellable, 
insoluble filler like dicalcium phosphate can prolong the dissolution time. Non-swellable, water soluble filler like lactose can provide shorter dissolution time. Thus, fine tuning of dissolution rate can be achieved by selection of appropriate filler or by combining fillers in the formulation.

\subsubsection{Extrusion spheronization}

As described earlier, water is a good plasticizer for carbopol. Glass transition temperature of carbopol reduces below room temperature when carbopol comes in contact with water. This makes carbopol based formulations very water sensitive. In extrusion spheronization process, a wet mass is made using aqueous fluids (water or binder solution) and extruded then spheronized. In general extrusion spheronization formulations use 30 to $40 \%$ of water as wetting fluid for preparing wet mass. However, if the formulation contains carbopol, then use of water as wetting fluid is impossible. In such cases, salt solutions can be used as wetting fluids [99].. Salt solutions reduce tackiness of carbopol. Salt concentration in the solution must be optimized to minimize the tackiness. Calcium chloride salt solution is commonly used as wetting fluid for formulations containing carbopol. Calcium chloride concentration is salt of choice as formulations prepared using calcium chloride salt solution yield pellets with superior roundness and smoothness. Caclium chloride reduces the tackiness by complexing with ionized carboxylic acid groups. Calcium ions are bivalent cations and it can complex with two carboxylic acid anions. This complex nullifies the tackiness of carbopol.

Few studies report use of carbopol in melt extrusion formulations [46, 100]. However, in melt extrusion formulation carbopol is generally used with other excipients in small quantities to improve either bioadhesivness of the formulations or to improve the efficiency of the controlled 
release polymer. Carbopol has versatility to be processed in melt extrusion formulations. In future, novel carbopol based formulations can be developed using melt extrusion technique.

It is our objective to find a method that can improve the usage of carbopol in pharmaceutical industry. Once if such method is identified, it was also our interest to develop controlled release matrix formulations using carbopol as release controlling polymer. 


\section{Chapter 2: Evaluation of Granular Characteristics of Carbopol-Wax Blend}

\subsection{INTRODUCTION}

In situ hot melt granulation technology [101] is one of the quickest and simplest techniques available to prepare sustained release matrix granules. In this technique a waxy material is used as binder. It is uniformly blended with the drug, polymer and filler before granulation. During granulation, temperature of the granulator is raised above the melting point of the wax. Upon melting waxes form a viscous, sticky solution in the granulator to which the powder particles in the blend adhere and form agglomerate. Owing to the high shear mixing action in the granulator, the agglomerate is broken into smaller granules. Thus, the granule formation, breaking and size growth happen simultaneously as the waxy binder melts. Thus, this

process is difficult to model. However, there are many process and formulation variables that can influence the physical properties of granules obtained. The physical property of granules is influenced by particle size of the waxy binder, jacket temperature, mixing speed, binder concentration, granulator load etc.

Carbopol is cross linked poly acrylic acid, commercially available as many grades based on its degree of crosslinking. Although there are reports on use of Carbopol in preparation of hydrophilic matrix systems for oral controlled drug delivery, there are many process difficulties in handling this polymer in industrial setting. Carbopol is commercially available as fine powder with particles containing mean diameter of 0.2 microns. Owing to its fine particulate nature and static charge development it generally forms aggregates and mean diameter of these aggregates is 8 to 15 microns. Carbopol does not flow well because of its fine particulate nature, low density and static charge development. Carbopol is very sticky and it sticks with any kind of surface. 
Carbopol is also available in granular grades that have bigger mean particle diameter, faster flow. However, there are different problems with granular carbopol grades. They are: (i) Less efficency i.e., Efficiency of carbopol to control the drug release is higher when it is used as fine particles as fine particles can form more intact gel structure. (ii) Use of granular grade does not solve stickiness problem.

Hot melt granulation of carbopol with waxes can improve its flow characteristics and reduce the stickiness of carbopol. Since hot melt granulation is an in situ granulation technique, the powder grade of carbpol can be used thus maximum efficiency of the polymer to control the drug release can be attained. In this section, the development of hot melt granulation technique for preparing carbopol granules with Gelucire 50/13 is presented. Gelucire 50/13 is blend of poly ethylene glycol esters of long chain fatty acids and available as waxy solid. Based on the literature on in situ hot melt granulation, it was hypothesized that process conditions significantly influence the physical properties of granules. It was our interest to evaluate the effect of jacket temperature, mixing speed and massing time on the physical characteristics of the granules. This will aid in the development of robust granulation technique for preparing carbopol granules using a waxy binder.

\subsection{MATERIALS AND METHODS}

\subsubsection{Materials}

Our primary materials in this study were obtained from commercial sources. They were Carbopol 971P (Noveon, Cleveland, OH), Gelucire 50/13 (Gattefosse, Paramus, NJ). 


\subsubsection{Methods}

\subsubsection{Preparation of carbopol-wax blend}

Carbopol-wax granules were prepared using in situ hot melt granulation. Sixteen batches were prepared using different process conditions. Process conditions for preparing the batches are given in Table 2.1. Batch size for all batches was 300 gms. Pre-weighed quantities of carbopol 971P and the waxy binder were added to the Robot Coupe ${ }^{\circledR}$ (Jackson, MS) high shear granulator. Granulator blade was operated in forward mode for mixing the contents prior to granulation. Mixing was done at 1500 rpm blade speed for 2 minutes. Following mixing, a circulating water bath was attached to granulator. Temperature of water bath was set according to the conditions required for each batch. Temperature of water jacket of the granulator was maintained constant throughout granulation as per conditions required for each batch.

Temperature of water bath was monitored using an external digital thermometer by placing the temperature probe of the thermometer in the sleeve in the jacket. Granulation speed and time were also set according to the conditions required for each batch. Granulation was done for specified time as per specifications for each batch. After granulation, the granules were allowed to cool to room temperature by spreading them on metal trays.

\subsubsection{Bulk density evaluation}

Bulk density of the granules was evaluated using a $50 \mathrm{ml}$ measuring cylinder and a balance. Initially the weight of the measuring cylinder was tarred. Then, the granules were poured into the measuring cylinder till the fill volume was $40 \mathrm{ml}$ using a funnel. Then the weight of the measuring cylinder with granules was taken. This gives the weight of the granules. 
Table 2.1. Process conditions used for preparing carbopol-wax blends

\begin{tabular}{cccc}
\hline Batch code & $\begin{array}{c}\text { Granulation } \\
\text { temperature }\left({ }^{\circ} \mathrm{C}\right)\end{array}$ & $\begin{array}{c}\text { Mixing speed } \\
(\mathrm{RPM})\end{array}$ & Mixing time (Min) \\
\hline B - & 60 & 1000 & 1.5 \\
B - 2 & 60 & 1000 & 2 \\
B - 3 & 60 & 2000 & 1.5 \\
B - 4 & 60 & 2000 & 2 \\
B - 5 & 65 & 1000 & 1.5 \\
B - 6 & 65 & 1000 & 2 \\
B - 7 & 65 & 2000 & 1.5 \\
B - 8 & 65 & 2000 & 2 \\
B - 9 & 70 & 1000 & 1.5 \\
B - 10 & 70 & 1000 & 2 \\
B - 11 & 70 & 2000 & 1.5 \\
B - 12 & 70 & 2000 & 2 \\
B - 13 & 75 & 1000 & 1.5 \\
B - 14 & 75 & 1000 & 2 \\
B - 15 & 75 & 2000 & 1.5 \\
B - 16 & 75 & 2000 & 2 \\
\hline
\end{tabular}


Bulk density of the granules was calculated using Eq. 2.1 and the bulk density of the formulations is presented in Table 2.2.

Bulk density $=$ Weight of granules $/$ Volume of granules

\subsubsection{Granule size evaluation}

The particle size and size distribution of each material in the formulation were determined by the Gilsonic Autosiever (Gilson Company, Inc., Worthington, OH). A series of sieves with varying mesh size number ranging from 20 to 200 were used in this study. Prior to the experiment, each sieve was weighed and the initial weight was recorded. Approximately ten grams of sample were then placed on the top sieve (20 mesh), which was closed by rubber gasket and spacer. Stack of sieves was then placed onto the shaker and tightened. The stack was mechanically shaken for a total time of 7 minutes. This total time was divided into 3 parts, time A, B and C. Time A ( 60 seconds) was the time required to increase the speed from zero speed to the predetermined shaking speed, whereas time B, which was set for 5 minutes, was the time maintained at the maximum amplitude. Time $\mathrm{C}$ (60 seconds) was the time required for the decrease of speed from the maximum amplitude to zero speed.

Once the cycle is completed, the lock was loosened and the stack of sieves was removed from the shaker. The final weight of each sieve and the retained sample on each sieve and the sieve was weighed again together and the weight was recorded and the percentage of material retained on each sieve was calculated. Sieve shaker used in this study uses both ultra sonic vibrations and mechanical agitation to segregate granules according to their size. Use of ultra sonic sound for granule size separation minimizes destruction of granules during the test. 
Table 2.2. Physical characteristics of carbopol-wax blends

\begin{tabular}{|c|c|c|c|c|c|}
\hline \multirow{2}{*}{$\begin{array}{l}\text { Batch } \\
\text { code }\end{array}$} & \multirow{2}{*}{$\begin{array}{c}\text { Bulk } \\
\text { density* } \\
\text { (gms/ml) } \\
\end{array}$} & \multicolumn{3}{|c|}{ Granule size distribution $(\%)^{*}$} & \multirow{2}{*}{$\begin{array}{l}\text { Dynamic } \\
\text { flow rate* } \\
(\mathrm{gms} / \mathrm{sec})\end{array}$} \\
\hline & & Coarse & Medium & Fine & \\
\hline $\mathrm{B}-1$ & $0.276 \pm 0.02$ & $4.34 \pm 2.11$ & $19.75 \pm 1.79$ & $75.91 \pm 0.28$ & $8.14 \pm 0.51$ \\
\hline $\mathrm{B}-2$ & $0.376 \pm 0.05$ & $8.81 \pm 1.23$ & $28.50 \pm 0.91$ & $62.69 \pm 2.39$ & $13.60 \pm 0.41$ \\
\hline $\mathrm{B}-3$ & $0.315 \pm 0.02$ & $6.97 \pm 1.50$ & $18.72 \pm 1.55$ & $74.32 \pm 1.56$ & $16.15 \pm 0.59$ \\
\hline$B-4$ & $0.326 \pm 0.05$ & $26.68 \pm 0.82$ & $44.42 \pm 0.59$ & $28.89 \pm 1.13$ & $13.26 \pm 0.98$ \\
\hline$B-5$ & $0.313 \pm 0.03$ & $2.95 \pm 0.35$ & $18.14 \pm 1.25$ & $78.91 \pm 0.53$ & $16.62 \pm 0.52$ \\
\hline$B-6$ & $0.414 \pm 0.03$ & $13.06 \pm 2.61$ & $32.13 \pm 2.85$ & $54.82 \pm 2.75$ & $19.64 \pm 0.59$ \\
\hline $\mathrm{B}-7$ & $0.380 \pm 0.02$ & $6.37 \pm 2.09$ & $22.61 \pm 1.82$ & $71.03 \pm 0.40$ & $20.35 \pm 1.49$ \\
\hline $\mathrm{B}-8$ & $0.374 \pm 0.04$ & $54.59 \pm 2.96$ & $37.19 \pm 2.09$ & $8.23 \pm 2.77$ & $12.43 \pm 0.59$ \\
\hline $\mathrm{B}-9$ & $0.442 \pm 0.04$ & $11.24 \pm 0.14$ & $35.15 \pm 0.51$ & $53.61 \pm 1.60$ & $14.90 \pm 0.94$ \\
\hline$B-10$ & $0.429 \pm 0.02$ & $46.61 \pm 1.33$ & $44.02 \pm 1.10$ & $9.37 \pm 2.71$ & $13.42 \pm 0.67$ \\
\hline$B-11$ & $0.394 \pm 0.06$ & $32.99 \pm 1.68$ & $45.43 \pm 1.22$ & $21.58 \pm 2.84$ & $14.11 \pm 0.47$ \\
\hline$B-13$ & $0.511 \pm 0.05$ & $15.16 \pm 0.23$ & $36.14 \pm 0.10$ & $48.70 \pm 2.68$ & $23.40 \pm 1.17$ \\
\hline$B-14$ & $0.429 \pm 0.09$ & $61.39 \pm 1.83$ & $33.58 \pm 0.49$ & $5.03 \pm 2.59$ & $13.29 \pm 0.48$ \\
\hline
\end{tabular}

*indicates values presented in the table are average of three measurements. Average values are presented with standard deviation of three measurements. 
Based on the retaining of the granules, they were divided into three categories; (i) Coarse granules (percent weight of granules retained on no. 30 sieve), (ii) Medium size granules (percent weight of granules that pass through no. 30 sieve and retained on no. 60 sieve, (iii) Fine granules (percent weight of granules that pass through no. 60 sieve). Percent weight of coarse, medium size and fine granules is presented in Table 2.2.

\subsubsection{Dynamic flow rate evaluation}

Dynamic flow of the material was determined using a Hanson Flowdex (Hanson Research Corporation, Chatsworth, CA). The device consists of a metal cylinder attached to a metal plate with an orifice at the bottom. This orifice was closed or opened by the movable stage attached to the bottom of the plate. Material was filled into the cylinder with the orifice closed. Approximately three fourth of cylinder was filled with the material. Once filled, the movable stage blocking the orifice was removed from the bottom of the plate and the material was allowed to flow through the orifice onto a balance, which was connected to a computer. The dynamic weight change of material that flows through the orifice and time in millisecond were recorded electronically using Software WinWedge ${ }^{\circledR}$ (TAL Technologies, Inc., Philadelphia, PA). The data obtained was plotted as the weight of the material versus time. The flow rate was then determined as the slope of the linear regression of the plot. Dynamic flow rate of all formulations is presented in Table 2.2. 


\subsection{RESULTS AND DISCUSSION}

\subsubsection{Study design}

Objective of the study was to evaluate the effect of mixing speed and massing time of granulation at different jacket temperatures. Asymmetrical factorial design $\left(4^{1} \mathrm{X} 2^{2}\right)$ with four different jacket temperatures and two levels (low, high) of mixing speed and massing time was used in this study.

\subsubsection{Bulk density}

Effect of mixing speed and massing time on bulk density of the granules at different jacket temperature is depicted in Figure 2.1. At $60^{\circ} \mathrm{C}$ jacket temperature and $1000 \mathrm{rpm}$ mixing speed, increasing the massing time to high level increased the bulk density of the granules by $36.14 \%$. However, at $60^{\circ} \mathrm{C}$ jacket temperature and $1000 \mathrm{rpm}$ mixing speed, increasing the massing time to high level increased the bulk density by $3.42 \%$ and this increase is statistically insignificant $(\mathrm{P}>0.05)$. This implies that there is a limit of densification achieved at higher speeds and this limit is reached at low massing time. This could be attributed to the high shear force created at high mixing speed. At higher shear force, the size growth is faster compared to lower speeds. However, the granule size increases with no significant increase in density of the granules. This could be due to the formation of rubbery granules at higher speed. At $65^{\circ} \mathrm{C}$ jacket temperature and $1000 \mathrm{rpm}$ mixing speed, increasing the massing time to high level increased the bulk density of the granules by $32.48 \%$. This is less compared to the change in bulk density observed at $60^{\circ} \mathrm{C}$ in same mixing conditions. At $65^{\circ} \mathrm{C}$ jacket temperature and $2000 \mathrm{rpm}$ mixing speed, increasing the massing time decreased the bulk density by $1.45 \%$. 


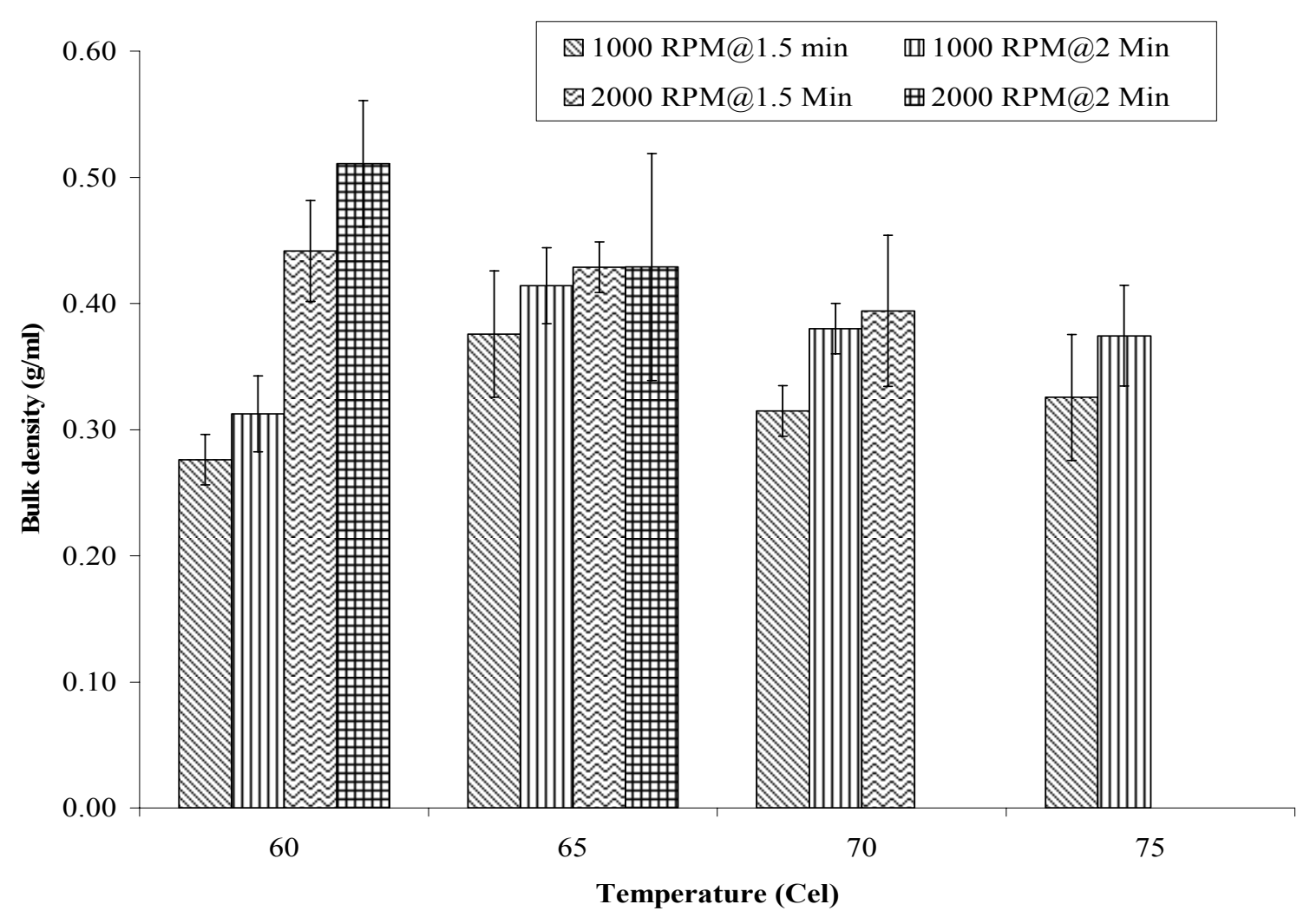

Figure 2.1. Effect of process conditions on bulk densities of formulations $\uparrow$

$\uparrow$ Each bar represents average of three measurements. Standard deviation of three measurements is presented as error bars. 
Although this change is statistically insignificant $(\mathrm{P}>0.05)$, it implies formation of rubbery granules at higher speed. Results indicate formation of rubbery is dependent on the mixing speed and temperature. This is further supported by granules prepared at $70^{\circ} \mathrm{C}$ and $75^{\circ} \mathrm{C}$. At $70^{\circ} \mathrm{C}$ jacket temperature and $1000 \mathrm{rpm}$ mixing speed, increase in bulk density was $20.69 \%$ (lower compared to similar conditions at $60^{\circ} \mathrm{C}$ and $65^{\circ} \mathrm{C}$ ). At $75^{\circ} \mathrm{C}$ jacket temperature and 1000 rpm mixing speed, increase in bulk density was $15.01 \%$. This shows that formation of rubbery granules is dependent on the jacket temperature.

At $70^{\circ} \mathrm{C}$ jacket temperature and $2000 \mathrm{rpm}$ mixing speed, increasing the granulation time resulted in formation of rubbery mass. And, at $75^{\circ} \mathrm{C}$ jacket temperature and $2000 \mathrm{rpm}$ mixing speed, rubbery mass was formed at low and high massing times. This clearly shows formation of rubbery granules/mass is dependent on jacket temperature and mixing speed. Based on jacket temperature and mixing speed settings, the massing time must be optimized to get granules of desired characteristics. For example, at high jacket temperature and high mixing speed, the massing time must be low to obtain discrete, free flowing granules.

\subsubsection{Granule size distribution}

Granule size distribution was non-normally distributed in most of the batches. Thus, geometric mean diameter could not be used for identifying the effect of process conditions on granule size growth. Since the size distribution was analyzed using sieves, three sieve cuts have been defined: coarse, moderate and fine. Granules retained on no. 30 sieve were considered coarse, granules that pass no. 30 sieve and retain on no. 60 sieve were considered acceptable and granules that pass through no. 60 sieve were considered fine. Granule size growth in insitu hot melt granulation is a complex process. It depends on particle size and size distribution of the 
components of the blend and melting point of the wax, interaction between wax and other excipients etc.

\subsubsection{Coarse granules}

Effect of temperature and process conditions on the amount of coarse granules is depicted in Figure 2.2. At $60^{\circ} \mathrm{C}$, when blade speed was $1000 \mathrm{rpm}$ and the granulation time was increased from low to high, there was $102.98 \%$ increase in the amount of coarse granules. However, at $60^{\circ} \mathrm{C}$, when the blade speed was $2000 \mathrm{rpm}$ and the granulation time was increased from low to high, there was $282.90 \%$ increase in the amount of coarse granules. This indicates that the size growth of the granules at a given jacket temperature is dependent on both mixing speed and massing time. The rate of size growth is faster at higher mixing speeds.

A similar trend was observed at $65^{\circ} \mathrm{C}$ jacket temperature, however, the magnitude of the size growth was higher than one observed at $60^{\circ} \mathrm{C}$. At $60^{\circ} \mathrm{C}$ jacket temperature and $1000 \mathrm{rpm}$ blade speed, when the granulation time was increased from low to high, there was $342.69 \%$ increase in the amount of coarse granules. At $65^{\circ} \mathrm{C}$ jacket temperature and $1000 \mathrm{rpm}$ blade speed, when granulation time was increased from low to high there was $757.30 \%$ increase in the amount of coarse granules. This indicates that the extent of coarse granules formed is dependent on mixing speed, massing time and jacket temperature. There is a significant interaction exist among these variables. Effect of mixing speed and mixing time on the extent of coarse granules formed will be higher at high jacket temperature. This can be attributed to the faster melting of wax at higher temperature.

Similar trend was observed with $70^{\circ} \mathrm{C}$ and $75^{\circ} \mathrm{C}$ jacket temperatures with few exceptions. At $70^{\circ} \mathrm{C}$, when mixing speed was $2000 \mathrm{rpm}$, increase in massing time turned the blend rubbery. 


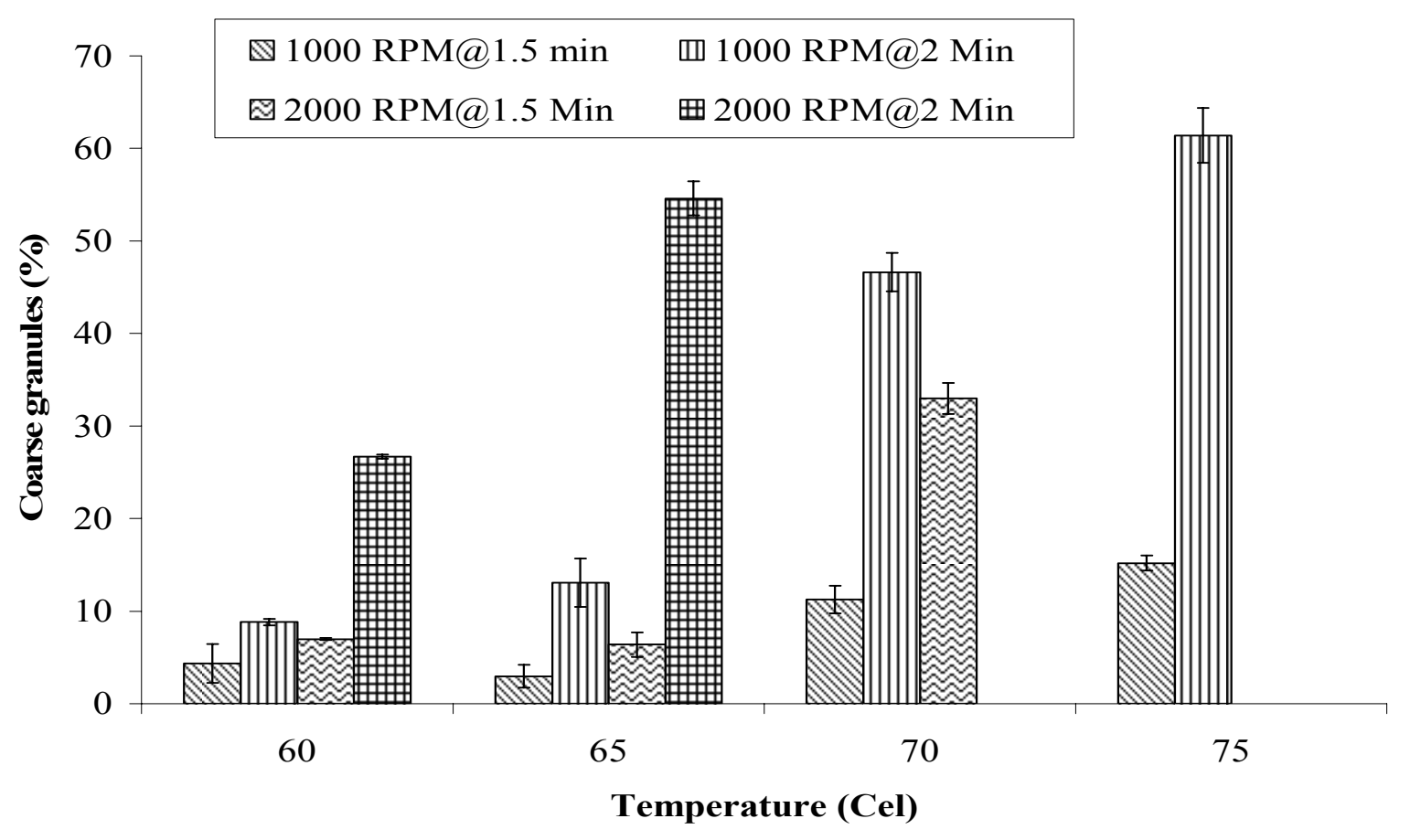

Figure 2.2. Effect of process conditions on the amount of coarse granules $\uparrow$

$\dagger$ Each bar represents average of three measurements. Standard deviation of three measurements is presented as error bars. 
This also implies that there is an interaction between Gelucire and carbopol 971P and this interaction results in formation of rubbery product. Formation of rubbery product in granulation is hastened by jacket temperature and mixing speed.

\subsubsection{Medium size granules}

Effect of temperature and process conditions on the amount of medium size granules is depicted in Figure 2.3. At $60^{\circ} \mathrm{C}$ jacket temperature and $1000 \mathrm{rpm}$ mixing speed, increasing the massing time from low to high increased the amount of acceptable granules by $44.30 \%$. However, at $60^{\circ} \mathrm{C}$ jacket temperature and $1000 \mathrm{rpm}$ mixing speed, increasing the massing time from low to high increased the amount of acceptable granules by $137.35 \%$. This indicates that formation of acceptable size granules depends on both processing speed and time. High mixing speed and longer mixing time at low jacket temperature yield more amounts of medium size granules.

At $65^{\circ} \mathrm{C}$ jacket temperature and $1000 \mathrm{rpm}$ mixing speed, increasing the massing time from low to high increased the amount of medium size granules by $77.08 \%$. This is higher compared to similar processing conditions at $60^{\circ} \mathrm{C}$. This implies at $1000 \mathrm{rpm}$, increasing jacket temperature and mixing time will yield higher amount of medium size granules. However, the trend was different at $2000 \mathrm{rpm}$. At $2000 \mathrm{rpm}$, increasing massing time increased the amount of medium size granules in the blend by $64.51 \%$. This is significantly lower compared to similar conditions at $60^{\circ} \mathrm{C}$ where $137.35 \%$ increase was observed. At $70^{\circ} \mathrm{C}$ and $1000 \mathrm{rpm}$ mixing speed, amount of medium size granules increased by $25.23 \%$. However, at $75^{\circ} \mathrm{C}$ and $1000 \mathrm{rpm}$ processing speed, amount of acceptable granules decreased by $7.08 \%$. 


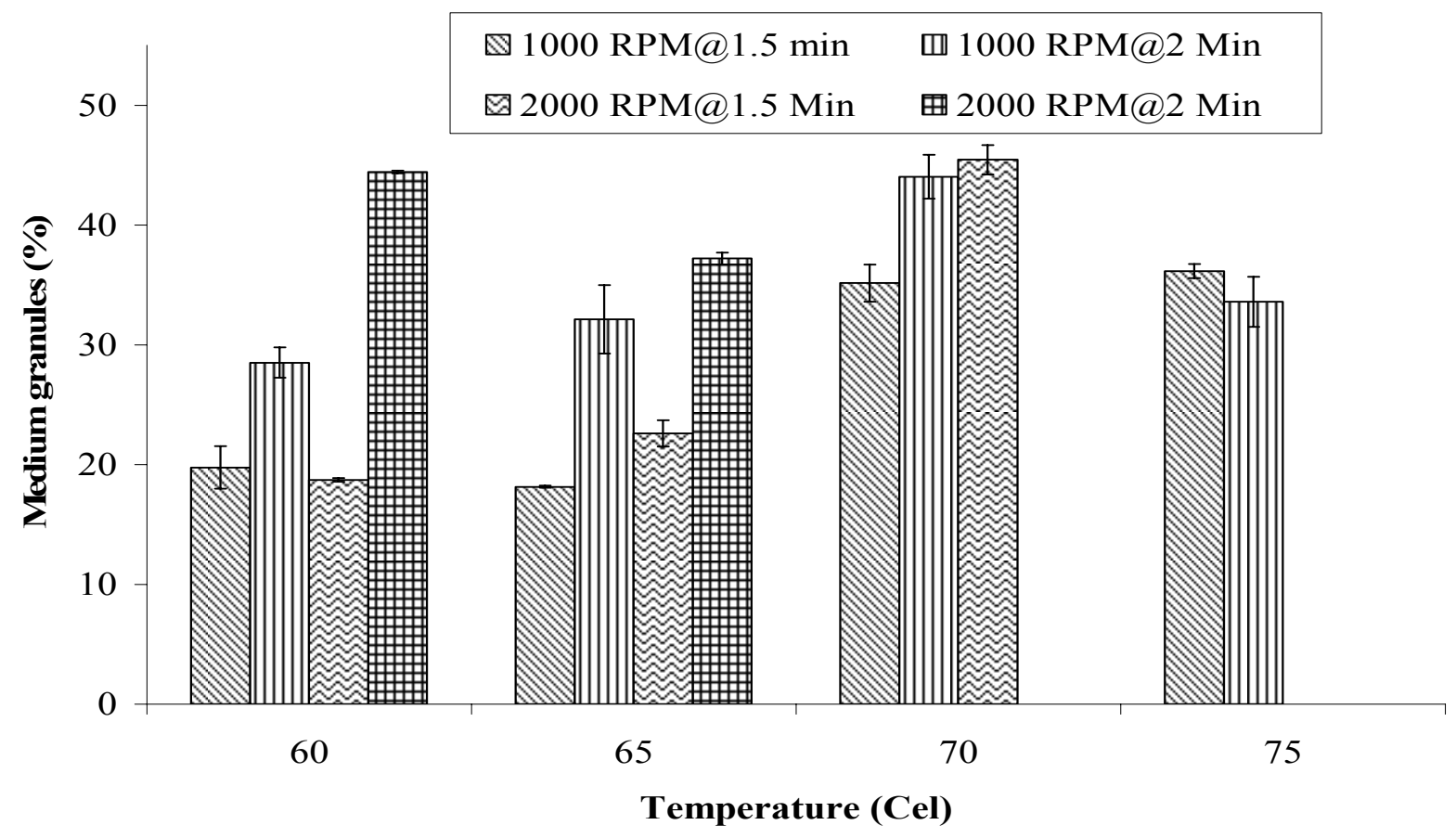

Figure 2.3. Effect of process conditions on the amount of medium size granules $\dagger$

$\dagger$ Each bar represents average of three measurements. Standard deviation of three measurements is presented as error bars. 
This implies: (i) size growth does not occur uniformly at all jacket temperatures, (ii) mixing speed and massing time have significant interaction with jacket temperature in determining the formation of amount of medium size granules.

\subsubsection{Fine granules}

Amount of fine granules is associated with the amount of coarse and acceptable granules formed and the amount of size reduction during the granulation. For, during in situ hot melt granulation, size growth and reduction take place simultaneously. Based on the processing conditions extent of size growth and reduction will differ.

Effect of temperature and process conditions on the amount of fine granules is depicted in Figure 2.4. At $60^{\circ} \mathrm{C}$ jacket temperature and $1000 \mathrm{rpm}$ mixing speed, increasing the massing time to high level decreased the amount of fine granules by $17.41 \%$. However, at $60^{\circ} \mathrm{C}$ jacket temperature and implies that effect of jacket temperature on amount of fine granules is augmented by mixing speed. Aforementioned interpretations are supported by amount of fine granules formed at $70 \mathrm{oC}$ and $75^{\circ} \mathrm{C}$ at $1000 \mathrm{rpm}$ mixing speed when mixing time was increased. At $1000 \mathrm{rpm}$, when mixing time was increased, while amount of fine granules decreased by $82.52 \%$ at $70^{\circ} \mathrm{C}$ jacket temperature, amount of fine granules decreased by $89.67 \%$ at $75^{\circ} \mathrm{C}$.

Summarily, (i) amount of coarse, acceptable, fine granules formed is dependent on the jacket temperature, (ii) significant interaction exist between jacket temperature, mixing speed and mixing time. Generally, size growth is faster at higher temperatures and higher mixing speeds. And, size reduction was not observed at higher mixing speeds, (iii) at $70^{\circ} \mathrm{C}$ and $75^{\circ} \mathrm{C}$ jacket temperatures, the granulation process becomes sensitive to mixing speed and time. Increasing mixing time at $70^{\circ} \mathrm{C}$ and increasing mixing speed at $75^{\circ} \mathrm{C}$ will form rubbery mass. 


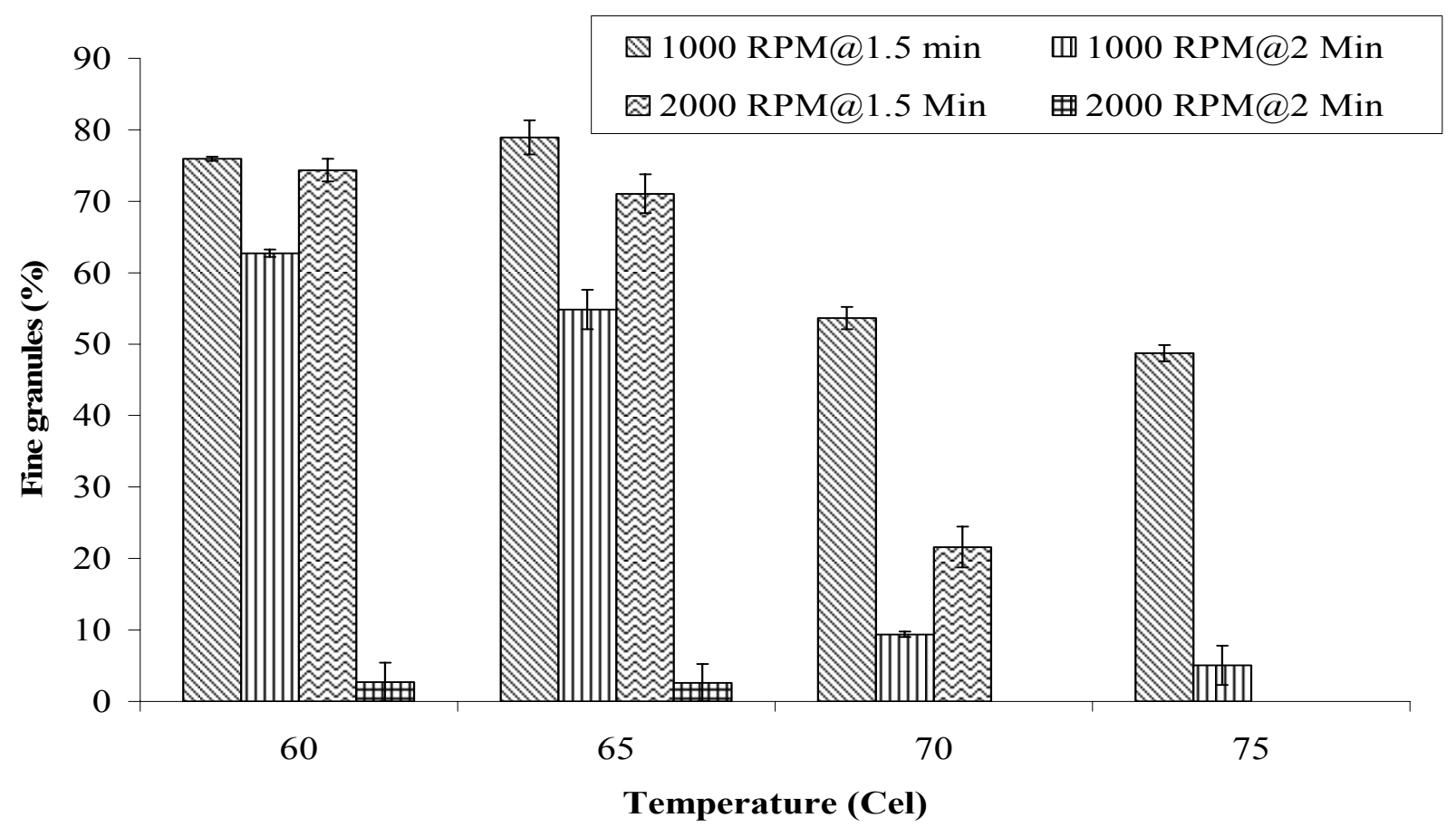

Figure 2.4. Effect of process conditions on the amount of fine granules $\uparrow$

$\dagger$ Each bar represents average of three measurements. Standard deviation of three measurements is presented as error bars. 


\subsubsection{Flow rate}

Flow rate of granules depends on granule size, size distribution and density of the granules. Effect of process conditions on the flow rate of granules is depicted in Figure 2.5. At $60^{\circ} \mathrm{C}$ jacket temperature and $1000 \mathrm{rpm}$ mixing speed, increase in massing time to high level increased the flow rate by $67.25 \%$. However, at $60^{\circ} \mathrm{C}$ jacket temperature and $2000 \mathrm{rpm}$ mixing speed, increase in massing time to high level decreased the flow rate by $18.06 \%$. This could be attributed to the density of the granules. Since rubbery and low density granules are formed faster at higher speed, flow rate and granular density could be related. A similar trend was observed at $65^{\circ} \mathrm{C}$ jacket temperature. At $65^{\circ} \mathrm{C}$ jacket temperature and $1000 \mathrm{rpm}$ mixing speed, increase in massing time to high level increased the flow rate by $18.12 \%$, which is less compared to the increase observed in same conditions at $60^{\circ} \mathrm{C}$. At $65^{\circ} \mathrm{C}$ jacket temperature and $2000 \mathrm{rpm}$ mixing speed, the flow rate decreased by $38.85 \%$. Batch prepared at $75^{\circ} \mathrm{C}$ jacket temperature with low mixing speed and low massing time had maximum flow rate $(23.38 \pm 1.16 \mathrm{gms} / \mathrm{sec})$. This formulation had maximum density among all formulations $(0.51 \pm 0.02)$. This implies that flow rate and density are related and formation of low density, rubbery granules will result in reduction in flow rate of granules.

\subsection{CONCLUSIONS}

Free flowing carbopol granules can be prepared using hot melt granulation process. Gelucire 50/13 can be used as binder for granulating carbopol in hot melt granulation process. Physical properties of carbopol - wax granules were dependent on the process conditions used to prepare them in high shear granulator. Effect of mixing speed and mixing time on the physical properties of granules was dependent on the granulation temperature. Increase in granulation 


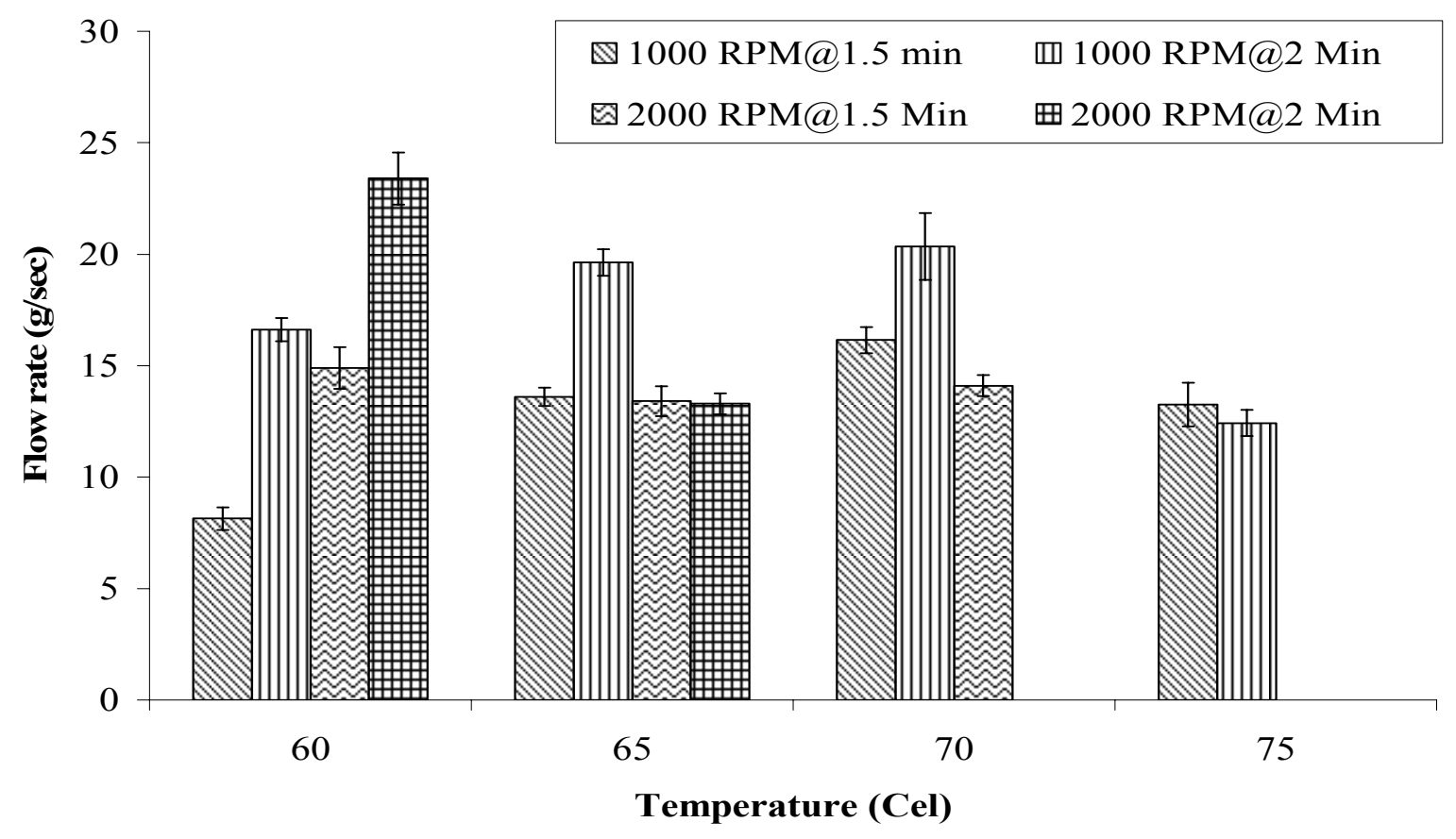

Figure 2.5. Effect of process conditions on the dynamic flow rate of the granules $\uparrow$

$\dagger$ Each bar represents average of three measurements. Standard deviation of three measurements is presented as error bars. 
temperature turns the granulation process sensitive to mixing speed and mixing time. Free flowing, discrete carbopol-wax granules can be prepared in various process conditions. 


\section{Chapter 3: Formulation Development of Propranolol Hydrochloride}

\subsection{INTRODUCTION}

Propranolol hydrochloride is a $\beta$-adrenergic blocking agent, i.e., a competitive inhibitor of the effects of catecholamines at $\beta$-adrenergic receptor sites. It is widely used in therapeutics for its antihypertensive, antiangorous and antiarrhythmic properties. Furthermore, it has a short elimination half-life of $3 \mathrm{~h}$, which makes it a suitable candidate to be delivered at a controlled rate.

Objective of this work is to develop sustained release matrix tablets of propranolol hydrochloride using carbopol - wax blends and to optimize the invitro drug release profiles according to USP criteria. The matrix tablets contained five components. They are

i. Therapeutic agent: propranolol hydrochloride.

ii. Release controlling polymer: carbopol 971P or carbopol 974P.

iii. Binder: glyceryl mono stearate or gelucire 50/13.

iv. Filler: dicalcium phosphate or lactose monohydrate.

v. pH modifiers: sodium carbonate and glycine.

Rationale for selection of aforementioned matrix component is given below.

\subsection{SELECTION OF MATRIX COMPONENTS}

\subsubsection{Selection of release controlling polymer}

Carbopols have been reported in the literature for their potential use in oral controlled release delivery systems. This is because of carbopol's ability to swell and form viscous gel in 
aqueous environment. Carbopols can absorb lot of water and form gels and these gels are called hydrogels. When formulated with drugs, the carbopol hydrogels can entrap the drug molecules and control their release from the delivery system. Carbopols are available in various grades suitable for pharmaceutical use. Carbopol 971P and Carbopol 974P are the most commonly used grades used in controlled release oral formulations. Carbopol 971P is less cross linked than carbopol 974P. The release retardant property of carbopol is directly related to its concentration in the formulation. In general, drug release rate is inversely proportional to the carbopol concentration. It is reported that carbopol 971P is more effective than carbopol 974P in oral controlled release formulations. This is attributed to free uncoiling of polymer chains of carbopol 971P and effective entrapment of drug molecules compared to carbopol 974P.

Carbopol is an anionic polymer. Sixty two percent of the polymer is made up of carboxyl groups. Hence, it has high potential for interaction with basic drugs. This ionic interaction may boost carbopol's controlled release potential i.e., may be effective in low concentrations compared to cellulose based polymers that have no interaction with basic drugs.

In spite of its efficiency, carbopols are not commonly used in oral controlled delivery systems. As a result, complete understanding of mechanism of drug release with respect to dynamic swelling has not been developed. Hence, carbopol has been selected for this study. The objective of the study is to develop an USP (United States Pharmacopoeia) compliant formulation of propranolol hydrochloride and to optimize the drug release profiles based on the mechanism of drug release.

A literature survey showed no supporting literature for formulation development of propranolol hydrochloride controlled release tablets using carbopol - wax blends. Carbopol is 
one of the most under utilized polymers in the pharmaceutical industry because of following reasons.

i. Carbopol is commercially available as fluffy powder with average particle diameter of 0.2 microns. Owing to its fine particulate nature, carbopols aggregate forming loose aggregates with average diameter of 8 to 30 microns. These loose aggregates have poor flow characteristics because of their low bulk density.

ii. Carbopol is a sticky polymer. It is an excellent bioadhesive and it sticks to any kind of surface. This stickiness creates process difficulty.

iii. Carbopol turns tacky in presence of water. Water is a good plasticizer for carbopol. When comes in contact with water, carbopol's glass transition temperature lowers below room temperature. Therefore, most commonly used wet granulation process can not be used with formulations containing carbopol.

Essentially, flow characteristics of carbopol must be improved and stickiness of the material must be reduced. Manufacturers of carbpol make granular grade of the material using roller compaction process. This granular grade has better flowability compared to powder grade. However, if granular grade of the carbopol is used in the formulation, then the efficiency of the polymer will be low. That is, more amount of polymer will be required to achieve controlled release properties similar to that of powder grade. Carbopol has unique gelling properties. Each carbopol particle has potential to form microgel and entrap the drug molecules. In granular grade, carbopol particles are compacted reducing individual polymer particle's ability to entrap the drug molecule. Thus, more polymer is needed to compensate this loss of carbopol's ability to control the drug release as polymer microgel. Hot melt granulation technique can solve this problem. Hot melt granulation is a granulation technique where no water is used for preparing 
granules so polymer sticking is not a problem. Prior to granulation, intimate mixing of carbopol and the drug is achieved in high shear granulator. This blending provides proximity of carbopol particles to the drug particles. Therefore, less carbopol concentration is sufficient to control the drug release compared to using carbopol granules.

\subsubsection{Selection of therapeutic agent}

Propranolol hydrochloride was selected as model drug for this study for following reasons.

i. Propranolol Hydrochloride has a high aqueous solubility. As a result, it is difficult to control its release from matrix tablets. However, if a matrix system is developed and the properties of system are understood, then it is comparatively easy to develop controlled drug delivery system for drugs with low solubility.

ii. Propranolol Hydrochlorde is categorized as Class I drug in BCS classification. Class I drugs have high solubility and high permeability. It has uniform absorption throughout the gastro intestinal tract. These properties make propranolol hydrochloride as a suitable candidate for oral controlled release system.

iii. Propranolol Hydrochloride is a basic drug $(\mathrm{pKa}=9.5)$ and carbopol is an acidic polymer. At intestinal $\mathrm{pH}, 7.2,99 \%$ of the drug exists in ionized form. At intestinal $\mathrm{pH}$, carboxylic groups of carbopol are ionized and possess negative charges. Hence, there is potential interaction between positively charged amino groups of propranolol and negatively charged carboxylic acid groups. This ionic interaction could retard the drug release. Thus it is of interest to develop a delivery system which can release the drug completely, complying with USP standards. 


\subsubsection{Selection of pharmaceutical excipients}

\subsubsection{Selection of binder}

In this study, besides developing a controlled drug delivery system for propranolol hydrochloride, the effect of two binders namely Glyeryl Mono Stearate and Gelucire 50/13 on the granular properties is investigated.

Binder for the formulation development was selected based on favorable granulation characteristics. The evaluation criteria for granules are predefined and evaluated for formulations containing carbopol $(10 \%)$ with binder $(10 \%)$ and filler $(80 \%)$. These formulations contained no drug as the interest was to develop general conclusions from the study rather than developing drug specific conclusions. The criteria for evaluation are

i. Dynamic flow rate of the formulation must be more than $25 \mathrm{gms} / \mathrm{second}$.

ii. Hardness of the tablets prepared from formulations using $1 / 4$ " punches must be at least $4 \mathrm{kps}$ at low $(0.5 \mathrm{mT})$ and high $(1 \mathrm{mT})$ compression forces.

Two binders evaluated for their potential use in the formulation are Glyceryl Mono Stearate and Gelucire 50/13. Glyceryl Mono Stearate is a hydrophobic binder. Melting point of Glyceryl Mono Stearate is 56 to $58^{\circ} \mathrm{C}$. It is available as flakes or free flowing powder. For the entire study, flakes were used. HLB value of Glyceryl Mono Stearate is 1.4 indicating hydrophobicity of the material. Glyeryl Mono Stearate was selected because of its hydrophobicity and its reported use in literature for preparing controlled release oral formulations. Being hydrophobic, Glyceryl mono stearate can retard the water influx into the delivery system. In addition, if used as binder in hot melt granulation, Glyceryl Mono Stearate can embed the drug particles and form a dispersion of drug particles in hydrophobic waxy matrix. This will hamper the availability of 
drug particles to dissolution media and indirectly help carbopol's ability to control the drug release.

Another waxy binder evaluated in the study was Gelucire 50/13. Gelucire is blend of Glyceryl esters of fatty acids of poly ethylene glycol. Gelucires are commercially available with two number suffix, for example 50/13. The first number 50 denotes the melting point of the wax and the second number 13 denotes the HLB value of the wax. Gelucires are available in various melting points and HLB values. Gelucires with lower HLB values are hydrophobic and are recommended for controlled release applications. However, despite of the objective to develop controlled release product, Gelucire 50/13, a hydrophilic wax, is used. Gelucire 50/13 is selected to represent hydrophilic waxes as Glyceryl Mono Stearate represents hydrophobic waxes.

\subsubsection{Selection of fillers}

Two fillers are evaluated for their suitability to be used in the formulation. They are dicalcium Phosphate and Lactose monohydrate. Dicalcium phosphate is water insoluble filler and has no water swellability. Lactose monohydrate is water soluble filler.

It is reported in literature that use of dicalcium phosphate boosts controlled release properties of drug delivery system. Being insoluble, dicalcium phosphate resists the water influx into the delivery system. Controlling water influx is the first step in developing an efficient drug delivery system. Efficient drug delivery system must not only control the water influx but also drug efflux. In addition, dicalcium phosphate has excellent flow and compression characteristics and low cost. Dicalcium phosphate is selected to represent a category of water insoluble, nonswellable fillers. 
Lactose monohydrate represents water soluble, non-swellable filler. Lactose is a natural disaccharide and prepared from milk. It is one of the most commonly used excipients in sustained and immediate release formulation. It is water soluble. It has solubility of $1 \mathrm{~g}$ in $4.63 \mathrm{ml}$ of water at room temperature.

\subsubsection{Selection of $\mathrm{pH}$ modifiers}

Sodium carbonate and glycine were selected as $\mathrm{pH}$ modifiers. Sodium carbonate represents category of strong alkaline salts and glycine represents category of weakly alkaline salts.

As discussed earlier, carbopol is an anionic polymer with $\mathrm{pH}$ dependent gelling properties. In acidic $\mathrm{pH}$ (stomach) carbopol does not gel and in basic $\mathrm{pH}$ (intestine) carbopol forms strong gel. Since it does not gel, carbopol based matrix burst releases drug in acidic media and as it forms strong gel, carbopol based matrix releases drug incompletely in basic media. Thus, typically a burst release is observed following slow drug release in standard testing conditions (first $2 \mathrm{~h}$ in $\mathrm{pH} 1.2$ and remaining time in $\mathrm{pH}$ 6.8). It was hypothesized that $\mathrm{pH}$ independent carbopol matrix can be developed by incorporating alkaline salts in the formulation. For comparative purposes, a strong alkaline salt e.g. Sodium carbonate and a weak alkaline salt e.g. glycine was selected to test this hypothesis. Following changes are expected in the matrix properties and drug release kinetics (Table 3.1).

i. In acidic media, carbopol is likely to gel in presence of an alkaline salt. Normally, carbopol does not gel in acidic media and allows acid influx into the tablet. As acid penetrates the tablet, it reacts with the alkaline salt. This initiates acid-base reaction and result in increase in micro environment $\mathrm{pH}$. If the increase in the $\mathrm{pH}$ of micro environment is high enough, 
Table 3.1. Comparison of changes in gelling and release characteristics

\begin{tabular}{ccc}
\hline Media & No $\mathrm{pH}$ Modifier & With $\mathrm{pH}$ modifer \\
\hline Acid & No gelling & Surface gelling \\
$(\mathrm{pH}:$ 1.2) & Burst release & No Burst release \\
Basic media & High gel stength & Low gel stength \\
$(\mathrm{pH}:$ 6.8) & Incomplete drug release & Complete drug release \\
\hline
\end{tabular}


then carbopol gels and reduce the drug efflux from the tablet. This will control the burst release.

ii. In basic media, presence of the salt in the tablet further increases micro environment $\mathrm{pH}$. For example, when the $\mathrm{pH}$ of the dissolution media is 6.8 externally, inside the tablet the $\mathrm{pH}$ will be higher than 8 in presence of alkaline salt in the tablet. Carbopol gels have unique $\mathrm{pH}$ dependent strength. Gel strength of carbopol is at peak when the $\mathrm{pH}$ of the media is in the range of 5 to 9 . $\mathrm{pH}$ values lower than 5 and $\mathrm{pH}$ values above 9 will reduce the gel strength. According to this, $\mathrm{pH}$ dependent gel properties, in presence of alkaline salts, carbopol matrix will de-aggregate in basic media ( $\mathrm{pH}$ 6.8). This will help the matrix to achieve complete drug release.

Formulation development of controlled release propranolol hydrochloride tablets was carried out in two steps.

i. Selection of filler and binder for the formulation development

ii. Development of sustained release formulations of propranolol hydrochloride
a. Effect of alkali salts on the drug release
b. Assessment of release mechanism
c. Optimization of release profiles

\subsection{MATERIALS AND METHODS}

\subsubsection{Materials}

Propranolol Hydrochloride, Glyceryl Mono Stearate (GMS) (Stepan, Northfield, IL), Gelucire 50/13 (Gattefosse, Paramus, NJ), Dicalcium phosphate Dihydrate (Ditab®) (Rhodia, 
Cranbury, NJ), Lactose monohydrate spray dried (Foremost, Baraboo, WI), Carbopol 971P

(Noveon, Cleveland, OH), Carbopol 974P (Noveon, Cleveland, OH), Sodium carbonate

anhydrous (Fisher Chemicals, St. Louis, MO)., Glycine (Fisher Chemicals, St. Louis, MO).

\subsubsection{Methods}

\subsubsection{Preparation of granules}

Granules for filler evaluation and preparation of sustained release tablets were prepared using hot melt granulation technique. Ingredients of formulations and composition of the formulations for selection of fillers are given in Table 3.2 and Table 3.3 respectively. Batch size of each formulation was $300 \mathrm{gms}$. Ingredients of the formulations except the waxy binder were blended in Robot Coupe ${ }^{\circledR}$ high shear granulator for 2 min at $1500 \mathrm{rpm}$.

A circulating water batch was attached to jacketed walls of the granulator. Enough time was allowed for the temperature of the granulator to rise to $60^{\circ} \mathrm{C}$ and the temperature of the granulator was maintained at $60^{\circ} \mathrm{C}$ and the blade speed was $1500 \mathrm{rpm}$ for the entire granulation

process. Once the temperature of the granulator reached $60^{\circ} \mathrm{C}$, the waxy binder was added to the granulator and the granulation was carried out for 2 minutes. After 2 minutes, the granulated mass was passed through \# 16 sieve and the obtained granules were allowed to cool to room temperature. Granules were stored in double zip-lock ${ }^{\circledR}$ bags till compression.

\subsubsection{Preparation of tablets}

Granules, as prepared above, were compressed into tablets using rotary tablet punching machine. All batches were compressed with only one punch out of 18 punches of the machine. 
Table 3.2. Ingredients of formulations for selection of fillers

\begin{tabular}{cccc}
\hline Batch code & Wax & Filler & Carbopol \\
\hline FIL -1 & GMS & Lactose & No \\
FIL -2 & GMS & Ditab & No \\
FIL -3 & GMS & Lactose & Yes \\
FIL -4 & GMS & Ditab & Yes \\
FIL -5 & Gelucire 50/13 & Lactose & No \\
FIL -6 & Gelucire 50/13 & Ditab & No \\
FIL -7 & Gelucire 50/13 & Lactose & Yes \\
FIL -8 & Gelucire 50/13 & Ditab & Yes \\
\hline
\end{tabular}


Table 3.3. Composition of formulations for selection of fillers

\begin{tabular}{cccc}
\hline Batch code & Wax $(\%)$ & Filler $(\%)$ & Carbopol $(\%)$ \\
\hline FIL -1 & 10 & 90 & 0 \\
FIL -2 & 10 & 90 & 0 \\
FIL -3 & 10 & 80 & 10 \\
FIL -4 & 10 & 80 & 10 \\
FIL -5 & 10 & 90 & 0 \\
FIL -6 & 10 & 90 & 0 \\
FIL -7 & 10 & 80 & 10 \\
FIL -8 & 10 & 80 & 10 \\
\hline
\end{tabular}


Tablet weight of the all formulations was $320 \mathrm{mg}$ and tablets were prepared using 5/16" deepconcave punches. Tablet punching machine was operated with only one punch and remaining seventeen die cavities of the rotary punching machine were covered with blank dies. Machine was operated at 20 RPM for preparation of all formulations in the study.

\subsubsection{Bulk density evaluation}

Bulk density of the granules was evaluated using a $50 \mathrm{ml}$ measuring cylinder and a balance. Initially the weight of the measuring cylinder was tarred. Then, the granules were poured into the measuring cylinder till the fill volume was $40 \mathrm{ml}$ using a funnel. Then the weight of the measuring cylinder with granules was taken. This gives the weight of the granules. Bulk density of the granules was calculated using following formula and the bulk density of formulations is given in Table 3.4.

Bulk density $=$ Weight of granules/Volume of granules

\subsubsection{Evaluation of dynamic flow rate}

Dynamic flow of the material was determined using a Hanson Flowdex ${ }^{\circledR}$ (Hanson Research Corporation, Chatsworth, CA). The device consists of a metal cylinder attached to a metal plate with an orifice at the bottom. This orifice was closed or opened by the movable stage attached to the bottom of the plate. Material was filled into the cylinder with the orifice closed. Approximately three fourth of cylinder was filled with the material. Orifice was selected based

on final dosage form weight. Granules were to be compressed as $100 \mathrm{mg}$ tablets for evaluation of compressibility characteristics. Therefore 8 millimeter orifice was selected. 
Table 3.4. Physical characteristics of formulations for filler selection $\uparrow$

\begin{tabular}{ccccc}
\hline Batch code & Bulk density $(\mathrm{gms} / \mathrm{ml})$ & $\begin{array}{c}\text { Flow rate } \\
(\mathrm{gms} / \mathrm{sec})\end{array}$ & $\begin{array}{c}\text { Tablet hardness } \\
\text { at } 0.5 \mathrm{mT}(\mathrm{kps})\end{array}$ & $\begin{array}{c}\text { Tablet hardness } \\
\text { at } 1 \mathrm{mT}(\mathrm{kps})\end{array}$ \\
\hline FIL -1 & $0.529 \pm 0.02$ & $15.98 \pm 0.51$ & $2.32 \pm 0.33$ & $2.38 \pm 0.16$ \\
FIL -2 & $0.865 \pm 0.04$ & $26.14 \pm 0.78$ & $3.35 \pm 0.15$ & $3.60 \pm 0.11$ \\
FIL -3 & $0.561 \pm 0.02$ & $25.33 \pm 0.81$ & $2.98 \pm 0.13$ & $3.30 \pm 0.11$ \\
FIL -4 & $1.136 \pm 0.02$ & $44.29 \pm 1.25$ & $4.97 \pm 0.14$ & $5.27 \pm 0.69$ \\
FIL -5 & $0.531 \pm 0.02$ & $10.54 \pm 0.56$ & $1.98 \pm 0.23$ & $2.13 \pm 0.08$ \\
FIL -6 & $0.917 \pm 0.05$ & $23.54 \pm 0.67$ & $4.23 \pm 0.29$ & $4.37 \pm 0.32$ \\
FIL -7 & $0.503 \pm 0.03$ & $0.00 \pm 0.00$ & $3.97 \pm 0.40$ & $4.40 \pm 0.60$ \\
FIL - 8 & $1.011 \pm 0.03$ & $19.87 \pm 0.78$ & $3.47 \pm 0.42$ & $3.72 \pm 0.52$ \\
\hline
\end{tabular}

$\dagger$ indicates values presented in the table are average of three measurements. Average values are presented with standard deviation of three measurements. 
Once filled, the movable stage blocking the orifice was removed from the bottom of the plate and the material was allowed to flow through the orifice onto a balance, which was connected to a computer. The dynamic weight change of material that flows through the orifice

and time in millisecond were recorded electronically using Software WinWedge ${ }^{\circledR}$ (TAL Technologies, Inc., Philadelphia, PA). The data obtained was plotted as the weight of the material versus time. The flow rate was then determined as the slope of the linear regression of the plot and the dynamic flow rate of formulations for filler selection is given in Table 3.4.

\subsubsection{Evaluation of compressibility}

Compressibility of the granules was evaluated by estimating tablet hardness of tablets prepared from the granules. Granules, as prepared above, were compressed into tablets using rotary tablet punching machine. Tablet weight of the all formulations was $100 \mathrm{mg}$ and tablets were prepared using $1 / 4$ " flat faced punches. Tablet punching machine was operated with only one punch and remaining seventeen die cavities of the rotary punching machine were covered with blank dies. Machine was operated at 20 RPM for compressibility evaluation study. Tablets were compressed in two compression forces i.e., $0.5 \mathrm{mT}$ and $1 \mathrm{mT}$. After compression the tablets were stored in Ziplock ${ }^{\circledR}$ bags for one day before hardness evaluation. Tablet hardness was tested for randomly selected six tablets for each compression force. Hardness testing was done using Pharmatest ${ }^{\circledR}$ hardness tester and the hardness of tablets prepared at $0.5 \mathrm{mT}$ and $1 \mathrm{mT}$ compression force is given in Table 3.4. 


\subsubsection{Evaluation of in vitro drug release}

In vitro drug release characteristics of tablets were assessed using Paddle method (USP method II). Dissolution test was carried out in two different $\mathrm{pH}$ media to simulation gastro intestinal conditions. First $2 \mathrm{~h}$, the dissolution test was carried out in $750 \mathrm{ml}$ of pH 1.2 acidic media $(0.1 \mathrm{~N} \mathrm{HCl})$. Then, $250 \mathrm{ml}$ of $1 \mathrm{~N}$ Tribasic sodium phosphate solution was added to increase the $\mathrm{pH}$ of the dissolution media to 6.8. Required quantity of $1 \mathrm{~N}$ Sodium hydroxide solution was used to adjust the $\mathrm{pH}$ of the dissolution media if necessary after addition of $250 \mathrm{ml}$ of $1 \mathrm{~N}$ tribasic sodium phosphate solution. Thus, dissolution was carried out in $250 \mathrm{ml}$ of $0.1 \mathrm{~N}$ $\mathrm{HCl}$ for first $2 \mathrm{~h}$, then $1000 \mathrm{ml}$ of combination media with $\mathrm{pH} 6.8$ for remaining $10 \mathrm{~h}$. Total length of the dissolution test was $12 \mathrm{~h}$.

Drug release was measured using fiber optic UV probes with $5 \mathrm{~mm}$ inserts. Inserts are fixed at one end of the UV probe and the other end of the UV probe is connected to UV spectrophotometer with photo diode array detector. One end of the UV probe containing insert was immersed into the dissolution media during the dissolution study. Insert contains a reflecting mirror and the UV probe is designed in such a way that presence of insert forms an open chamber in which the drug solution will flow through during dissolution test. During dissolution test, at predetermined time intervals, the UV light from UV spectrophotometer is passed through UV probe. The UV light travels in the UV probe and transmits through the drug solution and reflected back to the detector by the reflecting surface in the insert. Based on the drug concentration in the solution contained in the open chamber, there are differences in the incident light and reflected light. UV absorbance of the drug solution was calculated from the differences in the intensity of incident light and reflected light. Reference drug absorbance was used to calculate the drug concentration in the solution. Calculation of drug concentration was based on 
reference drug absorption taken at maximum wavelength of absorption for propranolol hydrochloride i.e., $278 \mathrm{~nm}$. Volume of the dissolution media at the sampling time, drug loading were used to calculate the percent drug released. All these calculations were done in Indigo ${ }^{\circledR}$ software and the results were exported to Microsoft Excel ${ }^{\circledR}$.

\subsubsection{Evaluation of mechanism of drug release}

The formulations' capacity for hydration (buffer medium uptake) and their extent of erosion were evaluated gravimetrically. This study was carried out similar to in vitro dissolution test i.e., for first $2 \mathrm{~h}$ the medium uptake was carried out in $0.1 \mathrm{~N} \mathrm{HCl}$ then in $1000 \mathrm{ml}$ of combination media containing $750 \mathrm{ml}$ of $0.1 \mathrm{~N} \mathrm{HCl}$ and $250 \mathrm{ml}$ of $1 \mathrm{~N}$ tribasic sodium phosphate solution. For each time point, two tablets of each formulation were weighed individually and exposed to dissolution media. The test conditions such as paddle speed and the temperature were similar to that of dissolution test. At specific time points, tablets were removed from the medium, patted gently with a tissue paper, weighed, dried at $60^{\circ} \mathrm{C}$ until constant weight was achieved. Percent weight gain (hydration) and \% mass loss (erosion) were calculated according to the equations 3.1 and 3.2 using original, wet, and dry weight values obtained from the testing.

$$
\begin{aligned}
& \% \text { weight gain }=\frac{\text { Wetweight }- \text { Dryweight }}{\text { Dryweight }} \times 100 \\
& \% \text { erosion }=\frac{\text { Originalweight }- \text { remaining }(\text { dry }) \text { weight }}{\text { Originalweight }} \times 100
\end{aligned}
$$




\subsection{RESULTS AND DISCUSSION}

\subsubsection{Selection of fillers}

\subsubsection{Bulk density}

Statistical analysis of effects of carbopol, type of wax and type of filler is given in Table 3.5. Analysis indicates that change in wax type from Glyceryl Mono Stearate to Gelucire 50/13 significantly reduced the bulk density of the granules obtained in the hot melt granulation. Changing filler in the formulation from lactose to Ditab significantly increased the bulk density of the granules. This could be attributed to the higher density of Ditab compared to that of lactose. Addition of carbopol in the formulations significantly increased the bulk density of the granules. This implies that carbopol can act as binder and enhance the densification of granules during hot melt granulation. This results in formation of higher density granules in presence of carbopol.

No significant interaction was observed between effects of wax and fillers on the granule bulk density. This implies that effect of wax on the bulk densities of granules remains unchanged in lactose and Ditab. However, there is significant interaction observed between effects of wax and presence of carbopol in the formulation. This indicates, when Gelucire 50/13 was used as waxy binder, the increase in the granule bulk density is marginal. However, when Glyeryl Mono Stearate was used as waxy binder the increase in the granule bulk density is significant. If bulk densities of lactose and Ditab are compared, lactose has less density than Ditab. As densities of pure excipients are significantly different, the granules obtained from them will have significantly different bulk densities. 
Table 3.5. Statistical analysis of physical characteristics

\begin{tabular}{ccccc}
\hline Effect & $\begin{array}{c}\text { Bulk density } \\
(\mathrm{gms} / \mathrm{sec})\end{array}$ & $\begin{array}{c}\text { Flow rate } \\
(\mathrm{gms} / \mathrm{sec})\end{array}$ & $\begin{array}{c}\text { Tablet hardness } \\
\text { at } 0.4 \mathrm{mT}(\mathrm{kps})\end{array}$ & $\begin{array}{c}\text { Tablet hardness } \\
\text { at 1 mT }(\mathrm{kps})\end{array}$ \\
\hline Intercept & $0.76^{*}$ & $20.71^{*}$ & $3.41^{*}$ & $3.65^{*}$ \\
Wax (GMS - Gelucire) & $0.02^{*}$ & $7.22^{*}$ & 0.00 & -0.01 \\
Filler (Lactose - Ditab) & $-0.23^{*}$ & $-7.75^{*}$ & $-0.60^{*}$ & $-0.59^{*}$ \\
Carbopol (Yes-No) & $-0.05^{*}$ & $-1.66^{*}$ & $-0.44^{*}$ & $-0.53^{*}$ \\
Wax X Filler & 0.00 & $0.47^{*}$ & -0.16 & -0.20 \\
Wax X Carbopol & $-0.03^{*}$ & $-5.21^{*}$ & -0.13 & -0.12 \\
Filler X Carbopol & $0.05^{*}$ & $1.96^{*}$ & -0.23 & -0.27 \\
\hline
\end{tabular}

* indicates values are significant at $\mathrm{P}<0.05$. 
When lactose was used as filler in the formulations, addition of carbopol to the formulation had no significant influence on the granule bulk density. However, when Ditab was used as filler in the formulation, addition of carbopol in the formulation significantly increased the granule bulk density.

\subsubsection{Dynamic flow rate}

Statistical analysis of effect of carbopol, type of wax and type of filler on flow rate is given in Table 3.5. Statistical analysis indicates that change of wax type from Glyeryl Mono Stearate to Gelucire 50/13 significantly reduced the flow rate. Changing filler type from lactose to Ditab significantly increased the flow rate of the granules. Presence of carbopol in the formulation increased the flow rate significantly. Significant interaction existed between the effect of wax type and the presence of carbopol in the formulation. This indicates, when no carbopol was present in the formulation, change in wax type from Glyceryl Mono Stearate to Gelucire marginally changed the flow rate. However, when carbopol was present in the formulation, change in the waxy binder from Glyceryl Mono Stearate to Gelucire significantly reduced the flow rate. This confirms that effect of waxy binder on the granule flow rate is dependent on the presence of carbopol. Similarly, effect of filler on the flow rate of the granule is dependent on the presence of carbopol in the formulation.

When lactose is used as filler, addition of carbopol did not significantly increase flow rate. However, when Ditab was used as filler, addition of carbopol significantly increased the flow rate. Thus, the effect of carbopol on the granule flow rate is dependent on the type of filler used in the formulation. 


\subsubsection{Compressibility}

Statistical analysis of the effect of Carbopol, type of wax and type of filler on the tablet hardness at low compression force $(0.4 \mathrm{mT})$ is given in the Table 3.5. Statistical analysis indicates that change in the wax type had no significant influence on the tablet hardness at lower compression force. However, type of filler and presence of carbopol had significant influence on the tablet hardness. Change of filler from lactose to Ditab significantly increased the tablet hardness. Similarly, addition of carbopol in the formulation significantly increased the tablet hardness. Surprisingly, none of the interactions were significant. This implies, the main effects of individual factors remain unchanged in presence of other factors.

Statistical analysis of the effect of carbopol, type of wax and type of filler on the tablet hardness at high compression force $(1 \mathrm{mT})$ is given in Table 3.5. Statistical analysis indicates that the effect of wax type, filler type, presence of carbopol had similar influence on the tablet hardness (at high compression pressure) as their influence on tablet hardness at low compression pressure. That is, change of filler to Ditab in the formulation, increased the tablet hardness and addition of carbopol in the formulation increased the tablet hardness. None of the interactions among these factors were significant.

It is noteworthy to mention, that the maximum hardness of each formulation was reached at low compression force and only marginal increase in the tablet hardness was observed when tablets were prepared at high compression pressure.

In summary, fillers and binders were evaluated for their ability to flow well (at least 25 gms/sec) and compress well (4 kps hardness). Based on the results, Dicalcium phosphate is selected as filler and Glyceryl Mono Stearate as waxy binder for formulation development. 


\subsubsection{Development of controlled release formulation}

\subsubsection{Formulations containing sodium carbonate as release modifer}

Ingredients and composition of formulations for product development are presented in Table 3.6 and Table 3.7 respectively. Formulations PHP 1 to PHP 3 served as controls to evaluate the effect of $\mathrm{pH}$ modifer on the release profiles of formulations containing different concentrations of carbopol. Formulations PHP 4 to PHP 6 contained 10\% sodium carbonate as release modifier. Comparison of the release profiles among different formulations was made by comparing the mean values of the drug release at $1 \mathrm{~h}, 3 \mathrm{~h}, 6 \mathrm{~h}$ and $12 \mathrm{~h}$. These time points were selected as USP standard for sustained release dosage form of Propanolol Hydrochloride containing $80 \mathrm{mg}$ of the active drug. USP recommends comparison of drug release at these time points. PHP 1 and PHP 2 formulations served as controls to estimate the effect of Carbopol on the drug release. PHP 1 contained all ingredients except carbopol and $\mathrm{pH}$ modifer. PHP 2 and PHP 3 contained all ingredients except pH modifer. Release profiles of PHP 1, PHP 2 and PHP 3 are presented in Figure 3.1. Student t-test comparing the amount of drug released between these two formulations was found to be significant at $1 \mathrm{~h}, 3 \mathrm{~h}, 6 \mathrm{~h}$ and $12 \mathrm{~h}$. This implies, addition of carbopol can significantly reduce the drug release and the effect of carbopol on the drug release is dependent on the carbopol concentration. That is, formulations contain low levels of carbopol release drug faster than formulations containing high levels of carbopol. Formulations PHP 4 and PHP 5 show the effect of $10 \%$ of sodium carbonate on the drug release profiles of formulations containing $10 \%$ and $5 \%$ of carbopol respectively.

Comparison of dissolution profiles formulations containing carbopol and carbopol with sodium carbonate is presented in Figure 3.2. 
Table 3.6. Ingredients of formulations for product development

\begin{tabular}{|c|c|c|c|c|}
\hline Batch code & Carbopol type & Wax & $\mathrm{pH}$ modifer & Filler \\
\hline PHP - 1 & No carbopol & GMS & No $\mathrm{pH}$ modifer & Ditab \\
\hline PHP - 2 & $971 \mathrm{P}$ & GMS & No $\mathrm{pH}$ modifer & Ditab \\
\hline PHP - 3 & $971 \mathrm{P}$ & GMS & No $\mathrm{pH}$ modifer & Ditab \\
\hline PHP - 4 & $971 \mathrm{P}$ & GMS & $\begin{array}{l}\text { Sodium } \\
\text { carbonate }\end{array}$ & Ditab \\
\hline PHP - 5 & $971 \mathrm{P}$ & GMS & $\begin{array}{l}\text { Sodium } \\
\text { carbonate }\end{array}$ & Ditab \\
\hline PHP - 6 & No carbopol & GMS & $\begin{array}{c}\text { Sodium } \\
\text { carbonate }\end{array}$ & Ditab \\
\hline PHP - 7 & $971 \mathrm{P}$ & GMS & Glycine & Ditab \\
\hline PHP - 8 & $971 \mathrm{P}$ & GMS & Glycine & Ditab \\
\hline PHP - 9 & No carbopol & GMS & Glycine & Ditab \\
\hline PHP - 10 & $971 \mathrm{P}$ & GMS & No $\mathrm{pH}$ modifer & Lactose \\
\hline PHP - 11 & $974 \mathrm{P}$ & GMS & No $\mathrm{pH}$ modifer & Lactose \\
\hline PHP - 12 & $974 \mathrm{P}$ & GMS & No $\mathrm{pH}$ modifer & Ditab \\
\hline
\end{tabular}


Table 3.7. Composition of formulations for product development

\begin{tabular}{ccccc}
\hline Batch code & Carbopol (\%) & Wax $(\%)$ & $\mathrm{pH}$ modifer $(\%)$ & Filler $(\%)$ \\
\hline PHP - & 0 & 10 & 0 & 65 \\
PHP - & 10 & 10 & 0 & 55 \\
PHP - 3 & 5 & 10 & 0 & 60 \\
PHP - 4 & 10 & 10 & 10 & 45 \\
PHP - 5 & 5 & 10 & 10 & 50 \\
PHP -6 & 0 & 10 & 10 & 55 \\
PHP - 7 & 10 & 10 & 10 & 45 \\
PHP - 8 & 5 & 10 & 10 & 50 \\
PHP - 9 & 0 & 10 & 10 & 55 \\
PHP - 10 & 10 & 10 & 0 & 55 \\
PHP - 11 & 10 & 10 & 0 & 55 \\
PHP - 12 & 10 & 10 & 0 & 55 \\
\hline
\end{tabular}




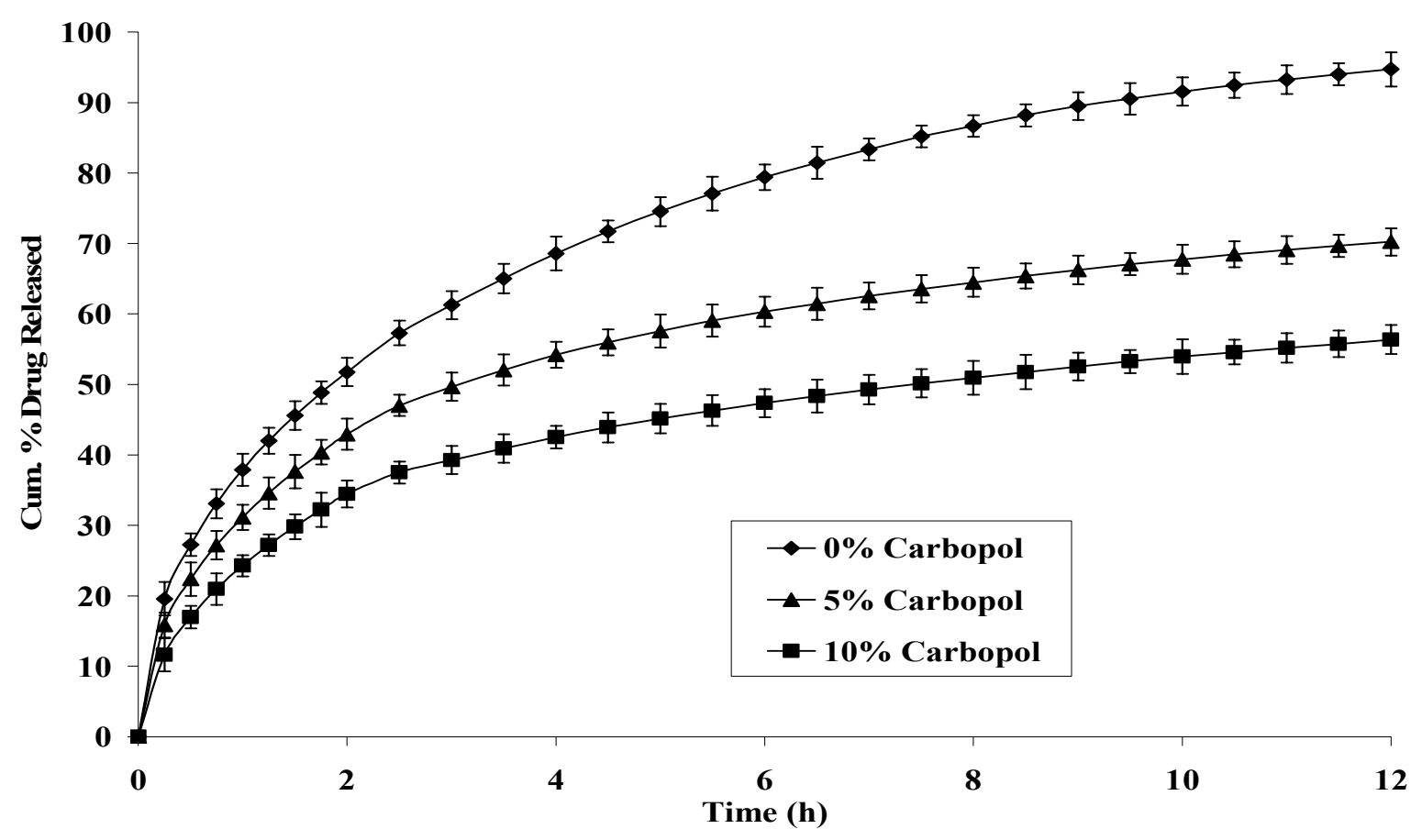

Figure 3.1. Effect of carbopol on drug release $\uparrow$

$\uparrow$ Each data point represents average of three measurements. Standard deviation of three measurements is presented as error bars. 


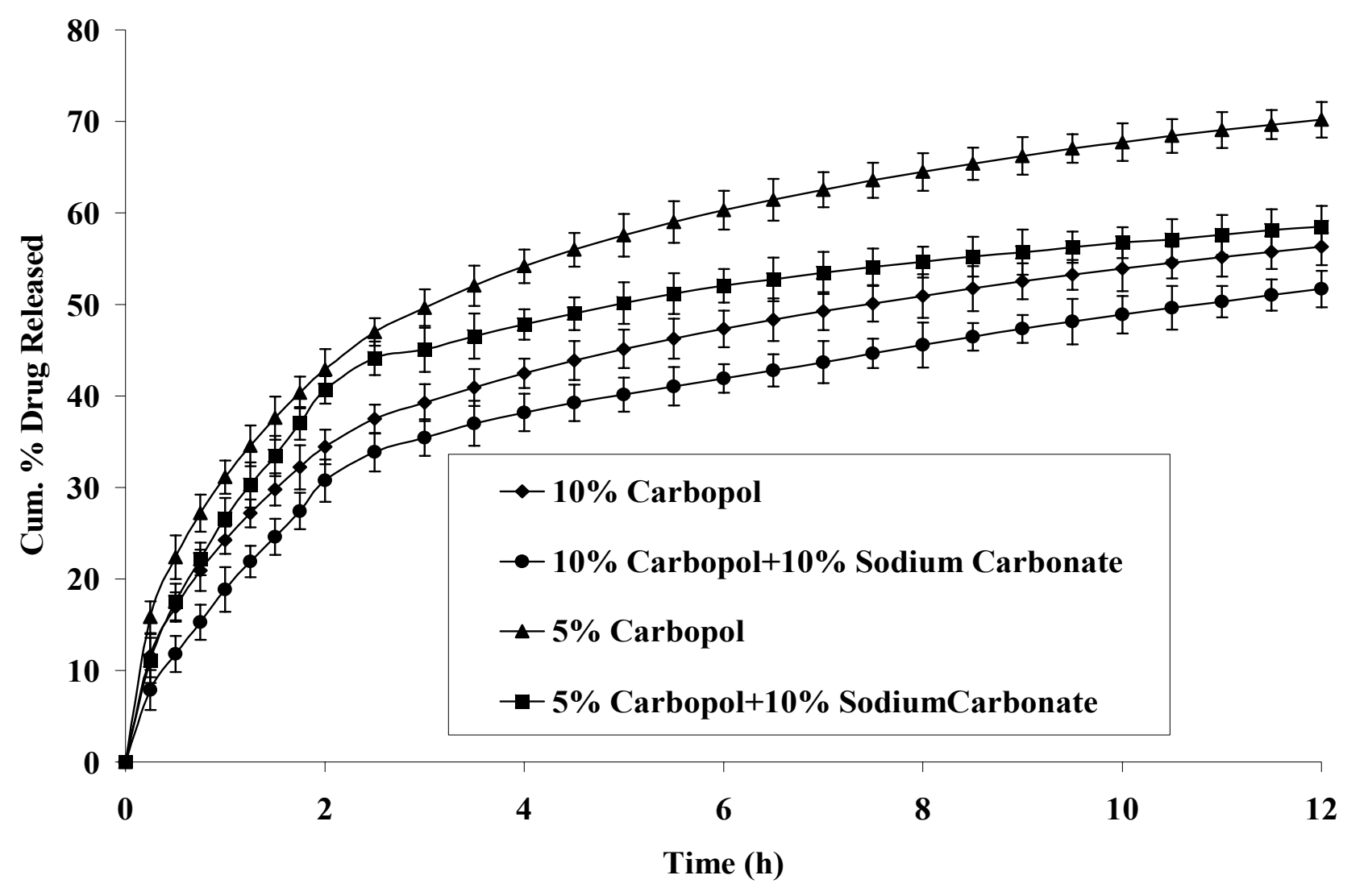

Figure 3.2. Effect of $10 \%$ sodium carbonate on dissolution profiles $\uparrow$

$\dagger$ Each data point represents average of three measurements. Standard deviation of three measurements is presented as error bars. 
If formulations PHP 2 and PHP 4 are compared, then the effect of adding 10\% sodium carbonate to formulations containing $10 \%$ carbopol will be evident. Student t-test comparison of drug released at $1 \mathrm{~h}, 3 \mathrm{~h}, 6 \mathrm{~h}$ and $12 \mathrm{~h}$ revealed that the drug release was significantly different in these formulations at $1 \mathrm{~h}, 6 \mathrm{~h}$ and $12 \mathrm{~h}$. Formulations containing sodium carbonate had less extent of drug release. This implies, that sodium carbonate can reduce the drug release from matrix tablets containing carbopol. A similar trend was observed in comparison between formulations PHP 3 and PHP 6. This implies that Sodium carbonate can reduce the drug release from matrix tablets containing carbopol irrespective of carbopol's concentration. However, the extent of effect of sodium carbonate on drug release is dependent on carbopol's concentration. This implies that carbopol has more influence on the drug release than the sodium carbonate. Thus, sodium carbonate must always be used in conjunction with carbopol to effectively control the drug release. This fact is evident in dissolution profiles of formulation PHP 6. When compared against PHP 1, PHP 6 had no significant difference in the drug release at all data points. This implies, having only sodium carbonate in the tablets with no carbopol is an ineffective way of controlling the drug release. It is noteworthy to mention that addition of sodium carbonate has significantly reduced the drug release throughout the entire dissolution profile. This is in contrary to the expected outcome of the presence of sodium carbonate in the tablets. It was expected that when sodium carbonate is present in the tablet and the tablet is in dissolution media with $\mathrm{pH} 6.8$, then the microenvironment $\mathrm{pH}$ within the tablet will be higher than $\mathrm{pH}$ 9. This will degenerate the matrix and release the drug faster.

However, the results were different than expected. This implies that drug-carbopol complex formed at basic $\mathrm{pH}$ value is predominantly controlling the drug release. Moreover, in comparison among PHP1, PHP2 and PHP 3, it is evident that formulations containing carbopol 
had incomplete drug release. This implies the formation of carbopol - propranolol hydrochloride complex results in incomplete drug release.

\subsubsection{Formulations containing glycine as release modifer}

Formulations PHP 7 to PHP 9 contained glycine as release modifier. Comparison of the release profiles among different formulations were made by comparing the mean values of the drug release at $1 \mathrm{~h}, 3 \mathrm{~h}, 6 \mathrm{~h}$ and $12 \mathrm{~h}$. Formulations PHP 7 and PHP 8 shows the effect of $10 \%$ of glycine on the drug release profiles of tablets containing $10 \%$ and $5 \%$ of carbopol respectively. Dissolution profiles of PHP 7 and PHP 8 with their control formulations containing similar concentration of carbopol are presented in Figure 3.3. If formulations PHP 2 and PHP 7 are compared, then the effect of adding $10 \%$ glycine to formulations containing $10 \%$ carbopol will be evident. Student t-test comparison of drug released at $1 \mathrm{~h}, 3 \mathrm{~h}, 6 \mathrm{~h}$ and $12 \mathrm{~h}$ revealed that the differences in the drug release were statistically insignificant at all aforementioned time points.

This implies that glycine can not reduce the drug release from matrix tablets containing carbopol at $10 \%$ concentration. A similar trend was observed in comparison between formulations PHP 3 and PHP 8. This implies that glycine can not reduce the drug release from matrix tablets containing carbopol and this effect is similar in low (5\%) and high (10\%) carbopol concentration. These comparisons illustrate that glycine is an ineffective release modifier at $10 \%$ concentration.

\subsubsection{Evaluation of mechanism of drug release}

Water absorption and erosion profile of formulations containing no carbopol (control), 10\% carbopol, $10 \%$ carbopol with $10 \%$ sodium carbonate and $10 \%$ carbopol with $10 \%$ glycine are presented in Figure 3.4. 


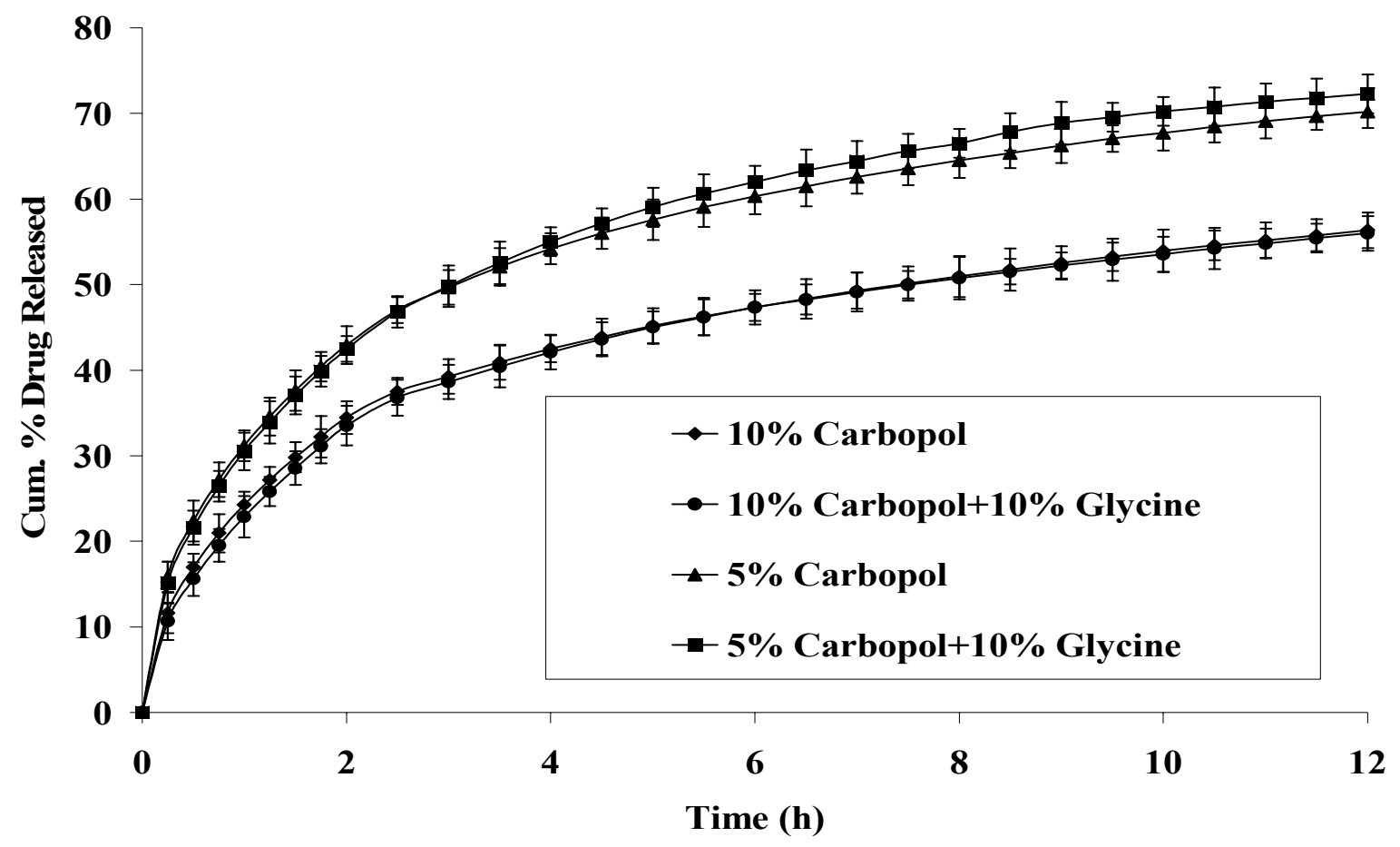

Figure 3.3. Effect of $10 \%$ glycine on dissolution profiles $\uparrow$

$\dagger$ Each data point represents average of three measurements. Standard deviation of three measurements is presented as error bars. 


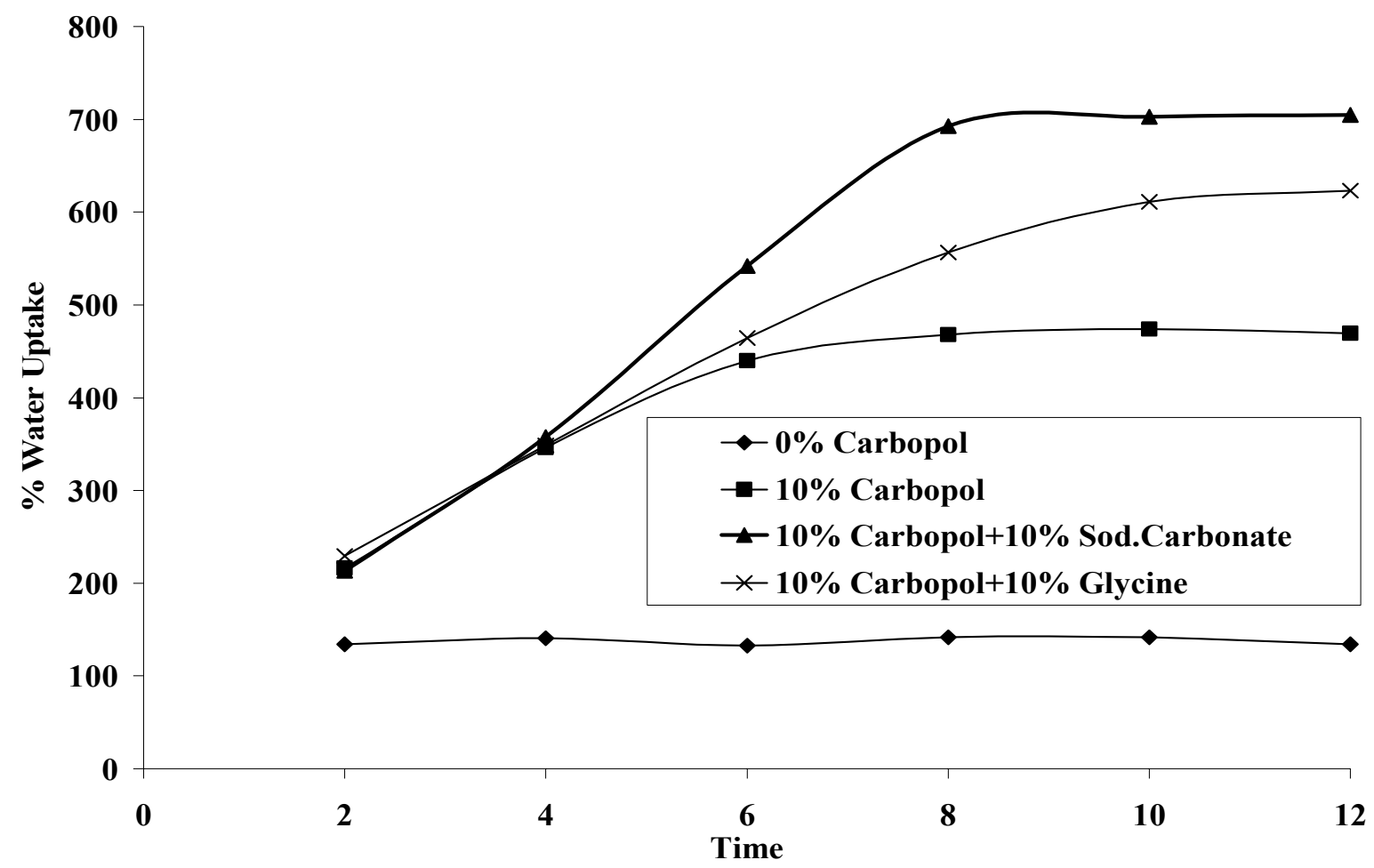

Figure 3.4. Water absorption profile of formulations 
Formulation containing no carbopol had almost constant amount of water in the tablet throughout the testing period. This formulation was made with only waxy binder. Literature reports that formulations made with waxy materials release drug through pore diffusion. Pore diffusion is concentration driven drug diffusion process in which drug is released through pores in the matrix. Pore diffusion is not dependent on the water uptake of the matrix. This is evident from the water absorption profile of tablet prepared with waxy binder.

Formulation containing 10\% carbopol had significant increase in water uptake till $8 \mathrm{~h}$ then the increase was marginal. This can be attributed to the presence of carbopol in the tablet. Carbpol is hydrophilic, swelling polymer. Matrix tablets containing such polymers continue to absorb dissolution media and this absorption depends on gel layer thickness, geometrical extent of swelling, solubility of the matrix components. If there is no interaction between drug and other components of the matrix, then the drug release must chiefly be dependent on the extent of water uptake of the matrix. Formulation containing 10\% carbopol and $10 \%$ sodium carbonate had higher water uptake than formulation containing only carbopol. This could be because of the osmotic gradient created due to the dissolution of sodium carbonate in the tablet and the relaxation of polymeric chains due to presence of a salt that can induce ionization of polymeric chains.

A similar trend in water absorption was observed in formulation containing $10 \%$ carbopol and $10 \%$ glycine, although the extent of water absorption was less than that of formulation containing sodium carbonate.This implies alkalinity of the salt has some relationship with extent of water absorption i.e., strong alkaline salts can induce the higher water absorption of the matrix. Thus, based on the effect of water absorption on the drug release, the water absorption of the matrix system can be modulated by adjusting alkalinity of the salts. Unexpectedly, the extent 
of media absorption at the end of $2 \mathrm{~h}$ remained virtually same in all four formulations.

Supposedly, the formulations containing alkaline salts were expected to have less acid uptake as the matrix is expected to gel due to change in microenvironment $\mathrm{pH}$ inside the tablet.

Erosion profiles of aforementioned formulations are given in Figure 3.5. This Figure 3.5 reveals that the extent of erosion of all formulations was similar. This was unexpected. Formulations containing alkali salts were expected to have higher extent of matrix erosion due to the expected higher microenvironment $\mathrm{pH}$.

Water absorption and erosion studies reveal two important facts. They are: First, alkali concentration used in the formulations was insufficient to reduce the acid uptake into the tablets. Second, alkali concentration used in the formulation was insufficient to induce the matrix erosion in $\mathrm{pH} 6.8$.

Relationship between the \%water uptake and \% erosion with \%cumulative drug released respectively is presented in Figure 3.6 and Figure 3.7. Figure 3.6 showing relationship between $\%$ water uptake and \%drug released reveals a positive slope in formulations containing carbopol and carbopol with alkai salts. This implies the drug release is related to \% water uptake only less extent. Figure 3.7 showing relationship between $\%$ erosion and $\%$ drug released illustrates no relationship between matrix erosion and drug release. These plots confirm that the drug release is related to $\%$ water uptake to less degree and has no relationship with $\%$ erosion. Absence of matrix erosion can be attributed to incomplete drug release in formulations containing carbopol.

Since the drug release is not significantly dependent on the \%water uptake and $\%$ erosion, the main mechanism by which the drug release is controlled is ionic interaction between drug and carbopol.

Thus, combining these results, to achieve complete drug release from carbopol matrix the 


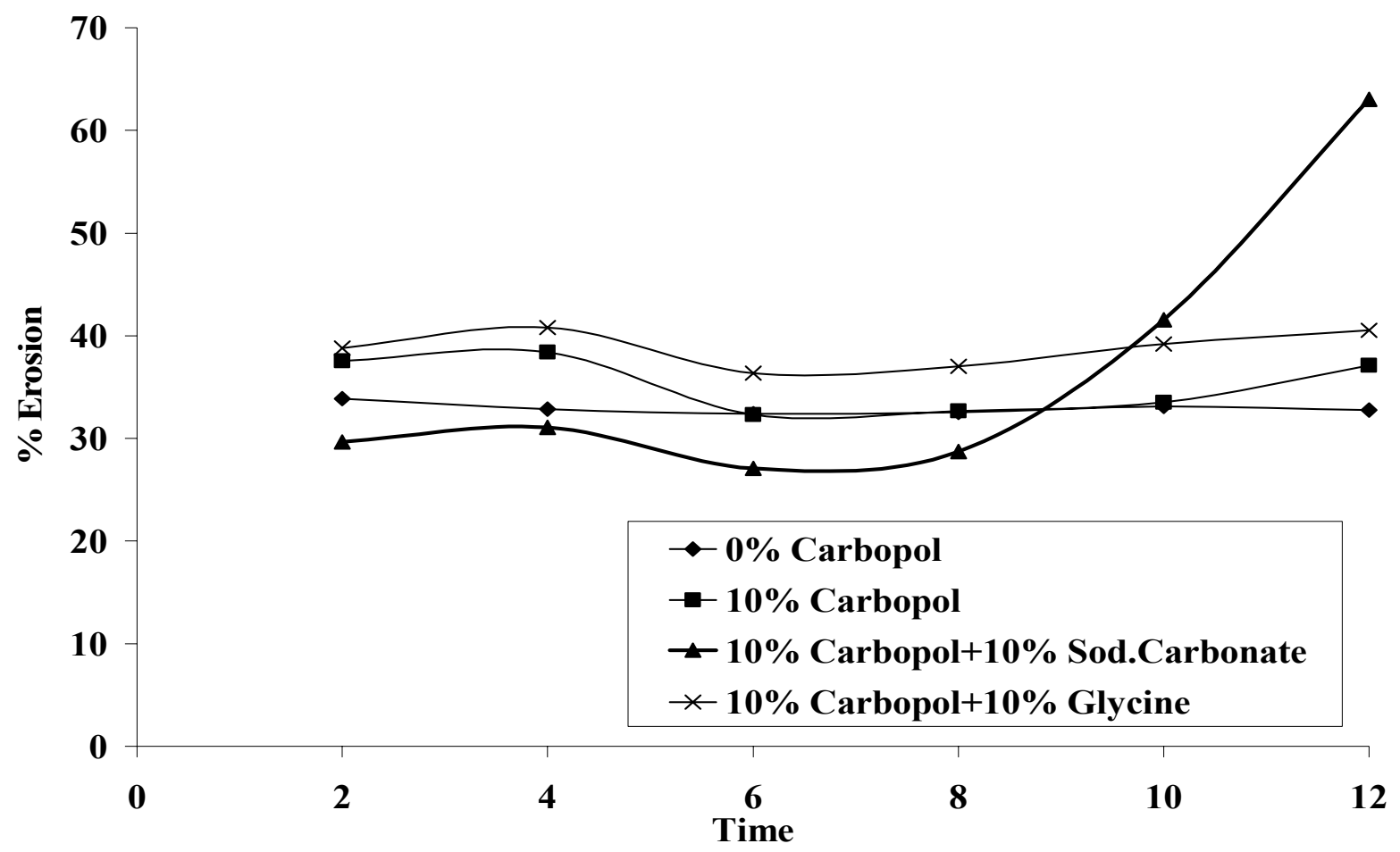

Figure 3.5. Erosion profiles of formulations 


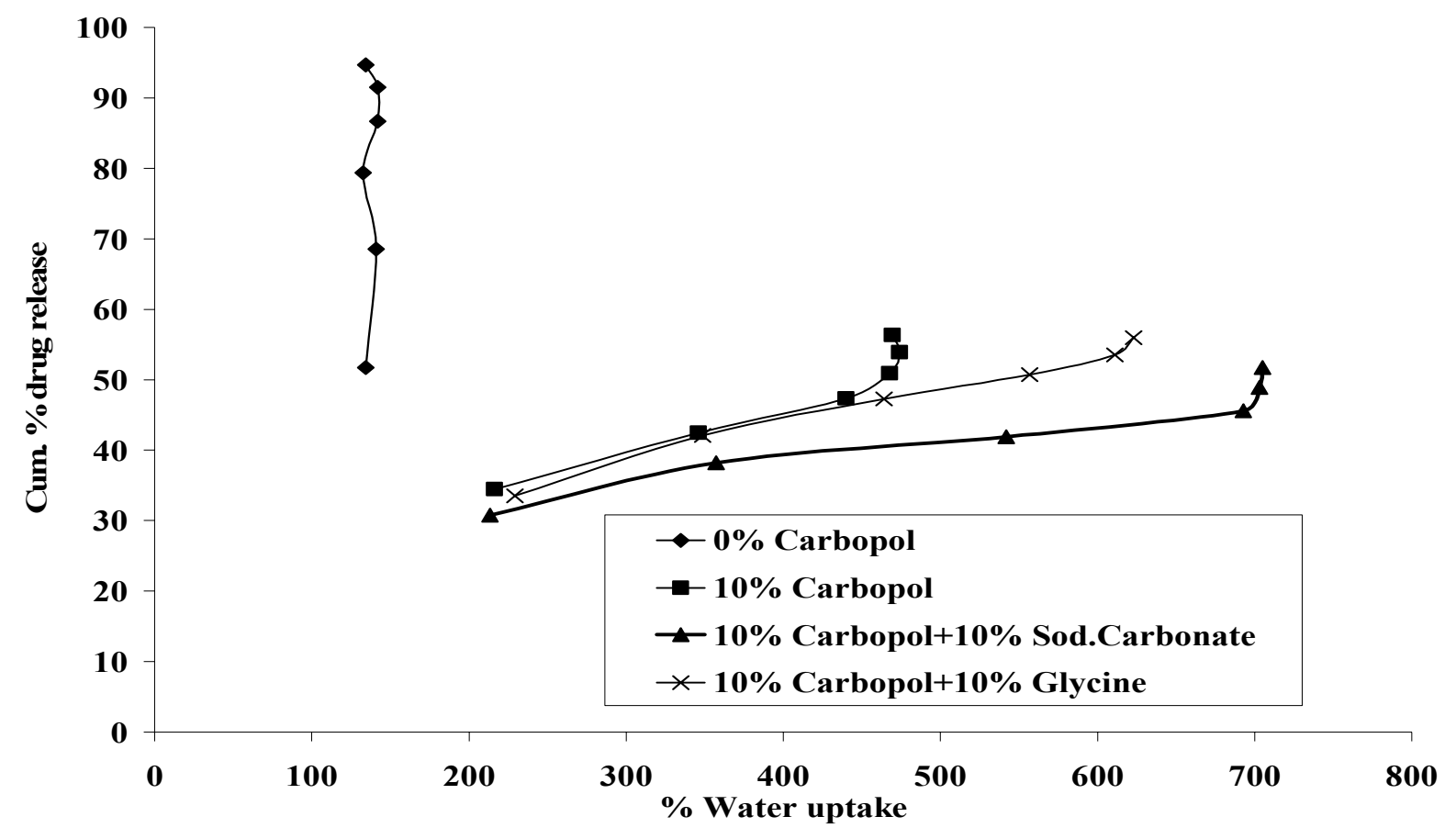

Figure 3.6. Relationship between water absorption and dissolution profiles 


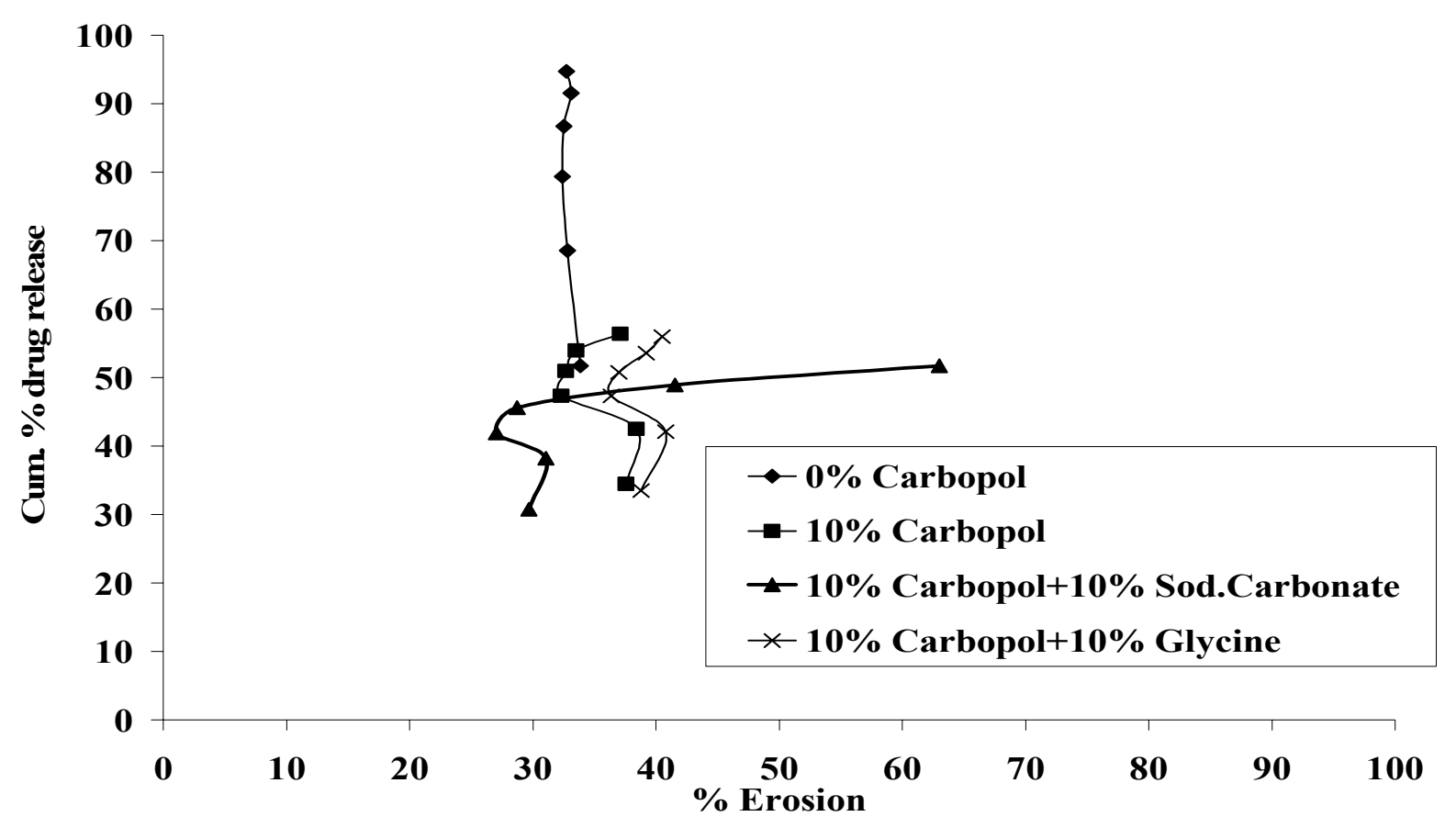

Figure 3.7. Relationship between erosion and dissolution profiles 
grade of carbopol selected must have less chance to form complex with the drug. For example, carbopol 974, a grade with higher crosslink density, may be selected. Excipient in the formulation must support matrix erosion. This will counterbalance the effects of ionic complexation. All aforementioned formulations contained Ditab as filler. Ditab is water insoluble. It can be replaced with Lactose Monohydrate, water soluble filler.

\subsubsection{Formulations with lactose filler}

Ditab, the insoluble filler, was replaced with lactose monohydrate to achieve complete drug release while keeping the acidic drug release in control. Complete drug release can also be achieved by reducing the polymer concentration in the formulation. However, if the polymer concentration is reduced the duration of drug release will be shorter and the drug release in acid will be higher. This is undesirable to develop a formulation that complies with USP standards. Comparison of dissolution profiles of formulations containing Ditab as filler and lactose monohydrate as filler is presented in Figure 3.8. The comparison reveals, change in the filler from Ditab, an insoluble filler, to lactose monohydrate, a soluble filler has increased the drug release. The amount of drug released at $12 \mathrm{~h}$ was $68.43 \%$ in lactose monohydrate based formulations and this is significantly higher compared to $12 \mathrm{~h}$ release of $56.35 \%$ in Ditab based formulations with carbopol 971P as release controlling polymer. However, the USP compliant formulations must have $12 \mathrm{~h}$ drug release of not less than $80 \%$. Thus, change in filler type to filler with higher solubility failed to yield the USP compliant formulation. 


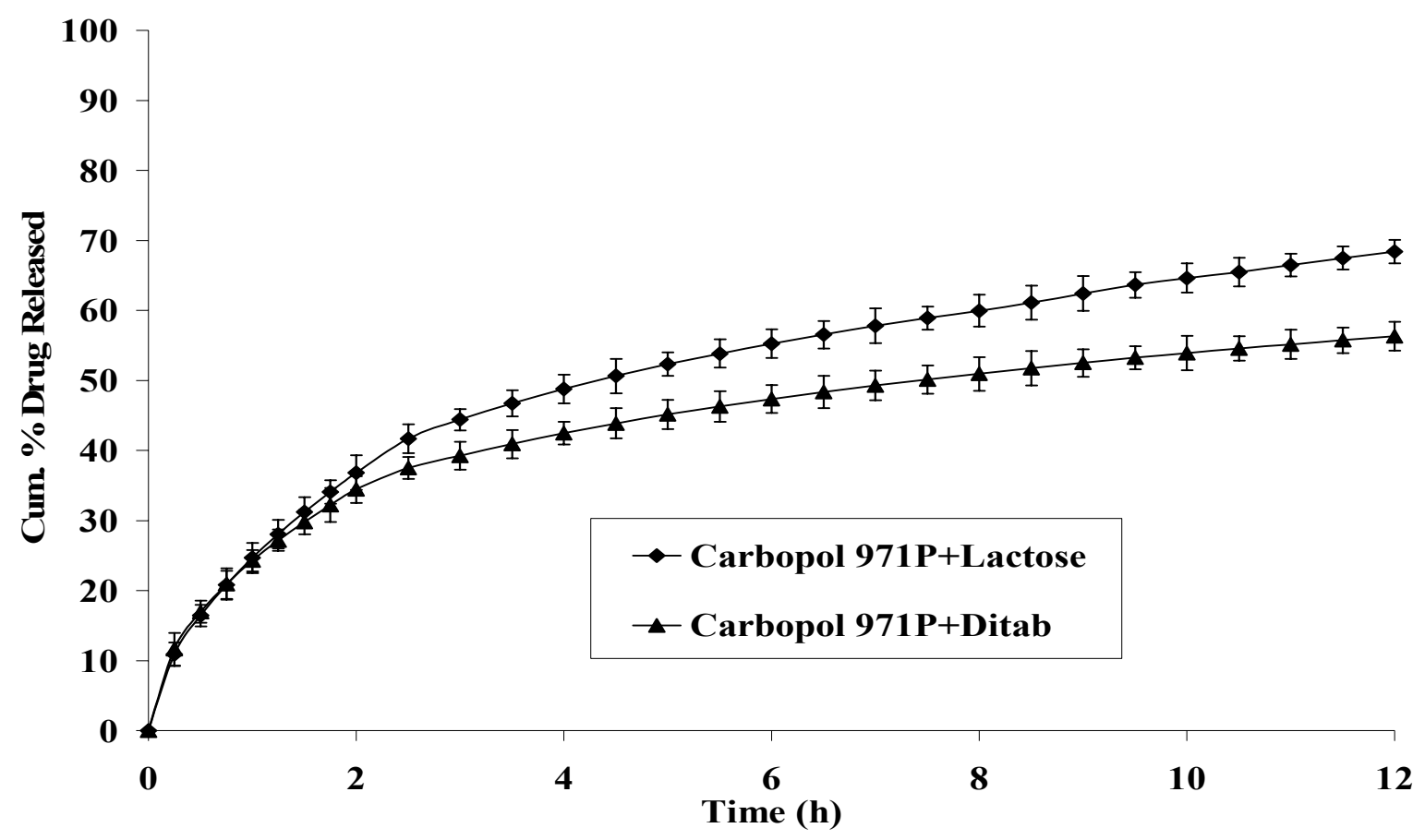

Figure 3.8. Effect of fillers on formulations containing carbopol 971P $\uparrow$

$\dagger$ Each data point represents average of three measurements. Standard deviation of three measurements is presented as error bars. 


\subsubsection{Formulations containing carbopol $974 P$ as release controlling polymer}

In the second step, the type of carbopol, release controlling polymer, was changed. In all aforementioned formulations, carbopol $971 \mathrm{P}$ was used as release controlling polymer. Carbopol $971 \mathrm{P}$ is less crosslinked compared to carbopol 974P. Hydrogels formed from carbopol 971P has "fishnet" structure while the hydrogels formed from carbopol 974P has "fuzzball" structure. Polymer chains of hydrogels with "fishnet" structure have more mobility compared to the hydrogels of "fuzz ball" structure. Free mobility of polymer chains increases the probability of complexation between ionized polymer and the drug molecule. Thus, polymers with less crosslinks will impede the drug release to higher extent compared to polymers with high crosslinks. In formulation containing lactose monohydrate as filler and carbopol 971P as release controlling polymer the change was made. Carbopol 971P was replaced by carbopol 974P, a highly crosslinked grade of carbopol. Comparison of release profiles of formulations containing carbopol 971P and carbopol 974P is given in Figure 3.9. The Figure 3.9 clearly illustrates the complete drug release was achieved at the end of the dissolution. Comparison between the USP standards and dissolution profile of formulation containing lactose monohydrate as filler and carbopol $974 \mathrm{P}$ as release controlling polymer is given in Table 3.8.

Although the USP compliant sustained release (SR) formulation was developed by combining highly cross linked grade of carbopol and soluble filler, it is not clear the final result is due to change in the carbopol grade or due to the change in the filler solubility.

Thus, one more formulation was prepared using carbopol 974P as release controlling polymer however using dicalcium phosphate, water insoluble filler, as filler in the formulation. It was hypothesized that the complete drug release is due to the replacement of carbopol 971P by carbopol 974P then the change in filler must still a formulation that can release the drug 


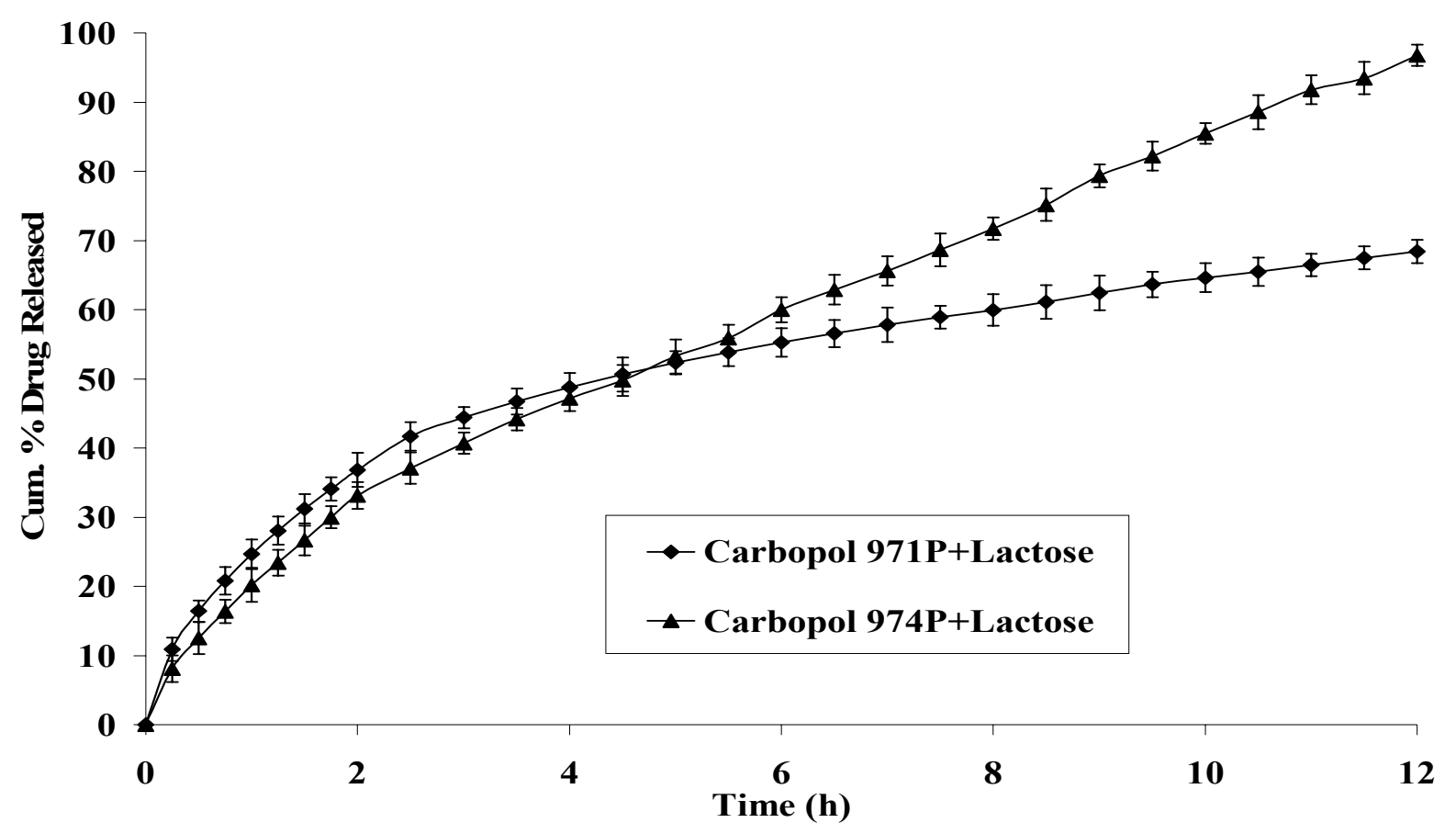

Figure 3.9. Effect of carbopol grade on dissolution profiles $\uparrow$

$\dagger$ Each data point represents average of three measurements. Standard deviation of three measurements is presented as error bars. 
Table 3.8. Comparison between optimized formulation and USP specifications

\begin{tabular}{ccc}
\hline Time & $\begin{array}{c}\text { USP requirement } \\
(80 \text { mg SR tablet })\end{array}$ & $\begin{array}{c}\text { Formulation with 10\% 974P } \\
\text { with lactose }\end{array}$ \\
\hline $1 \mathrm{~h}$ & Less than $20 \%$ & $20.21 \pm 2.36 \%$ \\
$6 \mathrm{~h}$ & $20-45 \%$ & $40.71 \pm 2.84 \%$ \\
$6 \mathrm{~h}$ & $45-60 \%$ & $58.28 \pm 2.75 \%$ \\
$12 \mathrm{~h}$ & Not less than $80 \%$ & $96.79 \pm 3.67 \%$ \\
\hline
\end{tabular}


completely at the end of $12 \mathrm{~h}$. Otherwise, the complete drug release is attributed to both solubility of filler and reduction in the extent of complexation between drug and carbopol. The formulation containing dicalcium phosphate as tablet filler did not yield complete drug release at the end of $12 \mathrm{~h}(58.96 \%$ drug release at $12 \mathrm{~h})$. Therefore, it is confirmed that complete drug release is an effect of water solubility of the filler and reduction in the complexation of drug with carbopol.

\subsection{CONCLUSION}

Sustained release matrix tablets of propranolol hydrochloride can be developed using carbopol - wax blends. Mechanistic studies confirmed insignificant relationship between water absorption profiles and drug release profiles from formulations containing carbopol $971 \mathrm{P}$ as release controlling polymer. Little or no matrix erosion contributed to incomplete drug release from tablets containing carbopol 971P. Absence of matrix erosion and high degree of complexation resulted in incomplete drug release in formulations containing carbopol 971P as release controlling polymer. Sustained release formulation showing USP compliant drug release profiles was prepared using appropriate carbopol grade (carbopol 974 P) and filler (lactose monohydrate). Thus, selection of appropriate carbopol grade and soluble filler were pivotal in developing USP compliant formulation of propranolol hydrochloride. 


\section{Chapter 4: Formulation Development of Glipizide}

\subsection{INTRODUCTION}

Type 2 diabetes is one of the most prevalent diseases in the modern world. Diabetes is currently the fourth leading cause of death by disease in the United States. Type 2 diabetes represents $98 \%$ of all diabetes among patients older than 45 years of age.

There are two widely accepted hypotheses about origin of type 2 diabetes. First one is related to insufficient insulin secretion from beta cells. The second hypothesis is related to insulin resistance in the peripheral tissues. The beta cells initially compensate the normal glucose metabolism by increasing insulin secretion. However, when the demand for insulin exceeds the ability of beta cells to compensate, it leads to pancreatic exhaustion.

As first line of defense for diabetes, diet and exercise regimen is recommended to patients. Upon six weeks course of diet and exercise plan, patients will show improvement if there is any. If the patients fail to achieve near target blood glucose levels from diet and exercise regiment, then pharmacotherapy is added to diet and exercise plan. Pharmacotherapy includes oral anti diabetic drugs. Physicians must consider benefits of each drug against cost, contraindications, degree of glycemic-lowering needed, ease of compliance, duration of action, patient's weight and patient's lipid profiles.

The sulphonylureas and biguanides are the most commonly prescribed categories of medications for oral anti diabetic therapy. They are safe, have better tolerability and superior pharmacodynamic effects. Sulfonyl ureas stimulate the release of insulin from the pancreatic islet cells via stimulation of receptors. Glipizide is one of the commonly prescribed anti diabetic drugs from short acting sulphonyl urea category. Short acting sulphonyl urea drugs have some 
advantages compared to long acting sulphonyl urea drugs. Short acting sulphonyl ureas are more efficient in increasing meal-stimulated insulin secretion and have lower risk of hyperglycemia.

Glipizide was synthesized chemically in 1971 and has excellent safety profile. Glipizide is an effective therapeutic agent for both first phase insulin secretion and for providing sustained stimulatory response during long term treatment. Blood glucose concentration starts to decrease within 30 min of ingestion of glipizide. Glipizide is 100 times more potent than tolbutamide. Glipizide has uniform, rapid absorption throughout the gastro intestinal tract. Peak plasma concentration of Glipizide is observed within 1-3h after single oral dose. Half life of Glipizide is 2 to $4 \mathrm{~h}$ in both intravenous and oral administration. Glipizide has no plasma accumulation following repeated oral dosing. In spite of its clinical efficiency, Glipizide has poor patient compliance because of its multiple daily dosing. Thus, it is appropriate to formulate Glipizide in controlled release formulation. Glipizide is commercially available as controlled release formulation.

Glipizide is a weakly acidic, water insoluble drug. It belongs to class II drugs of biological classification system (BCS). Class II drugs have poor solubility and high permeability. Commercial controlled release formulation of Glipizide uses osmotic drug delivery technology. Commercial controlled release formulation of Glipizide is a coated bilayer tablet (Push-pull system). One layer contains drug and the other layer contains osmogen and other ingredients required to push the drug through the laser drilled hole. The tablet is coated with cellulose acetate. Cellulose acetate is semi-permeable i.e., it is permeable only to water not for other substances. Upon water entry into the tablet through semi-permeable membrane, the osmogens dissolve and create osmotic pressure inside the tablet. Osmotic pressure drives the drug out of the tablet through the laser drilled delivery orifice. Osmotic drug delivery systems are difficult to 
manufacture and they need special equipments for manufacturing. The objective of this work is to develop bioequivalent formulation of Glipizide to its commercial formulation, Glucotrol ${ }^{\circledR}$ XL. And, it is also of our interest to develop controlled release matrix formulation using carbopol as release controlling polymer and hot melt granulation technology.

As mentioned in formulation development of propranolol hydrochloride, hot melt granulation technique is a versatile technique offering many formulation options. However, the excipients must be selected based on the properties of drug and the requirements of controlled release. Following section presents rationale for selection of binder and fillers in the formulation

\subsection{SELECTION OF FORMULATION COMPONENTS}

\subsubsection{Selection of binder}

Glipizide is a poorly soluble weakly acidic drug. Glipizide has $\mathrm{pH}$ dependent solubility. Solubility of the glipizide is $0.078 \mathrm{mg} / \mathrm{ml}$ in $\mathrm{pH} 6.8$ and it is virtually insoluble in $\mathrm{pH} 1.2$. In hot melt granulation technique, waxy material is used as binder. Waxy binder can be either hydrophilic e.g. Gelucire with higher HLB value or hydrophobic e.g. waxes with lower HLB values. In this case, the objective is to formulation controlled release formulation of Glipizide that is bioequivalent to osmotic delivery system of Glipizide. Osmotic delivery system has constant release rate for $14 \mathrm{~h}$. Therefore, the ingredients used in the formulation, must not interfere with the dissolution of the drug as it may result in formulation that will have very low release rate. If a hydrophobic wax is selected as binder in the formulation, then a dispersion of drug in the hydrophobic wax will be formed. This will hamper the water availability for the 
dissolution of drug particles. Therefore, hydrophobic waxes must be avoided in the formulation. Gelucire 50/13, a hydrophilic wax, is selected as binder for the formulation.

\subsubsection{Selection of filler}

Glipizide is a low dose drug. It is administered in three doses of $2.5 \mathrm{mg}, 5 \mathrm{mg}$ and $10 \mathrm{mg}$. In this study, our objective is to develop bioequivalent formulation for $10 \mathrm{mg}$ dose. However, it is difficult formulate such a low dose without a filler. Fillers are available in various choices. They are: (i) Water insoluble, non-swellable e.g. Dicalcium phosphate, (ii) Water insoluble, water swellable e.g. Microcrystalline cellulose, (iii) Water soluble, non-swellable e.g. Lactose monohydrate. As mentioned earlier, inclusion of water insoluble material will hamper the availability of water to the drug dissolution. In order to achieve complete drug release from a formulation containing poorly soluble drug, the excipients in the formulation should not interfere with the drug dissolution. Therefore, dicalcium phosphate is not a good choice for filler and either microcrystalline cellulose or lactose monohydrate can be selected as fillers.

Microcrystalline cellulose is depolymerized cellulose and has tremendous water uptake capacity. Since microcrystalline cellulose promotes water influx into the system, the release controlling mechanism in matrix tablet will be ruined. Matrix systems control the drug release by modulating the water influx and controlling the drug efflux. Therefore, in order to compensate for water influx, higher polymer concentration will be needed. This will increase the cost of the delivery system. Hence, unless justified, microcrystalline cellulose is not a filler of choice. On contrary, lactose monohydrate is water soluble filler and it dissolves slowly in water. Lactose absorbs less water compared to microcrystalline cellulose however it does not impede the water influx into the system like dicalcium phosphate. As lactose dissolves, it creates pores in the 
delivery system and these pores aid drug release. Since lactose dissolves slowly, the extent of pore creation does not ruin the controlled release properties of release controlling polymer. Therefore, lactose monohydrate is filler of choice for the formulation.

\subsection{MATERIALS AND METHODS}

\subsubsection{Materials}

Glipizide, Carbopol 971P (Noveon, Cleveland, OH), Gelucire 50/13 (Gattefosse, Paramus, NJ), Lactose monohydrate (Foremost, Baraboo, WI), Microcrstalline cellulose (MCC) (Avicel® PH-101) (FMC corporation, Philadelphia, PA).

\subsubsection{Methods}

\subsubsection{Preparation of tablets}

Hot melt granulation technique was used to prepare granules from the blend containing appropriate quantities of Glipizide, Carbopol 971P, filler and Gelucire 50/13. Ingredients and composition of formulations are given in the Table 4.1 and Table 4.2 respectively. Ingredients of each formulation except the waxy binder were mixed for two minutes in the reverse mode at 1500 rpm prior in a Robot-Coupe ${ }^{\circledR}$ high shear mixer-granulator (Robot-Coupe Inc., Jackson, MS). After blending, a circulating water bath was attached to the granulator bowl. Temperature of the bowl was monitored using a digital thermometer. Once the temperature of the bowl reached $60^{\circ} \mathrm{C}$, waxy binder was added to the powder blend. Bowl temperature was kept at $60^{\circ} \mathrm{C}$ during granulation. Granulation was carried out in the reverse mode at $1500 \mathrm{rpm}$ for $2 \mathrm{~min}$. 
Table 4.1. Ingredients of formulations for glipizide product development

\begin{tabular}{|c|c|c|c|c|}
\hline Batch code & Filler & Tablet weight & Drug - polymer ratio & $\begin{array}{l}\text { Polymer- } \\
\text { wax ratio }\end{array}$ \\
\hline L-1 & Lactose & 100 & $1:: 0.5$ & $1:: 1$ \\
\hline L-2 & Lactose & 100 & $1:: 1$ & $1:: 1$ \\
\hline L-3 & Lactose & 400 & $1:: 0.5$ & $1:: 1$ \\
\hline $\mathrm{L}-4$ & Lactose & 400 & $1:: 1$ & $1:: 1$ \\
\hline M-1 & Avicel & 400 & $1:: 1$ & $1:: 1$ \\
\hline M-2 & Avicel & 400 & $1:: 2$ & $1:: 1$ \\
\hline M-3 & Avicel & 400 & $1:: 4$ & $1:: 1$ \\
\hline M-4 & Avicel & 400 & $1:: 6$ & $1:: 1$ \\
\hline
\end{tabular}


Table 4.2. Compositions of formulations for glipizide product development

\begin{tabular}{ccccc}
\hline Batch code & Drug $(\%)$ & Carbopol $(\%)$ & Gelucire (\%) & Filler (\%) \\
\hline L-1 & 10.00 & 5.00 & 5.00 & 80.00 \\
L-2 & 10.00 & 10.00 & 10.00 & 70.00 \\
L-4 & 2.50 & 1.25 & 1.25 & 95.00 \\
M-1 & 2.50 & 2.50 & 2.50 & 92.50 \\
M-2 & 2.50 & 2.50 & 2.50 & 92.50 \\
M-3 & 2.50 & 5.00 & 5.00 & 87.50 \\
M-4 & 2.50 & 10.00 & 10.00 & 77.50 \\
\hline
\end{tabular}


The resulting granules were passed through a 20-mesh sieve to remove any coarse granules while they were still warm. The sieved granules were allowed to cool to room temperature. Tablets from aforementioned granules were prepared using an 18-station rotary tablet press equipped with 5/16" deep-concave punches and dies was used for tablet compression.

\subsubsection{Evaluation of in vitro dissolution}

Tablets were tested for its dissolution characteristics using USP apparatus II (paddle). Hydrochloric acid $(0.1 \mathrm{~N}, 750 \mathrm{ml})$ was used as the dissolution media for the first 2 hours. Then, the $\mathrm{pH}$ of the dissolution media was adjusted to 6.8 using $250 \mathrm{ml}$ of $1 \mathrm{~N}$ tribasic phosphate solution. Drug dissolution from the tablets was determined at $37^{\circ} \mathrm{C}$ at $50 \mathrm{rpm}$. Drug release from the tablets was monitored using in situ fiber optic UV probes equipped with PDA (Photo diode array) detectors (PION, Inc., Woburn, MA). Estimation of the drug concentration in the dissolution media was based on single point calibration at $288 \mathrm{~nm}$.

\subsection{RESULTS AND DISCUSSION}

\subsubsection{Lactose based formulations}

Composition of formulations with lactose as filler is given in the Table 4.2. Formulations $\mathrm{L}-1$ and $\mathrm{L}-2$ have $100 \mathrm{mg}$ tablet weight. Both formulations contain different drug to polymer ration. While $\mathrm{L}-1$ contains 1:0.5 drug-polymer ratio, $\mathrm{L}-2$ contains $1: 1$ drug to polymer ratio. In vitro dissolution profiles of the formulations are presented in Figure 4.1. These $100 \mathrm{mg}$ sustained release tablets containing lactose as filler had long lag time. Only less than $20 \%$ 


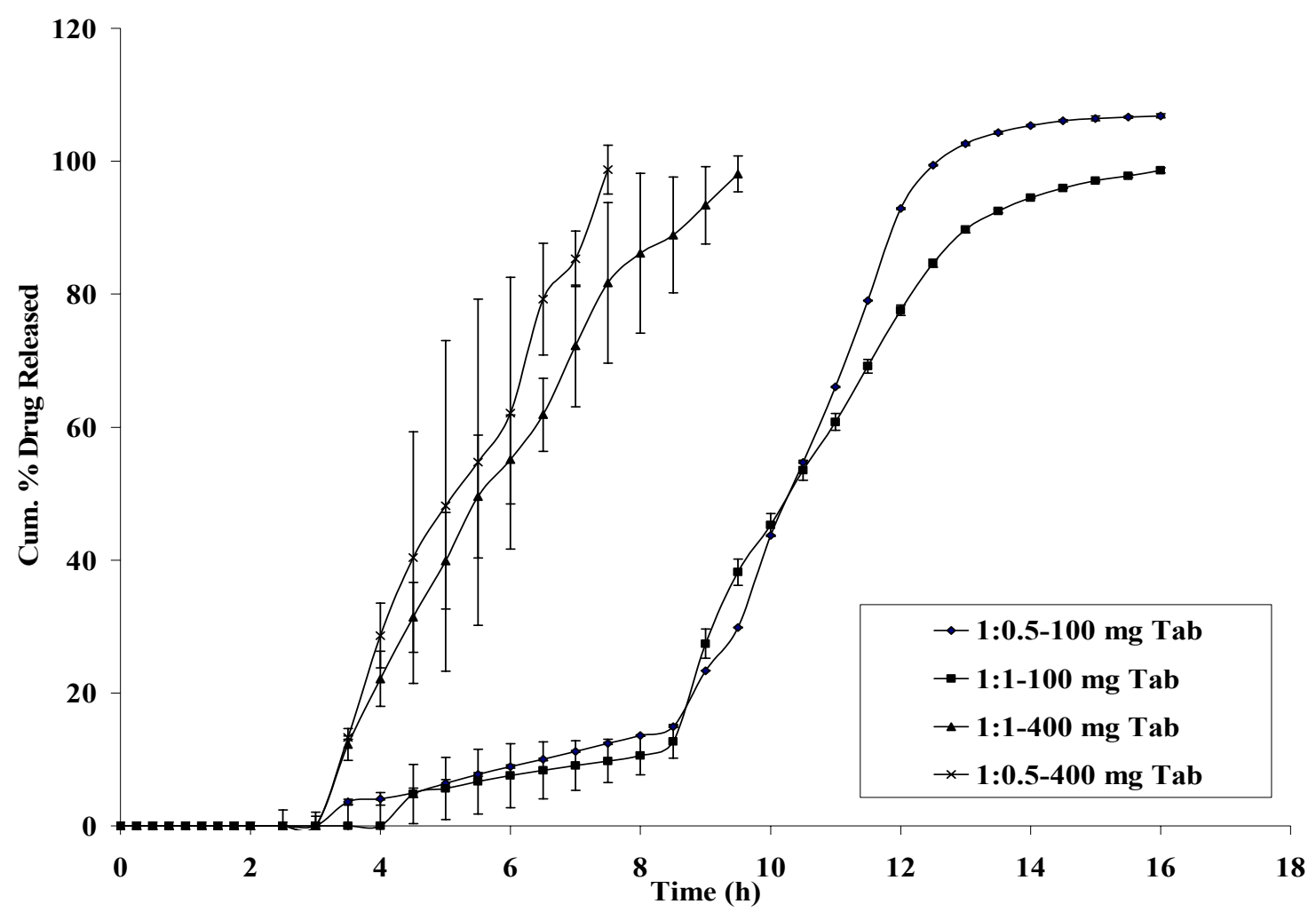

Figure 4.1. Dissolution profiles of formulations containing lactose as filler $\dagger$

$\dagger$ Each data point represents average of three measurements. Standard deviation of three measurements is presented as error bars. 
drug was released from the tablets till $8 \mathrm{~h}$. Then, the matrix started to disintegrate and dump the drug out. Within $12-14 \mathrm{~h}$, the drug release from formulations was almost complete. This can be attributed to higher solubility of lactose monohydrate compared to drug solubility. In phosphate buffer, Lactose solubility is $148 \mu \mathrm{g} / \mathrm{ml}$ and the Glipizide solubility is $78 \mu \mathrm{g} / \mathrm{ml}$. When dissolution media enters into the matrix, there is a competition between the lactose and glipizide. Since lactose has more solubility than glipizide, it dissolves faster than glipizide. This reduces the availability of dissolution media for the dissolution of glipizide hence dissolution of glipizide. As lactose continues to dissolve and leave the matrix, it creates pores that weaken the matrix structure. In addition, dissolution of lactose increases the osmotic gradient and with draws more dissolution media into the system. The continuous increase in osmotic pressure weakens the matrix and results in matrix disintegration and dose dumping after certain time $(8 \mathrm{~h})$. Thus, the $100 \mathrm{mg}$ formulations containing lactose as filler has two problems

i. Insufficient availability of the dissolution media for drug dissolution in the initial period

ii. Competitive reduction in drug dissolution.

Although competitive reduction in drug dissolution can not be rectified due to the lactose's higher solubility, dissolution media influx into the matrix can be increased. Increase is dissolution media influx will provide more dissolution media for dissolution of drug hence improve the drug release. Dissolution media influx can be increased by increasing the lactose content in the formulation. Therefore, the tablet weight was increased to $400 \mathrm{mg}$ by increasing the lactose content in the formulations. However, for comparative purposes, the drug-polymer ratio is kept at constant level of 1: 0.5 and 1:1 drug-polymer ratio.

Formulations $\mathrm{L}-3$ and $\mathrm{L}-4$ were $400 \mathrm{mg}$ tablets containing drug - polymer ratio of 1:0.5 and 1:1 respectively. In vitro dissolution profiles of formulations $\mathrm{L}-3$ and $\mathrm{L}-4$ are given 
in Figure 4.1. As expected, $400 \mathrm{mg}$ formulations containing more lactose released drug faster than $100 \mathrm{mg}$ formulations. No lag period was observed in drug release in dissolution media $\mathrm{PH}$ 6.8. However, the drug release was complete in less than $10 \mathrm{~h}$. To be bioequivalent to osmotic system of glipizide, the matrix formulation must have controlled drug release for $14 \mathrm{~h}$. Faster drug release from $400 \mathrm{mg}$ lactose based formulations can be attributed to insufficient polymer content to control the drug release. Therefore, by increasing polymer content or decreasing lactose content, controlled release formulation of glipizide can be developed. And the filler content and polymer content can be optimized to yield bioequivalent formulation of glipizide. However, the controlled release from the formulation will be dependent on the controlling drug solubility based on dissolution media influx in to the matrix tablets. In in vivo conditions, composition, volume and $\mathrm{pH}$ of the gastric contents may change in fast and fed conditions. In such circumstances, the dissolution profile glipizide from lactose based formulation will also change. Therefore, the lactose based formulations are not robust. In addition, all formulations using lactose as filler had picking problems and low tablet hardness ( 3 to $4 \mathrm{kps}$ for $400 \mathrm{mg}$ tablets). Although it can be solved by addition of lubricants and glidants, no such attempts were made as the release profiles from lactose based formulation lack scope for further development.

\subsubsection{Microcrystalline cellulose based formulations}

Filler of $400 \mathrm{mg}$ formulations was changed to microcrystalline cellulose from lactose monohydrate. Change of filler to microcrystalline cellulose yielded tablets with good hardness (9 to $11 \mathrm{kps}$ ) and no picking or sticking problem was observed during compression. However, as expected, the drug release from microcrystalline cellulose based formulations was faster. This can be attributed to increase in dissolution media influx into the system caused by the presence 
of microcrystalline cellulose. To control the influx of the dissolution media and efflux of the drug, polymer content was increased and four formulations were prepared. The composition of formulations is given in Table 4.2 and their release profiles are given in Figure 4.2.

Formulations M 1 to M 4 have differences in drug-polymer ratio. They had drug-polymer ratio of $1: 1,1: 2,1: 3$ and 1:6. Increase in drug - polymer ratio reduced the release rate. Formulation M4 containing 1:6 drug - polymer ratio had closest release profile to commercial formulation. Bioequivalence of the microcrystalline cellulose based formulations were checked using F-2 values. F-2 values were calculated using following formula and drug release from commercial formulation as reference.

$$
f_{2}=50 \log \left\{\left[1+\frac{1}{n} \sum_{t=1}^{n} w_{t}\left(R_{t}-T_{t}\right)^{2}\right]^{-0.5} \times 100\right\}
$$

Where $\mathrm{n}$ is the number of sampling time points used. In this study, $\mathrm{n}$ is equal to $10 . \mathrm{R}_{\mathrm{t}}$ is the actual cumulative percentage of acetaminophen released from the beads at each of the selected $n$ time points. $T_{t}$ is the ANN model predicted cumulative percentage of acetaminophen released from the beads at each of the selected $\mathrm{n}$ time points. $\mathrm{w}_{\mathrm{t}}$ is the optional weight factor. In this study, wt is equal to 1 .

When the two profiles are identical, $f_{2}=100$. The $f 2$ value is equal to 50 when an average difference of $10 \%$ between the dissolution profiles that are being compared, is observed at all time points used for the calculation of the $f_{2}$ value. The FDA has set a public standard of $f 2$ value between 50 and 100 to indicate similarity between two dissolution profiles. A formulation is considered bioequivalent if the F2 values are above 50 . 


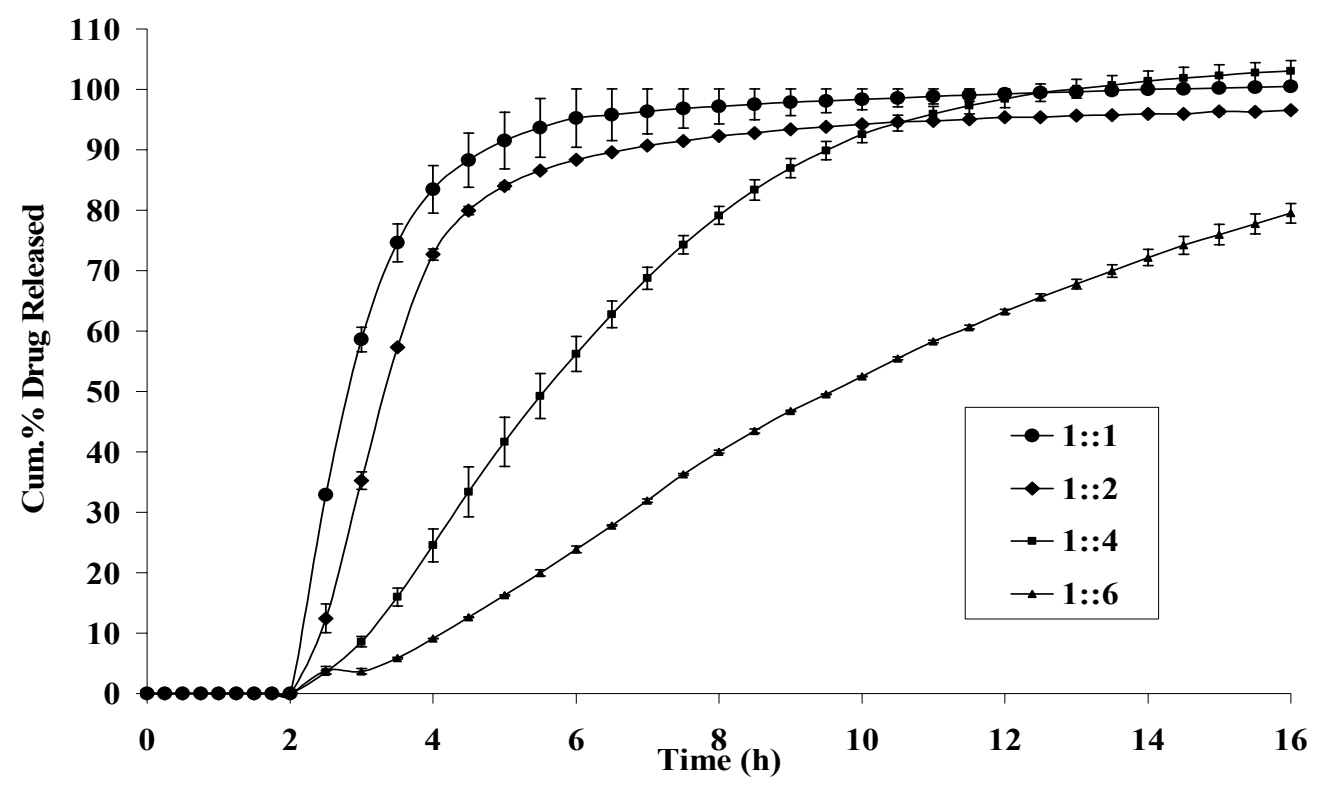

Figure 4.2. Dissolution profiles of formulations containing MCC as filler $\dagger$

$\uparrow$ Each data point represents average of three measurements. Standard deviation of three measurements is presented as error bars. 
Based on this criterion, formulation $M-4$ containing drug - polymer ratio of 1:6 is bioequivalent to commercial formulation. Although proven bioequivalent, formulation $\mathrm{M}-4$ had incomplete drug release. Average drug release of $79.49 \%$ was observed at the end of $16 \mathrm{~h}$. Therefore, to maximize the drug release with out significantly changing F-2 value, artificial neural network model was developed and optimization of the formulation composition was done using genetic algorithm and artificial neural network model. Drug - polymer ratio of microcrystalline cellulose formulations and their F2 values are given in the Table 4.3.

\subsubsection{Formulation optimization}

\subsubsection{Artificial neural network modeling (ANN)}

Artificial neural network modeling is an artificial intelligence based modeling tool. It uses similar techniques to that of normal human learning behavior. That is, initially the trend in the data is identified using a set of data called "training set" then the model is validated for further use. It is commonly used by pharmaceutical scientists for solving various problems. Drug release optimization is one of the challenging problems that will require ANN modeling.

Artificial neural network modeling is a versatile modeling tool. Both linear and nonlinear functions can be modeled using ANN. ANN models contain three components; input layer, hidden layer and output layer. Input layer contains independent factors of the model and the output layer contains dependent variables of the model. Hidden layer comprises of mathematical transformations called neurons. Each neuron represents a mathematical transformation. Neurons connect input layer and output layer. Independent variables are mathematically transformed as they are connected with output layer through neurons. 
Table 4.3. F2 values of formulations M 1 to M 4

\begin{tabular}{ccc}
\hline Batch code & Drug - polymer ratio & F - 2 value \\
\hline$M-1$ & $1: 1$ & 19.59 \\
$M-2$ & $1: 2$ & 22.84 \\
$M-3$ & $1: 4$ & 33.66 \\
$M-4$ & $1: 6$ & 52.16 \\
\hline
\end{tabular}


After the mathematical transformation, the relationship between input factors and output loses logical meaning. Therefore, the ANN models have no statistical meaning hence sometimes they are called "black box". However, the predictability of the ANN models is superior to conventional statistical models. The exceptional predictability of ANN models have been attributed to selection of appropriate mathematical transformations and input scaling functions of ANN modeling. When a set of input factors and output are presented to ANN model, the input factors are scaled down to reduce the dimension of input space. These scaling functions are called "squashing" functions and they convert the numerical values of inputs to symbols representing low and high ranges of the input. For example, if the one of the inputs of ANN model is $25,40.60$ and 75 , then the range is fist computed and in this case it is 25 to 75 . Low value of 25 is assigned a value of -1 and the high value of 75 is assigned +1 and the values in between take corresponding values in this scale. Squashing of inputs normalizes the inputs and reduces the dimension. Following input scaling, the scaled inputs are transformed in hidden layers. Transformation of scaled input is done by mathematical functions. There are many mathematical functions that are used for transforming inputs and sigmoid transformation, tansigmoid transformation, log transformation are few popular transformation functions. Selection of mathematical function is dependent on the error distribution. That is, an appropriate input transformation is selected to minimize the error in the training set. The data presented to ANN model is generally segmented using random sampling in to three sets; training set, test set and validation set. Initially, the transformation functions are selected based on the input-output relationship in the test set and a random weight is added to the transformation functions. Then, output values are predicted using an equation containing these transformations and randomly generated weights. An error distribution is calculated from predicted values and actual output 
values in the test set. Then this error function is minimized by adjusting weights and/or changing transformations. The modifications in weight and transformation functions are done till maximum minimization of error function is achieved. This process of adjusting weights and choosing transformation is called "training". The training will stop once the error function reaches minimum value. After the training stops, the ANN model can be validated using an external validation set.

ANN model training is similar to human brain's learning. That is, the relationship pattern between inputs and outputs is studied from known examples (training set). However, ANN model use mathematical functions with no logical justification unlike human brain where relationship patterns are associated with logic. This difference in learning between human brain and ANN model has advantage and disadvantage. Advantage is the numerical accuracy in predictions which is higher than the human brain. Disadvantage is "overlearning". Overlearning is a process of extended training leading to selection of wrong input-output relationship pattern. Overlearning happens when error in the data distribution "mislead" the ANN model to select wrong transformation functions. This will result in learning from error rather than true inputoutput relationship. The outcome of "overlearning" is lack of generalization which is manifested as least training error and high error in predictions with validation set. In most of the softwares used for ANN model building, training schemes are tweaked to periodically consult with independent validation set to stop the training process in early stages of training before overlearning begins. One of the easiest tweaks in the training scheme to prevent overlearning is "leave-one-out" cross validation. In "leave-one-out" cross validation, test set is divided into test set and an internal validation set. While test set is used to adjust training weights, internal validation set is used to terminate the training process before the overlearning begins. This 
process is continued by using validation set as part of training set and using another part of data as validation set. This is one of the effective means for preventing over learning.

\subsubsection{Formulation optimization using $A N N$}

Objective of current ANN model is to identify the formulation that can release drug completely. AI Triology® software program (Ward systems, Frederick, MD) was used for ANN model building. AI Triology ${ }^{\circledR}$ uses backpropagation training and generalized regression training algorithms. Earlier studies on selection of training algorithm for training indicate that generalized regression algorithm had better generalization than backpropagation algorithm. Therefore, only generalized regression algorithm was used for model development in this study. Following model development and leave one out cross validation, ANN model showing least prediction error was selected for formulation optimization. Genetic algorithm was used for formulation optimization. Drug release profile of commercial formulation was used as reference. Constraints were added on the later time points to support the optimization algorithm to find regions where results that can fulfill the objective of the current study i.e., to yield a formulation that can release the drug completely. Optimization based on ANN model yielded the formulation composition containing $11.25 \%$ carbopol with $2.5 \%$ drug loading and $400 \mathrm{mg}$ tablets containing microcrystalline cellulose as filler. Figure $\mathbf{4 . 3}$ presents the comparison of predicted dissolution profiles of ANN predicted formulation and the actual dissolution profiles of the ANN predicted formulation. $\mathrm{F}-2$ values between these predicted and actual formulation was $74.34 \%$. This confirms the validity of the ANN model. 


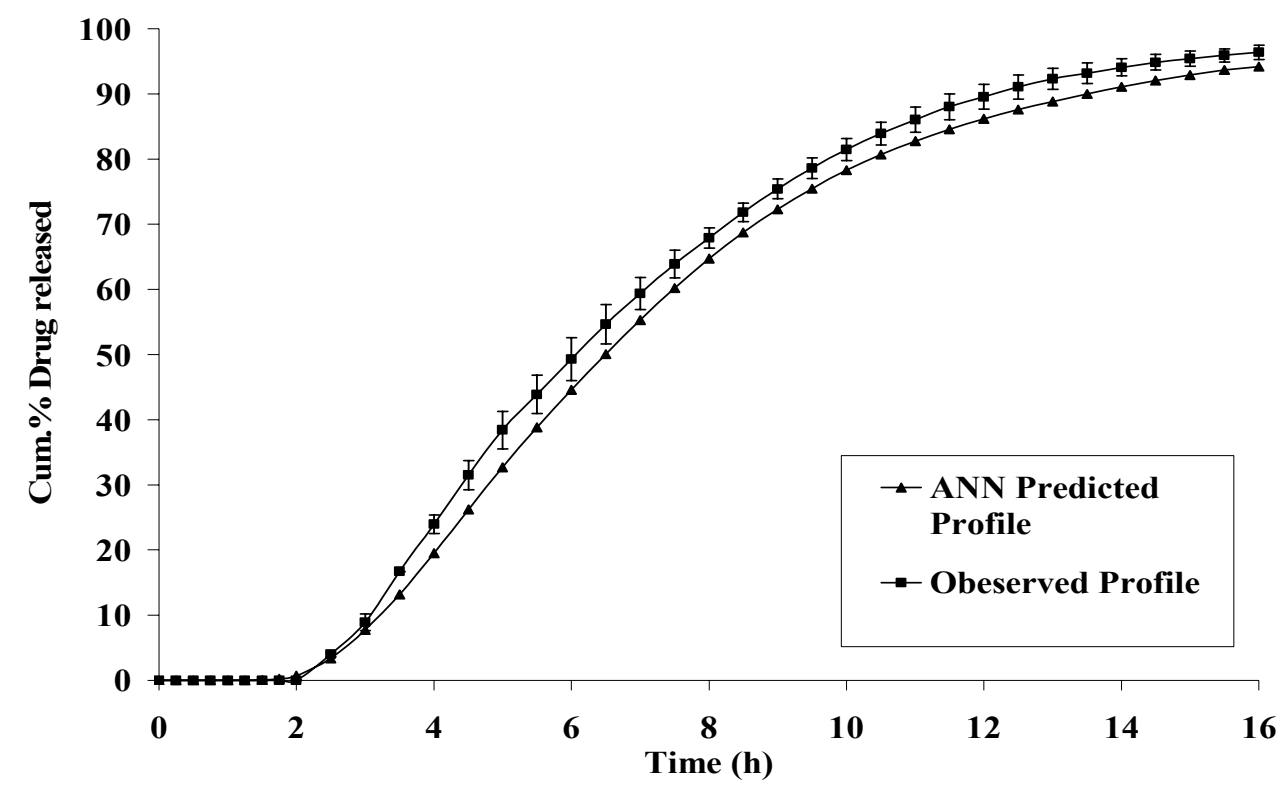

Figure 4.3. Comparison of ANN predicted and actual dissolution profiles $\uparrow$

$\dagger$ Each data point represents average of three measurements. Standard deviation of three measurements is presented as error bars. 


\subsubsection{Effect of compression force on the drug release}

Literature reports that the drug release from carbopol matrix is sensitive to the compression force. This is attributed to change in the interstitial space in higher compression force. Higher the compression forces lower the interstitial space between polymer particles in the tablet. This will boost the controlled release performance of the polymer. Thus it is of our interest to test the effect of compression force on the drug release of ANN optimized formulation. Therefore, $400 \mathrm{mg}$ tablets containing $11.25 \%$ of carbopol and microcrystalline cellulose as filler were compressed at two more additional compression forces of $1 \mathrm{mT}$ and 1.5 $\mathrm{mT}$. Although only marginal increase in tablet hardness was achieved, significant changes in the in vitro dissolution profile was observed when the compression force was increased to $1 \mathrm{mT}$ from $0.5 \mathrm{mT}$. However, there is less changes in the drug release was observed when the compression force was increased to $1.5 \mathrm{mT}$ from $1 \mathrm{mT}$. Figure 4.4 presents the effect of compression force on drug release profiles of the ANN predicted formulation. Table 4.4 presents F-2 values of these formulations against commercial formulation. Formulation containing $11.25 \%$ of carbopol and microcrystalline cellulose as filler and prepared with both $1 \mathrm{mT}$ and 1.5 $\mathrm{mT}$ were bioequivalent to commercial formulation.

\subsection{CONCLUSIONS}

Bioequivalent formulations of glipizide were prepared using carbopol - wax blends. Lactose based formulations either released drug quickly or had long lag time. Lactose based formulations had poor tablet hardness and picking problem during compression. Microcrystalline cellulose based formulations yielded tablets with better hardness compared to lactose based formulations and had no picking problem during compression. Drug release from 


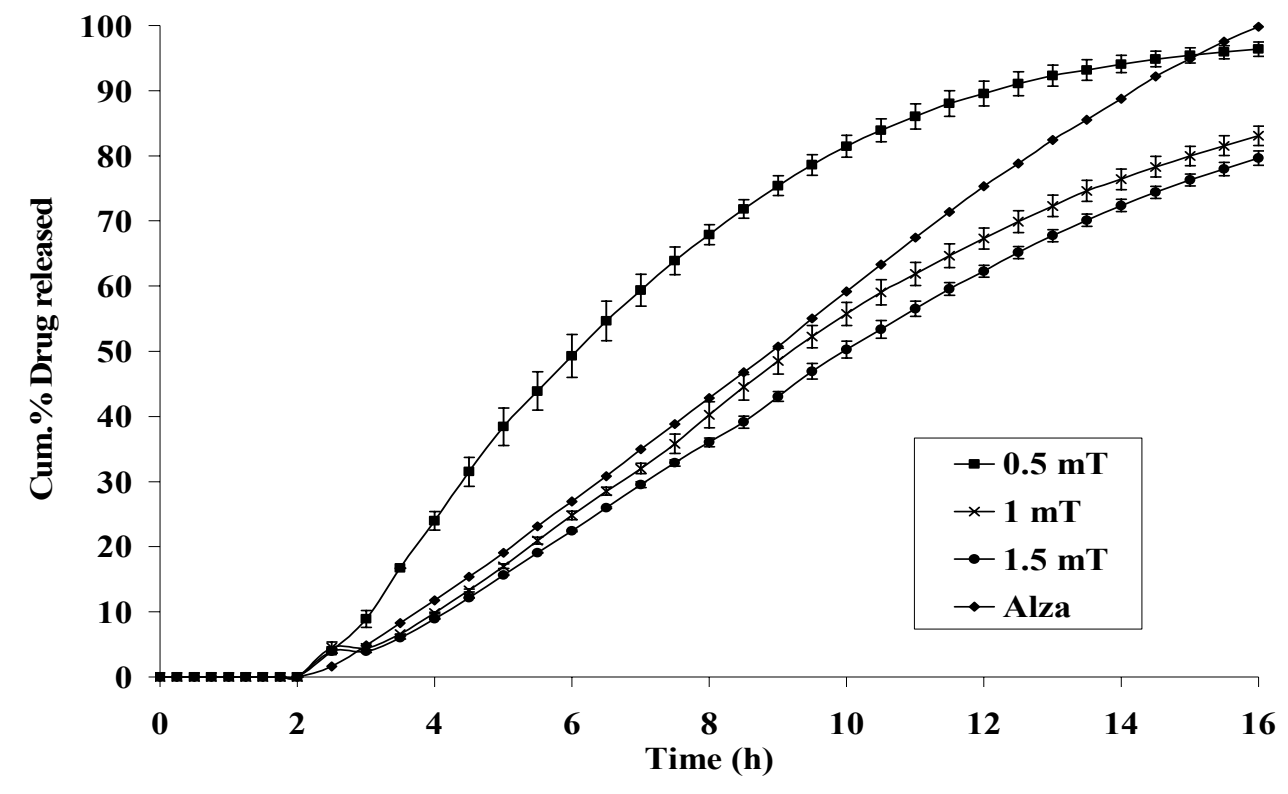

Figure 4.4. Effect of compression force on dissolution profiles $\uparrow$

$\dagger$ Each data point represents average of three measurements. Standard deviation of three measurements is presented as error bars. 
Table 4.4. F2 values of batches prepared using different compression forces

\begin{tabular}{ccc}
\hline Compression force & F -2 value & Drug release at $16 \mathrm{~h}$ \\
\hline $0.5 \mathrm{mT}$ & 41.42 & $96.41 \%$ \\
$1 \mathrm{mT}$ & 58.47 & $83.07 \%$ \\
$1.5 \mathrm{mT}$ & 50.89 & $79.65 \%$ \\
\hline
\end{tabular}


microcrystalline cellulose based formulations was proportional to the drug - polymer ratio. Drug release from microcrystalline cellulose based formulations was related to compression force used to compress the tablets. Increasing the compression force reduced the extent of drug release and yielded zero order release formulations. 


\section{Chapter 5: Non Destructive Prediction of Dissolution Profiles Using NIRS}

\subsection{INTRODUCTION}

Near Infrared Spectroscopy (NIR) has been used in the pharmaceutical industry for both quality control and process monitoring at in-line, on-line and at-line locations to control the quality attributes of products and by-products. It is an attractive quality control tool because of its versatility, quickness and absence of sample preparation.

Literature suggests NIR can be used in various stages of tablet dosage form manufacture. NIR can be used in raw material identification, determination of water content in raw materials, identification of impurities or isomeric forms of raw materials, monitoring blend uniformity in mixing operation, estimating granule size and density changes in granulation process, measuring the rate of drying in the drying operation, estimating hardness, drug content, disintegration and dissolution of tablets, monitoring coating thickness in coating operation etc.

In spite of the availability of substantial evidence from literature on use of NIR in tablet manufacturing, measurement of dissolution from sustained release dosage form is an unexplored territory. Primary reason is that drug dissolution is a complex process dependent on many properties of the delivery system and dynamic changes occurring during the drug release. Drug dissolution rate depends on the type of dosage form (compressed/coated), solubility of the drug and interaction between drug and other excipients in tablet. NIR spectrum of a tablet contains sparse information about polymer characteristics, tablet hardness, water content, drug concentration etc. These properties can have a direct relationship with the rate of drug dissolution. However, the initial properties of tablets such as drug content, water content, hardness, density will change during the dissolution process. These changes are time and 
concentration dependent. Thus, the challenge in modeling drug dissolution with near infrared spectrum is the lack of complete information about dissolution process per se in the spectrum. However, if these changes are directly related to the initial properties of the system, then a valid relationship must exist between NIR spectrum and dissolution profile. This relationship may be linear or nonlinear depending on drug release kinetics. If the drug release is independent of time (Zero order), then initial concentration of the ingredients determine rate of drug release and the rate of drug release remains constant till the end of dissolution period. In such circumstances (zero order release), the relationship between NIR spectrum and drug release profile might be linear. If however, the drug release rate changes with time, then NIR spectrum-drug release profile relationship could be non-linear or may have no correlation.

Generally, drug release from sustained release matrix tablet is directly proportional to polymer concentration. However, polymer erosion, changes in drug concentration gradient, changes in drug diffusion path, changes in matrix integrity and tortousity can distort in the NIR spectrum-dissolution profile relationship, thus resulting in poor predictability of NIR models. Higuchi's square root time dependent release kinetics is the most common nonlinear drug release pattern observed in sustained release hydrophilic matrix tablets. In some cases, part of the drug dissolution profile is linear and the other part is nonlinear. That is, drug release rate remains constant in one part of the dissolution profile and release rate varies in the other part of the dissolution profile.

For modeling such complex relationships, multivariate regression models must estimate the spectral variability caused not only by the initial properties (hardness, thickness, moisture content, etc.) of the tablets, but also extract relevant information from the spectrum that has relationship with the drug dissolution from the tablets. For example, the initial drug release might 
be related to tablet hardness, however, in the later stages, as polymer hydrates, initial tablet hardness may not have any relationship with drug release. Since physical properties of the tablets and chemical nature of polymer influence the drug release interactively, it is difficult to attribute the changes to a specific peak in NIR spectrum. Rather, the spectral variance in a region can have relationship and this region can only be identified during calibration model building. Such modeling tasks are computationally intensive. Thus, selection of appropriate algorithm to complete the task quickly with no compromise on predictability of the model is important. This poster presents a comparison of multivariate modeling algorithms such as Partial Least Square, Support Vector Machines (SVM) and K-Nearest Neighbors (KNN). Partial Least Square (PLS) technique is well known in the pharmaceutical world and most commonly used NIR modeling technique. The other two techniques are uncommon in pharmaceutical industries, but have been used in food, agriculture, and business intelligence sectors. SVM and KNN are useful in special circumstances where non-linear, multivariate predictor-response relationship cannot be effectively modeled using PLS. This poster presents a comparison of prediction power of these three algorithms to estimate drug dissolution from sustained release matrix tablets containing propranolol hydrochloride.

\subsection{MATERIALS AND METHODS}

\subsubsection{Materials}

Propranolol hydrochloride, Carbopol 971P NF (Noveon Inc.,Cleveland, OH), Eudragit L 100 - 55 (Degussa Rohm Pharma Polymers, Piscataway, NJ), glyeryl monostearate (Stepan, Northfield, IL), Gelucire 50/13 (Gattefosse corporation, Paramus, NJ). 


\subsubsection{Methods}

\subsubsection{Granulation}

Hot melt granulation technique was used to prepare granules from the blend containing appropriate quantities of propranolol HCl, Carbopol 971P, Eudragit L-100-55, glyceryl monostearate (GMS) and Gelucire 50/13. A total of fifteen formulations were prepared by changing the composition of the aforementioned ingredients. Compositions of formulations are given in Table 5.1. The batch size of each formulation was $300 \mathrm{~g}$. A blend of waxy binder was prepared by melting GMS and Gelucire $50 / 13$ at $60^{\circ} \mathrm{C}$. The molten mass was mixed thoroughly and poured on a metal tray, and allowed to cool to room temperature. The resulting waxy sheet was cut into small size flakes, which were then used as a waxy binder for the hot melt granulation technique.

Ingredients of each formulation except the waxy binder were mixed for two minutes in the reverse mode at $1500 \mathrm{rpm}$ prior in a high shear mixer-granulator (Robot-Coupe ${ }^{\circledR}$ Inc., Jackson, MS). After blending, a circulating water bath was attached to the granulator bowl. Temperature of the bowl was monitored using a digital thermometer. Once the temperature of the bowl reached $60^{\circ} \mathrm{C}$, waxy binder was added to the powder blend. Bowl temperature was kept at $60^{\circ} \mathrm{C}$ during granulation. Granulation was carried out in the reverse mode at $1500 \mathrm{rpm}$ for 2 min. The resulting granules were passed through a 20 -mesh sieve to remove any coarse granules while they were still warm. The sieved granules were allowed to cool to room temperature. 
Table 5.1. Composition of formulations

\begin{tabular}{|c|c|c|c|c|c|}
\hline $\begin{array}{l}\text { Formulation } \dagger \\
\text { Code }\end{array}$ & $\begin{array}{c}\text { Glyceryl } \\
\text { monostearate } \\
(\%) \\
\end{array}$ & $\begin{array}{c}\text { Gelucire }{ }^{\circledR} \\
50 / 13 \\
(\%) \\
\end{array}$ & $\begin{array}{c}\text { Carbopol } \mathbb{R} \\
971 \mathrm{P} \\
(\%) \\
\end{array}$ & $\begin{array}{c}\text { Eudragit }{ }^{\circledR} \\
\text { L-100-55 } \\
(\%) \\
\end{array}$ & $\begin{array}{c}\text { Di-Tab } \mathbb{} \\
(\%)\end{array}$ \\
\hline PD-1 & 7.5 & 2.5 & 5 & 0 & 85 \\
\hline PD-2 & 7.5 & 2.5 & 5 & 20 & 65 \\
\hline PD-3 & 7.5 & 2.5 & 10 & 10 & 70 \\
\hline PD-4 & 7.5 & 2.5 & 15 & 0 & 75 \\
\hline PD-5 & 7.5 & 2.5 & 15 & 20 & 55 \\
\hline PD-6 & 5 & 5 & 5 & 10 & 75 \\
\hline PD-7 & 5 & 5 & 10 & 0 & 80 \\
\hline PD-8 & 5 & 5 & 10 & 10 & 70 \\
\hline PD-9 & 5 & 5 & 10 & 20 & 60 \\
\hline PD-10 & 5 & 5 & 15 & 10 & 65 \\
\hline PD-11 & 2.5 & 7.5 & 5 & 0 & 85 \\
\hline PD-12 & 2.5 & 7.5 & 5 & 20 & 65 \\
\hline PD-13 & 2.5 & 7.5 & 10 & 10 & 70 \\
\hline PD-14 & 2.5 & 7.5 & 15 & 0 & 75 \\
\hline PD-15 & 2.5 & 7.5 & 15 & 20 & 55 \\
\hline
\end{tabular}

$\uparrow 400 \mathrm{mg}$ tablets containing $80 \mathrm{mg}$ of propranolol hydrochloride were compressed at two compression forces $-0.6 \mathrm{mT}$ and $1.2 \mathrm{mT}$. 


\subsubsection{Tabletting}

Tablets weighing $400 \mathrm{mg}$ and containing $80 \mathrm{mg}$ of propranolol $\mathrm{HCl}$ were prepared by compressing the aforementioned granules. An 18-station rotary tablet press equipped with 3/8" flat-faced punches and dies was used for tablet compression. Each formulation was compressed at two compression forces, $0.6 \mathrm{mT}$ and $1.2 \mathrm{mT}$. A total of fifteen formulations, each compressed at two different compression forces yielded 30 batches of tablets. Three tablets from each batch were randomly selected for NIR measurements.

\subsubsection{NIR spectroscopy}

NIR spectrum (from $400 \mathrm{~nm}$ to $2500 \mathrm{~nm}$ ) of each tablet was taken from both sides using the Rapid Content Analyzer (Foss NIRSystems, Silver Spring, MD) in the reflectance mode. Constant spot size of light impingement of $9.25 \mathrm{~mm}$ was used for spectral measurements of all batches. Thirty two co-added scans were taken for each tablet to improve the signal-to-noise ratio, and they constitute one spectrum. Unprocessed NIR spectrum was exported to MicrosoftExcel $^{\circledR}$. The unprocessed spectrum for each tablet contained 4200 absorbance values, each taken at 0.5 nanometer gaps in the range of $400 \mathrm{~nm}$ to $2500 \mathrm{~nm}$.

\subsubsection{In vitro dissolution study}

Following NIR spectral measurement, each tablet was tested for its dissolution characteristics using USP apparatus II (paddle). Hydrochloric acid (0.1 N, $750 \mathrm{ml})$ was used as the dissolution media for the first 2 hours. Then, the $\mathrm{pH}$ of the dissolution media was adjusted to 6.8 using $250 \mathrm{ml}$ of $1 \mathrm{~N}$ tribasic phosphate solution. Drug dissolution from the tablets was determined at $37^{\circ} \mathrm{C}$ at $50 \mathrm{rpm}$. Drug release from the tablets was monitored using in situ fiber 
optic UV probes equipped with PDA (Photo diode array) detectors (PION, Inc., Woburn, MA). Estimation of the drug concentration in the dissolution media was based on single point calibration at $288 \mathrm{~nm}$. Drug dissolution profiles of all batches were calculated based on the UV absorbance, theoretical drug content, and volume of the dissolution media using Indigo ${ }^{\circledR}$ software (PION, Inc., Woburn, MA).

\subsubsection{Modeling}

Statistical models for correlating the NIR spectrum of 30 batches and their dissolution profiles were developed using STATISTICA ${ }^{\circledR} Q C \&$ Text miner (Statsoft, Tulsa, OK). Three regression modeling techniques such as PLS (Partial Least Square), SVM (support vector machine) and KNN (K-nearest neighbors) regression were used for screening purposes.

For selection of the best algorithm, regression coefficient of the observed versus predicted values were used. The best algorithm should have a high regression coefficient. The best algorithm was then used for building prediction models and validation. NIR spectral data from Formulation 13 were used as the validation set and hence they were not used in the while building the calibration model. Thus, calibration models were built using the remaining 14 formulations. These 14 formulations were randomly sampled and divided into 2 sets, namely training set (75\%), and test set (25\%). Calibration models were built and optimized using the Vfold cross validation protocol. In the V-fold cross-validation, repeated (v) random samples are drawn from the training and test sets, and the respective model is then applied to compute the predicted values. A regression coefficient of 0.90 for calibration models was used as the predefined selection criteria for algorithm selection. 


\subsection{RESULTS AND DISCUSSION}

\subsubsection{Algorithm screening}

\subsubsection{PLS modeling}

In conventional PLS modeling, calibration model is built to correlate spectral data with one output. However, dissolution profile is a repeated measure data, i.e., measurement of drug released from a single tablet over time. Owing to constraints of the PLS modeling, the entire dissolution profile cannot be modeled with one PLS model. Thus, for algorithm screening purposes, three calibration models were built to correlate the NIR spectrum and drug released at $2 \mathrm{~h}, 12 \mathrm{~h}$ and $24 \mathrm{~h}$ respectively. The models for predicting the amount of drug dissolved at $2 \mathrm{~h}$, $12 \mathrm{~h}$ and $24 \mathrm{~h}$ were named Y-2, Y-12 and Y-24 respectively. Regression coefficients of Y-2, Y12 and Y-24 were $0.839,0.853$ and 0.871 respectively indicating that only $83.9 \%, 85.3 \%$ and $87.1 \%$ of variance in the dissolution profiles were explained by these models. Y-2, Y-12 and Y24 models included 8, 5 and 8 latent vectors respectively. Number of latent vectors was selected based on the requirement of vectors required to explain $99.5 \%$ of variance observed in the data set.

Initially, selection of number of latent vector for global PLS model (model including entire spectrum) was based on cross-validation. Then, this number was optimized by screening different regions of spectum having maximum correlation with the response (amount of drug dissolved). This was done to improve the predictability of the model as this procedure removes noisy regions from model input. 
NIR spectrum as mentioned earlier is a multivariate spectrum. Spectral noise and irrelevant information (unrelated to the response) in the spectrum can reduce the predictability of the calibration model. These effects can be removed from the spectrum by scanning the spectrum in predefined windows (wave length ranges). For example, the entire spectrum in the range of 400 to $2500 \mathrm{~nm}$ (4200 absorbance readings) can be divided in to 20 windows each with 210 absorbance readings. Selection of window size depends on the complexity of the predictorresponse relationship. Complex relationships might demand multiple windows be combined for final prediction models. 20 windows is generally an optimum setting for screening as suggested by the literature. PLS models built using a region of wavelength of the entire spectrum (window) are local models, however, containing maximum information about the response of interest. Interestingly, $\mathrm{Y}-2, \mathrm{Y}-12, \mathrm{Y}-24$ prediction models had the following overlapping window regions: 2200 to $2394.5 \mathrm{~nm}$ for Y-2, 2185 to $2289.5 \mathrm{~nm}$ for Y-12, 2185 to $2289.5 \mathrm{~nm}$ for Y-24. In NIR spectrum, spectral region starting from $2200 \mathrm{~nm}$ has characteristic bands for $-\mathrm{CH},-\mathrm{CH}_{2}$ and $\mathrm{CH}_{3}$ groups. This could be attributed to polymers in the system. Release controlling polymers in the tablets used in this study were Carbopol and Eudragit. These two are acrylic acid derivatives. Carbopol is prepared by crosslinking acrylic acid monomer $\mathrm{CH}_{2} \mathrm{CH}(\mathrm{COOH})$ using allyl sucrose. Thus, spectral characteristics indicate a relationship between polymer content and drug release. However, the models did not have enough predictive power as judged by the pre-defined selection criteria. This could be attributed to limitations of the PLS modeling algorithm. PLS assumes linear relationship between predictors and response. As described earlier, linearity in the spectrum-dissolution profile can be distorted due to many factors. Thus, models that can account for nonlinearity in the spectrum-dissolution profile relationship could perform better. Hence, Support Vector Machine/Support Vector Regression algorithm was tested. 
For, literature provides evidence for model development using support vector machines where nonlinearity in predictor-response relationship had ill-influence on model predictability.

\subsubsection{SVM modeling}

\subsection{Theory}

Support vector machines (SVM) are based on concept of hyperplanes and decision boundaries. SVM algorithm maps the predictor variables and isolates them in hyper planes. For segregating (mapping) the predictor variables, decision boundaries are used in SVM. SVM was initially developed for classification tasks. In a simple classification task, assume that two objects with different qualities (e.g. blue and red) are present in a two dimensional plane (e.g. a flat surface). Geometrically defined place where the objects are present is called input space. To separate them, we must find the boundary line that can separate these two objects in the input space. This is analogous to drawing a line in a two dimensional plane to separate the blue and red objects. The process of drawing a decision line to separate objects in a hyper dimensional plane is called mapping. If a straight line is used for input mapping, then the line is called linear classifier. This forms the basis for statistical learning algorithms. This simple learning algorithm does not have enough power to explain multidimensional data (spectra data set) due to the data dimensionality, complexity, data density and colinearity characteristics. These difficult learning tasks are effectively handled by kernel learning algorithms such as SVM, multiple layer perceptron, etc.

In SVM, decision boundaries, instead of decision lines (used in linear regression), are used for input mapping. For classification task in a multidimensional space, it is ideal to select a 
hyper plane (decision line with boundaries) that correctly classifies the data, i.e., with a maximum distance to dissimilar objects. This distance between dissimilar objects is called a margin. Ideally, maximum margin must be achieved to facilitate accurate classification of a new data set. Thus, in a model training session, we must identify the hyperplane for separation of objects and maximizing the margin. Once identified and optimized, such hyperplanes are called maximum-margin hyperplanes or decision boundaries or optimal hyperplanes. Training set vectors that are close to the optimal hyperplanes are called support vectors. Optimal hyperplane provides probabilistic test error that is minimized when the margin is maximized.

Before mapping, predictors are spread in input space (multidimensional ill-defined hypothetical space). After mapping with decision boundaries, the SVM algorithm converts the input space into feature space (well defined space with less dimensions compared to input space). This process is known as dimensionality reduction. This is a common concept in Machine Learning Theory. Dimensionality reduction improves generalization and reduces computational burden.

In order to learn nonlinear predictor-response relationship using linear machines, the machine learning theory suggests usage of fixed non-linear mapping transforms that project data into a feature space. Then a linear machine is used to classify them in the feature space. However, such tasks are computationally intensive. Computational burden of such task can be reduced using kernels. Kernals are mathematical functions that transform data implicitly (in input space itself) into a feature space and train a linear machine in input space. Thus, concept of kernels is pivotal in machine learning algorithms, and SVM is one of the machine learning algorithms. Four types of kernels are commonly used in SVM regression: linear, polynomial, radial basis function and sigmoid. 
In SVM regression, a non-linear function is learned by a linear learning machine in a kernelinduced feature space, while the capacity of the system is controlled by a parameter that does not depend on the dimensionality of the space. In any regression technique, a relationship between predictors and responses is assumed and expressed by a function and noise term.

$$
y=f(x)+\text { noise }
$$

Now, the task is to find a functional form for $f$ that can correctly predict new cases that the SVM has not been presented with during training. This is achieved by training the SVM model using training set and optimizing the error function and decision boundaries. The functional form of predictor-response relationship in SVM regression includes kernels, slack variables and weight vectors. Kernels are used for feature mapping and slack variables are used for defining geometrical margins of decision boundaries to improve generalization. Slack variables are the measure of error on training data points. Hence, selection of appropriate kernel and optimization of training parameters yield functional form of predictor-response relationship. Parameters in the functions are optimized using cross-validation during model training. Cross validation prevents "over learning" of the model. "Over learning" is the process by which the accuracy of trend identification is hampered by error present in the data. "Over learning" significantly affects the predictability of the model. Model may continuously over predict or under predict when "over learning" happens during model building. "Over learning" can be prevented using cross validation during training process. 


\subsection{Results}

SVM models for Y-2, Y-12, and Y-24 were developed with linear, polynomial and radial basis function kernels. Degree of polynomials was optimized for each model. Polynomial degree of 2 was optimal for the Y-2, Y-12 and Y-24 models as increase in polynomial degree did not provide any improvement in regression coefficients. Regression coefficients for Y-2 models with linear, polynomial and RBF kernels were $0.814,0.710$ and 0.773 respectively. Regression coefficients for Y-12 models with linear, polynomial and RBF kernels were $0.805,0.704$ and 0.824 respectively. Regression coefficients for Y-24 models were $0.790,0.659$ and 0.783 . It can be inferred that SVM based on linear and RBF kernels had better explanatory power than models with polynomial kernel. However, the regression coefficients of models with linear and RBF kernels were lower than that of the PLS models. This implies that in spite of their sophisticated kernel based input mapping principle, SVM regression failed to offer better predictability than the PLS models. This could be attributed to predictor variance distribution. PLS model had specific predictor regions (windows) as model input. This region had maximum correlation with out puts; hence prediction errors caused by noisy inputs were eliminated. However, SVM uses the entire spectrum for modeling. Owing to "Garbage in-Garbage out" modeling principle, when noisy variables having little or no correlation with outputs are used as predictors, the model predictability is less. Thus predictability can be improved by selecting a spectral region that has maximum correlation with the cumulative amount of drug release. In addition, default training parameters were used for model training in this study. If the training parameters are optimized for each kernel type, then predictability could improve. Based on better predictability of linear kernels, it can be inferred that the predictors have valid linear relationship with responses. This means, the drug release (response) has a linear relationship with variance observed in the NIR 
spectrum (predictor). If the variance in the NIR spectrum is directly related to the concentration of the polymers and excipients, then the drug release is directly related to polymer and excipient concentration.

\subsubsection{KNN modeling}

\subsection{Theory}

K-Nearest neighbor (KNN) models are based on combination of local models. This means that the statistical analysis for the whole data set is split into local analyses and then combined. Predictors and responses of the training set are sampled and distance function is introduced between predictors. This distance function defines each predictor value. Then a neighborhood is formed by the predictors that are close to one another in terms of the distance between predictor values. In KNN, response from an unknown predictor is calculated by using the group of predictor values (neighborhood) that are close to the unknown predictor value. Thus predictability of the KNN model depends on the K value that defines number of elements in each neighborhood. Once the neighborhood of the unknown predictor value is identified, the predicted response will be the arithmetic mean response value of responses of predictor values in the neighborhood. KNN model fits data closely which might lead to overfitting, i.e., poor predictability caused by lack of generalization of the calibration model. Thus, distance function and cardinality of the neighborhood $(K)$ must be carefully selected for building models with better predictability. $\mathrm{K}$ value is an indicator of adoptability of the model. Higher the $\mathrm{K}$ value, poorer the predictability. If the $\mathrm{K}$ value is small, then the model lacks generalization power to predict new cases. Optimal K value can be selected using V-fold cross-validation. In V-fold 
cross-validation, the number of folds (subsets of training data) is defined. For example, if the data is set to be divided into 10 folds, 10 subsets are created using random sampling. Then, 9 subsets (number of folds minus one) will be used for training, and the unused set serves as the internal validation set. Prediction error of the model developed using 9 subsets, for the internal validation set is monitored to stop the training process. In the beginning of the training, the prediction error decreases as an appropriate $\mathrm{K}$ value is achieved. Then, if the $\mathrm{K}$ value continues to increase, the prediction error also starts to increase, thus indicating data overfitting. The training is terminated when the prediction error starts to increase, and the $\mathrm{K}$ value required for least prediction error is selected as the optimal $\mathrm{K}$ value. This process is repeated by swapping the internal validation set from one training subset. Thus, in the second iteration, a new internal validation serves to prevent overfitting. Once all the training subsets have served as internal validation sets, the training error is calculated by averaging errors observed in each iteration.

\subsection{Results}

KNN models were developed using four different types of distance measures, namely Euclidean, Euclidean squared, Cityblock and Chebychev. Numbers of nearest neighbors were optimized using V-fold cross-validation for each distance measure. Cityblock distance measure and three nearest neighbors were identified as the best distance training settings in terms of predictability. Regression coefficients for Y-2, Y-12 and Y-24 models were 0.972, 0.964 and 0.979. These regression values satisfied pre-set selection criteria of 0.9 for prediction models. Thus, KNN algorithm was used to model the drug dissolution profile, i.e., amount of drug released at 1 h, 2 h, 4 h, 6 h, 8 h, 10 h, 12 h, 14 h, 16 h, 18 h, 20 h, 22 h and 24 h. The KNN 
algorithm can handle multiple outputs. Thus, only one model was with Cityblock distance measure and three nearest neighbors was developed.

\subsubsection{Model validation}

KNN model was developed for predicting the entire dissolution profile using Cityblock distance measure. Observed and predicted values for the dissolution data obtained from Formulation 13 for both low and high compression forces are given in Figure 5.1. and Figure 5.2, respectively. The regression coefficients between observed and predicted values were 0.984 and 0.981 for formulation 13 prepared at low and high compression forces respectively.

\subsection{CONCLUSION}

Dissolution profiles of propranolol hydrochloride from sustained release matrix tablets were predicted from NIR spectra of tablets. Selection of algorithm was important to achieve the best predictability. K-nearest neighbor algorithm had better predictability than partial least square and support vector machine algorithms. 


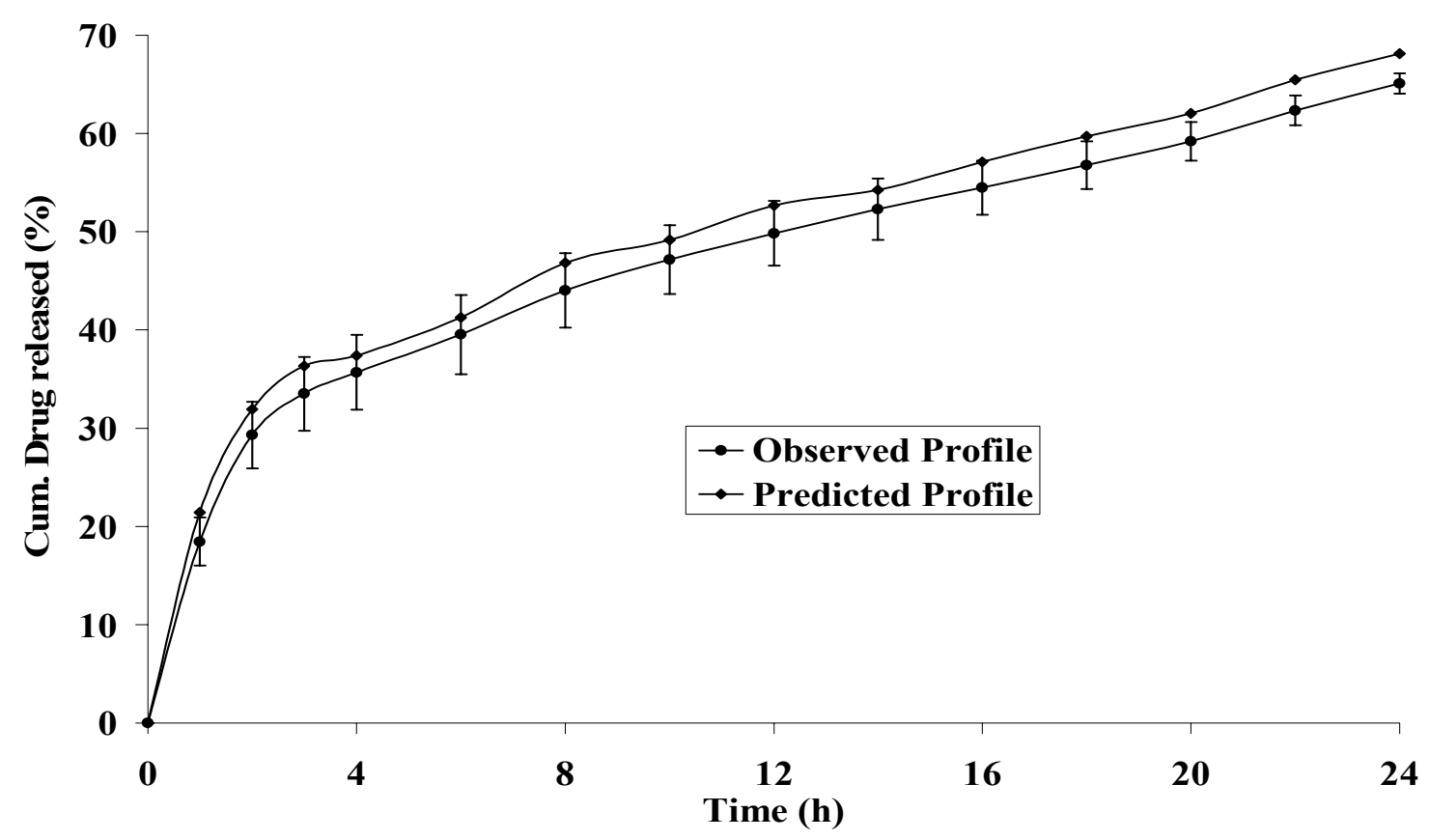

Figure 5.1. Validation using formulation 13 prepared at $0.6 \mathrm{mT} \dagger$

$\dagger$ Each data point represents average of three measurements. Standard deviation of three measurements is presented as error bars. 


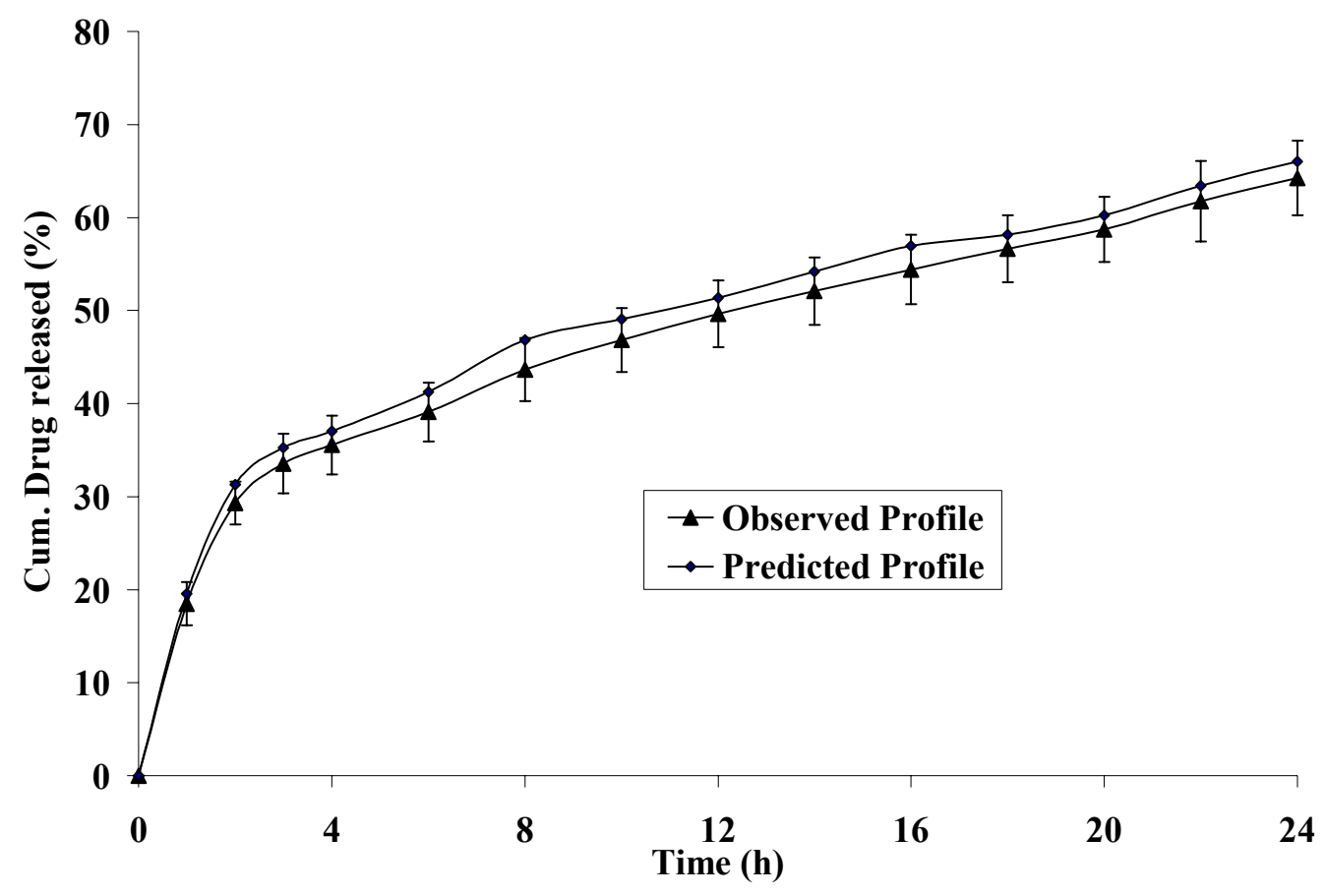

Figure 5.2. Validation using formulation 13 prepared at $1.2 \mathrm{mT}$

$\uparrow$ Each data point represents average of three measurements. Standard deviation of three measurements is presented as error bars. 


\section{LIST OF REFERENCES}

1. Higuchi $\mathrm{T}$, Rate for release of medicaments from ointment bases containing drugs in suspensions. J Pharm Sci, 1961. 50(3): p. 874-875.

2. Peppas NA, Analysis of fickian and non-fickian drug release from polymers. Pharm Acta Helv, 1985. 60(5): p. 110-111.

3. Peppas NA, Korsmeyer RW, Dynamically swelling hydrogels in controlled release applications. Hydrogens in medicine and pharmacy. Vol. 3. 1986, Boca Raton: CRC Press. 109-136.

4. $\quad$ Wang TT, Kwei TK, Frisch HL, Diffusion in glassy polymers iii. J Polym Sci, 1968. 7(4): p. 2019-2028.

5. Park GS, Transport principles - solution, diffusion and permeation in polymer membranes. Synthetic membranes: Science, engineering and applications, ed. Bungay PM, Lonsdale, M.N. Vol. 1. 1986, Dordrecht: D. Reidel Publishing Company. p. 57-108.

6. $\quad$ Frisch HL, Sorption and transport in glassy polymers. Polym Eng Sci, 1980. 20(4): p. 213.

7. Ritger PL, Peppas NA, A simple equation for description of solute release. I. Fickian and non-fickian release from non-swellable devices in the form of slabs, spheres, cylinders or discs. J Control Release, 1987. 5(2): p. 23-26.

8. Ritger PL, Peppas NA, A simple equation for description of solute release ii. Fickian and anomalous release from swellable devices. J Control Release, 1987. 5(2): p. 37-42.

9. Peppas NA, Sahlin JJ, A simple equation for the description of solute release. Iii. Coupling of diffusion and relaxation. Int J Pharm, 1989. 57(6): p. 169-172.

10. Noveon, Controlled release formulations using carbopol polymers, in Carbopol Pharmaceutical applications. 1999, Noveon Inc.: Cleveland. p. 1-59.

11. Khan GM, Zhu JB, Studies on drug release kinetics from ibuprofen-carbomer hydrophilic matrix tablets: Influence of co-excipients on release rate of the drug. $\mathrm{J}$ Control Release, 1999. 57(2): p. 197-203.

12. Khan GM, Jiabi Z, Formulation and in vitro evaluation of ibuprofen-carbopol $974 p-n f$ controlled release matrix tablets. Iii: Influence of co-excipients on release rate of the drug. J Control Release, 1998. 54(2): p. 185-190. 
13. Salib NN, El-Gamal SA, Application of some polymers in the physiocochemical design of tablet formulation. Pharmazie, 1976. 31(10): p. 718-721.

14. Baun DC, Walker GC, The prolonged release of caramiphen hydrochloride and atropine sulfate from compressed tablets containing carbopol 934. Pharm Acta Helv, 1971. 46(2): p. 94-113.

15. Bravo SA, Lamas MC, Salomon CJ, Swellable matrices for the controlled-release of diclofenac sodium: Formulation and in vitro studies. Pharm Dev Technol, 2004. 9(1): p. 75-83.

16. Jelena P, Djuric Z, Jovanovic M, Ibric S, Kilibarda V, Jovanovic D, Evic IK, Biopharmaceutical characterization of sustained release matrix tablets based on novel carbomer polymers: Formulation and in vivo investigation. Eur J Drug Metab Pharmacokinet, 2005. 30(1-2): p. 99-104.

17. Llabres M, Farina JB, Design and evaluation of sustained-release tablets of lithium in a fat matrix and its bioavailability in humans. J Pharm Sci, 1991. 80(11): p. 1012-1016.

18. Genc L, Bilac H, Guler E, Studies on controlled release dimenhydrinate from matrix tablet formulations. Pharm Acta Helv, 1999. 74(1): p. 43-49.

19. Rao YM, Veni JK, Jayasagar G, Formulation and evaluation of diclofenac sodium using hydrophilic matrices. Drug Dev Ind Pharm, 2001. 27(8): p. 759-766.

20. Emami J, Tavakoli N, Movahedian A, Formulation of sustained - release lithium carbonate matrix tablets: Influence of hydrophilic materials on the release rate and in vitro-in vivo evaluation. J Pharm Pharm Sci, 2004. 7(3): p. 338-344.

21. El-Malah Y, Nazzal S, Hydrophilic matrices: Application of placket-burman screening design to model the effect of polyox-carbopol blends on drug release. Int J Pharm, 2006. 309(1-2): p. 163-170.

22. El-Malah Y, Nazzal S, Khanfar NM, D-optimal mixture design: Optimization of ternary matrix blends for controlled zero-order drug release from oral dosage forms. Drug Dev Ind Pharm, 2006. 32(10): p. 1207-1218.

23. Florence A, Jani P, Novel oral - drug formulations - their potential in modulating adverse - effects. Drug Safety, 1994. 410(3): p. 233-266.

24. Baun DC, George W, Prolonged release of caramiphen hydrochloride and atropine sulfate from compressed tablets containing carbopol 934. Pharm Acta Helv, 1994. 46(2): p. $94-113$.

25. Choulis NH, Papadopoulos H., Choulis, M., Long acting methadone. Pharmazie, 1976. 31(H): p. 7-13. 
26. Durrani MJ, Whitaker R, Benner SC, A comparative study of controlled release agents. I. Effects of compression force and polymer concentration. Amer Assoc Pharm Sci, 1992. 9(2): p. 23-31.

27. Durrani MJ, Whitaker R, Andrews A, Greenberg E, Wiggins M, Studies on drug release kinetics from carbopol 974p tablets. in Sixth AAPS annual meeting. 1991. Washington D.C.: American Association of Pharmaceutical Scientists.

28. Durrani MJ, Todd R, Andrews A, Whitaker RW, Greenberg E., Benner SC, Polymer concentration effects on carbopol 934 p tablets for controlled release. in Proc Int Symp Controlled Release Bioact Mater. 1992. Controlled Release Society, London.: p. 132134.

29. Durrani MJ, Manji PA, Whitaker RF, Huvard GS, Controlled-release studies on carbopol 971p polymer. in Proc Int Symp Controlled Release Bioact Mater. 1994. Controlled Release Society, Austria.: p. 146-148.

30. Perez-Marcos B, Gutierrez C, Gomez-Amoza,JL, Martinez-Pacheco R, Souto C, Concheiro A., Usefulness of certain varieties of carbomer in the formulation of hydrophilic furosemide matrices. International Journal of Pharmaceutics, 1991. 67(2): p. 113-121.

31. Perez Marcos B, Iglesias R, Gomez-Amoxa JL, Mechanical and drug release properties of atenolol-carbomer hydrophillic matrix tablets. J Controlled Release, 1991. 17(3): p. 267-276.

32. Cooper AM, Doshi DH, Patel MV, In vitro prolonged release of basic cns compounds from hpmc and carbopol 974p hydrophillic matrixes: Polymer/drug ionization and counterion effects. in Proc Int Symp Controlled Release Bioact Mater. 1995. Controlled Release Society, Minneapolis.: p. 352-354.

33. Huang L, Schwartz J, Studies on drug release from a carbomer tablet matrix. Drug Dev Ind Pharm, 1995. 21(13): p. 1487-1501.

34. Bulet-Oner F, Sustained release isoniazid tablets $i$. Formulation and in vitro evaluation $\mathrm{J}$ Pharm Sci, 1989. 59(4): p. 678-685.

35. Govender T, Ehtezazi T, Stolnik S, Illum L, Davis SS, Complex formation between the anionic polymer (paa) and a cationic drug (procaine hci): Characterization by microcalorimetric studies. Pharm Res, 1999. 16(7): p. 1125-1131.

36. Tatavarti AS, Mehta KA, Augsburger LL, Hoag SW, Influence of methacrylic and acrylic acid polymers on the release performance of weakly basic drugs from sustained release hydrophilic matrices. J Pharm Sci, 2004. 93(9): p. 2319-2331. 
37. Blanco-Fuente H, Esteban-Fernandez B, Blanco-Mendez J, Otero-Espinar FJ, Use of beta-cyclodextrins to prevent modifications of the properties of carbopol hydrogels due to carbopol-drug interactions. Chem Pharm Bull, Tokyo, 2002. 50(1): p. 40-46.

38. Lehr CM, Bouwstra JA, Tukker JJ, Verhoef AC, De Boer AG, Junginger HE, Breimer $\mathrm{DD}$, Oral bioadhesive drug delivery systems-effect on g.I. Transit and peptide absorption. Pharm Res, 1990. 7(9): p. 722-726.

39. Chang H, Park H, Kelly P, Robinson J, Bioadhesive polymers as platforms for oral controlled release drug delivery. Ii. Synthesis and evaluation of some swelling, waterinsoluble bioadhesive polymers. J Pharm Sci, 1985. 74(4): p. 399-405.

40. Agarwal V, Mishra B, Design, development, and biopharmaceutical properties of buccoadhesive compacts of pentazocine. Drug Dev Ind Pharm, 1999. 25(6): p. 701-709.

41. Ahuja A, Khar RK, Chaudhry R, Evaluation of buccoadhesive metronidazole tablets: Microbiological response. Pharmazie, 1998. 53(4): p. 264-267.

42. Ali J, Khar RK, Ahuja A, Formulation and characterisation of a buccoadhesive erodible tablet for the treatment of oral lesions. Pharmazie, 1998. 53(5): p. 329-334.

43. Ameye D, Honraet K, Loose D, Vermeersch H, Nelis H, Remon JP, Effect of a buccal bioadhesive nystatin tablet on the lifetime of a provox silicone tracheoesophageal voice prosthesis. Acta Otolaryngol, 2005. 125(3): p. 304-306.

44. Ameye D, Mus D, Foreman P, Remon JP, Spray-dried amioca starch/carbopol 974p mixtures as buccal bioadhesive carriers. Int J Pharm, 2005. 301(1-2): p. 170-180.

45. Anlar S, Capan Y, Guven O, Gogus A, Dalkara T, Hincal AA, Formulation and in vitroin vivo evaluation of buccoadhesive morphine sulfate tablets. Pharm Res, 1994. 11(2): $\mathrm{p}$. 231-236.

46. Attama AA,Adikwu MU, Melt extrusion bioadhesive delivery of diclofenac sodium granules using theobroma oil. Boll Chim Farm, 2004. 143(4): p. 174-177.

47. Attama AA, Adikwu MU, Amorha CJ, Release of indomethacin from bioadhesive tablets containing carbopol 941 modified with abelmuschus esculentus (okra) gum. Boll Chim Farm, 2003. 142(7): p. 298-302.

48. Baloglu E, Ozyazici M, Hizarcioglu SY, Karavana HA, An in vitro investigation for vaginal bioadhesive formulations: Bioadhesive properties and swelling states of polymer mixtures. Farmaco, 2003. 58(5): p. 391-396.

49. Baloglu E, Ozyazici M, Yaprak Hizarcioglu S, Senyigit T, Ozyurt D, Pekcetin C, Bioadhesive controlled release systems of ornidazole for vaginal delivery. Pharm Dev Tech, 2006. 11(4): p. 477-484. 
50. Betageri GV, Deshmukh DV, Gupta RB, Oral sustained-release bioadhesive tablet formulation of didanosine. Drug Dev Ind Pharm, 2001. 27(2): p. 129-136.

51. Bouckaert S, Lefebvre RA, Remon JP, In vitro/in vivo correlation of the bioadhesive properties of a buccal bioadhesive miconazole slow-release tablet. Pharm Res, 1993. 10(6): p. 853-856.

52. Bouckaert S, Remon JP, In-vitro bioadhesion of a buccal, miconazole slow-release tablet. J Pharm Pharmacol, 1993. 45(6): p. 504-507.

53. Elkheshen S, Yassin AE, Alkhaled F, Per-oral extended-release bioadhesive tablet formulation of verapamil hcl. Boll Chem Farm, 2003. 142(5): p. 226-231.

54. El-Samaligy MS, Yahia SA, Basalious EB, Formulation and evaluation of diclofenac sodium buccoadhesive discs. Int J Pharm, 2004. 286(1-2): p. 27-39.

55. Gai MN, Isla A, Andonaegui MT, Thielemann AM, Seitz C, Evaluation of the effect of 3 different diets on the bioavailability of 2 sustained release theophylline matrix tablets. Int J Clin Pharmacol Ther, 1997. 35(12): p. 565-571.

56. Hosny EA, Elkheshen SA, Saleh SI, Buccoadhesive tablets for insulin delivery: In-vitro and in-vivo studies. Boll Chem Farm, 2002. 141(3): p. 210-217.

57. Ikinci G, Senel S, Wilson CG, Sumnu M, Development of a buccal bioadhesive nicotine tablet formulation for smoking cessation. Int J Pharm, 2004. 277(1-2): p. 173-178.

58. Jug M, Becirevic-Lacan M, Influence of hydroxypropyl-beta-cyclodextrin complexation on piroxicam release from buccoadhesive tablets. Eur J Pharm Sci, 2004. 21(2-3): p. 251260.

59. Minghetti P, Pacchetti B, Montanari L, Ronchi C, Berlati F, Buccoadhesive tablets for the slow delivery of cetylpyridinium chloride: Design and in vitro/in vivo analysis. Boll Chem Farm, 1997. 136(7): p. 543-548.

60. Mohammed FA, Khedr H, Preparation and in vitro/in vivo evaluation of the buccal bioadhesive properties of slow-release tablets containing miconazole nitrate. Drug Dev Ind Pharm, 2003. 29(3): p. 321-337.

61. Narendra C, Srinath MS, Prakash Rao B, Development of three layered buccal compact containing metoprolol tartrate by statistical optimization technique. Int J Pharm, 2005. 304(1-2): p. 102-114.

62. Nur AO, Zhang JS, Captopril floating and/or bioadhesive tablets: Design and release kinetics. Drug Dev Ind Pharm, 2000. 26(9): p. 965-969. 
63. Owens TS, Dansereau RJ, Sakr A, Development and evaluation of extended release bioadhesive sodium fluoride tablets. Int J Pharm, 2005. 288(1): p. 109-122.

64. Weyenberg W, Bozdag S, Foreman P, Remon JP, Ludwig A, Characterization and in vivo evaluation of ocular minitablets prepared with different bioadhesive carbopol-starch components. Eur J Pharm Biopharm, 2006. 62(2): p. 202-209.

65. Singh B,Ahuja N, Development of controlled-release buccoadhesive hydrophilic matrices of diltiazem hydrochloride: Optimization of bioadhesion, dissolution, and diffusion parameters. Drug Dev Ind Pharm, 2002. 28(4): p. 431-442.

66. Weyenberg W, Vermeire A, Vandervoort J, Remon JP, Ludwig A, Effects of roller compaction settings on the preparation of bioadhesive granules and ocular minitablets. Eur J Pharm Biopharm, 2005. 59(3): p. 527-536.

67. Satoh K, Takayama K, Machida Y, Suzuki Y, Nakagaki M, Nagai T, Factors affecting the bioadhesive property of tablets consisting of hydroxypropyl cellulose and carboxyvinyl polymer. Chem Pharm Bull (Tokyo), 1989. 37(5): p. 1366-1368.

68. Varshosaz J, Tavakoli N, Roozbahani F, Formulation and in vitro characterization of ciprofloxacin floating and bioadhesive extended-release tablets. Drug Deliv, 2006. 13(4): p. 277-285.

69. Singh B, Chakkal SK, Ahuja N, Formulation and optimization of controlled release mucoadhesive tablets of atenolol using response surface methodology. AAPS PharmSciTech, 2006. 7(1): p. E3.

70. Leucuta SE, Ponchel G, Duchene D, Oxprenolol release from bioadhesive gelatin/poly(acrylic acid) microspheres. J Microencapsul, 1997. 14(4): p. 511-522.

71. Geraghty PB, Attwood D, Collett JH, Sharma H, Dandiker Y, An investigation of the parameters influencing the bioadhesive properties of myverol 18-99/water gels. Biomaterials, 1997. 18(1): p. 63-67.

72. Capan Y, Senel S, Calis, S, Takka, S, Hincal, AA, Formulation and in vitro-in vivo evaluations of sustained release acetylsalicylic acid tablets. Pharmaceutical Industry, 1989. 51(4): p. 443-448.

73. Ciftci K, Capan, Y, Ozturk, O, Hincal, A, Formulation and in vitro-in vivo evaluation of sustained release lithium carbonate tablets. Pharm Res, 1990. 7(4): p. 359-363.

74. Celebi N, Kislal O, Development and evaluation of a buccoadhesive propranolol tablet formulation. Pharmazie, 1995. 50(7): p. 470-472.

75. Ceschel GC, Maffei P, Lombardi Borgia S, Ronchi C, Design and evaluation of buccal adhesive hydrocortisone acetate (hca) tablets. Drug Deliv, 2001. 8(3): p. 161-171. 
76. Park CR, Munday DL, Development and evaluation of a biphasic buccal adhesive tablet for nicotine replacement therapy. Int J Pharm, 2002. 237(1-2): p. 215-226.

77. Du Q, Ping QN, Liu GJ, Preparation of buspirone hydrochloride buccal adhesive tablet and study on its drug release mechanism. Yao Xue Xue Bao, 2002. 37(8): p. 653-656.

78. Perioli L, Ambrogi V, Rubini D, Giovagnoli S, Ricci M, Blasi P, Rossi C, Novel mucoadhesive buccal formulation containing metronidazole for the treatment of periodontal disease. J Control Release, 2004. 95(3): p. 521-533.

79. Varshosaz J, Dehghan Z, Development and characterization of buccoadhesive nifedipine tablets. Eur J Pharm Biopharm, 2002. 54(2): p. 135-141.

80. Desai KG, Kumar TM, Preparation and evaluation of a novel buccal adhesive system. AAPS PharmSciTech, 2004. 5(3): p. e35.

81. Mohammadi-Samani S, Bahri-Najafi R, Yousefi G, Formulation and in vitro evaluation of prednisolone buccoadhesive tablets. Farmaco, 2005. 60(4): p. 339-344.

82. Gavini E, Sanna V, Juliano C, Bonferoni MC, Giunchedi P, Mucoadhesive vaginal tablets as veterinary delivery system for the controlled release of an antimicrobial drug, acriflavine. AAPS PharmSciTech, 2002. 3(3): p. E20.

83. Valenta C, Kast CE, Harich I, Bernkop-Schnurch A, Development and in vitro evaluation of a mucoadhesive vaginal delivery system for progesterone. J Control Release, 2001. 77(3): p. 323-332.

84. Chopra S, Motwani SK, Iqbal Z, Talegaonkar S, Ahmad FJ, Khar RK, Optimisation of polyherbal gels for vaginal drug delivery by box-behnken statistical design. Eur J Pharm Biopharm, 2006. 66(1): p. 73-82.

85. Ceulemans J, Vermeire A, Adriaens E, Remon JP, Ludwig A, Evaluation of a mucoadhesive tablet for ocular use. J Control Release, 2001. 77(3): p. 333-344.

86. Weyenberg W, Vermeire A, Remon JP, Ludwig A, Development and evaluation of sustained release ocular minitablets. J Control Release, 2005. 101(1-3): p. 371-374.

87. Jimenez-Kairuz A, Allemandi D, Manzo RH, Mechanism of lidocaine release from carbomer-lidocaine hydrogels. J Pharm Sci, 2002. 91(1): p. 267-272.

88. Jimenez-Kairuz AF, Allemandi DA, Manzo RH, Equilibrium properties and mechanism of kinetic release of metoclopramide from carbomer hydrogels. Int J Pharm, 2003. 250(1): p. 129-136. 
89. Liu DZ, Sheu MT, Chen CH, Yang YR, Ho HO, Release characteristics of lidocaine from local implant of polyanionic and polycationic hydrogels. J Control Release, 2007. 118(3): p. 333-339.

90. Mura P, Bettinetti GP, Liguori A, Bramanti G, Improvement of clonazepam release from a carbopol hydrogel. Pharm Acta Helv, 1992. 67(9-10): p. 282-288.

91. Traue J, Kala H, Use of polyacrylate dispersions for nebulization of phenobarbital. 2. In vitro testing of tablets with phenobarbital polyacrylate microspheres. Pharmazie, 1984. 39(5): p. 331-333.

92. Glavas-Dodov M, Goracinova K, Mladenovska K, Fredro-Kumbaradzi E, Release profile of lidocaine hcl from topical liposomal gel formulation. Int J Pharm, 2002. 242(1-2): p. 381-384.

93. Okore VC, Effect of dika fat content of a barrier film coating on the kinetics of drug release from swelling polymeric systems. Boll Chim Farm, 2000. 139(1): p. 21-25.

94. Bhardwaj SB, Directly compressible polymer matrix tablets, in Pharmaceutics. 1989, Duquesne University: Philadelphia. p. 89.

95. Capan Y, Ciftci, K., Hincal, A., Influence of filler excipients on the release rate of sustained release lithium carbonate tablets. Eur J Pharm Biopharm, 1991. 37(1): p. 1418.

96. Barreiro-Iglesias R, Bromberg L, Temchenko M, Hatton TA, Alvarez-Lorenzo C, Concheiro A, Pluronic-g-poly(acrylic acid) copolymers as novel excipients for site specific, sustained release tablets. Eur J Pharm Sci, 2005. 26(5): p. 374-385.

97. Cilurzo F, Minghetti P, Selmin F, Casiraghi A, Montanari L, Polymethacrylate salts as new low-swellable mucoadhesive materials. J Control Release, 2003. 88(1): p. 43-53.

98. Pringels E, Ameye D, Vervaet C, Foreman P, Remon JP, Starch/carbopol spray-dried mixtures as excipients for oral sustained drug delivery. J Control Release, 2005. 103(3): p. 635-641.

99. Chow M, Fabrication and characterization of extruded and spheronized pellets containing carbopol 974p. Pharm Res, 1990. 11(10): p. 21-28.

100. Young CR, Dietzsch C, Cerea M, Farrell T, Fegely KA, Rajabi-Siahboomi A, McGinity JW, Physicochemical characterization and mechanisms of release of theophylline from melt-extruded dosage forms based on a methacrylic acid copolymer. Int J Pharm, 2005. 301(1-2): p. 112-120. 
101. Zhu Y, Shah NH, Malick AW, Infeld MH, McGinity JW, Influence of thermal processing on the properties of chlorpheniramine maleate tablets containing an acrylic polymer. Pharm Dev Technol, 2002. 7(4): p. 481-489. 


\section{VITA}

Natarajansoundarapandian Mariageraldrajan was born on May 21, 1975 in Madurai,

India. He received his degree in Bachelor of Pharmacy from the Dr. M. G. R. Medical

University, India in April 1996. He joined the graduate school at The University of Tennessee

Health Science Center, Memphis in the fall of 2002. 